\title{
Aeroelastic Analysis of a Turbine Blade Using a Reduced-Order Model
}

\author{
by \\ Aimée Haché \\ B.Eng. (Mechanical) \\ A thesis submitted to \\ the Faculty of Graduate Studies and Research \\ in partial fulfilment of \\ the requirements for the degree of \\ Master of Applied Science \\ Ottawa-Carleton Institute for \\ Mechanical and Aerospace Engineering \\ Department of Mechanical and Aerospace Engineering \\ Carleton University \\ Ottawa, Ontario, Canada \\ January 2007 \\ Copyright (C) \\ 2007 - Aimée Haché
}




$\begin{array}{ll}\begin{array}{l}\text { Library and } \\ \text { Archives Canada }\end{array} & \begin{array}{l}\text { Bibliothèque et } \\ \text { Archives Canada }\end{array} \\ \begin{array}{l}\text { Published Heritage } \\ \text { Branch }\end{array} & \begin{array}{l}\text { Direction du } \\ \text { Patrimoine de l'édition }\end{array} \\ \begin{array}{l}\text { 395 Wellington Street } \\ \text { Ottawa ON K1A ON4 }\end{array} & \begin{array}{l}\text { 395, rue Wellington } \\ \text { Ottawa ON K1A ON4 } \\ \text { Canada }\end{array}\end{array}$

Your file Votre référence ISBN: 978-0-494-26990-9 Our file Notre référence ISBN: 978-0-494-26990-9

NOTICE:

The author has granted a nonexclusive license allowing Library and Archives Canada to reproduce, publish, archive, preserve, conserve, communicate to the public by telecommunication or on the Internet, loan, distribute and sell theses worldwide, for commercial or noncommercial purposes, in microform, paper, electronic and/or any other formats.

The author retains copyright ownership and moral rights in this thesis. Neither the thesis nor substantial extracts from it may be printed or otherwise reproduced without the author's permission.
AVIS:

L'auteur a accordé une licence non exclusive permettant à la Bibliothèque et Archives Canada de reproduire, publier, archiver, sauvegarder, conserver, transmettre au public par télécommunication ou par l'Internet, prêter, distribuer et vendre des thèses partout dans le monde, à des fins commerciales ou autres, sur support microforme, papier, électronique et/ou autres formats.

L'auteur conserve la propriété du droit d'auteur et des droits moraux qui protège cette thèse. $\mathrm{Ni}$ la thèse ni des extraits substantiels de celle-ci ne doivent être imprimés ou autrement reproduits sans son autorisation.
In compliance with the Canadian

Privacy Act some supporting forms may have been removed from this thesis.

While these forms may be included in the document page count, their removal does not represent any loss of content from the thesis.
Conformément à la loi canadienne sur la protection de la vie privée, quelques formulaires secondaires ont été enlevés de cette thèse.

Bien que ces formulaires aient inclus dans la pagination, il n'y aura aucun contenu manquant.

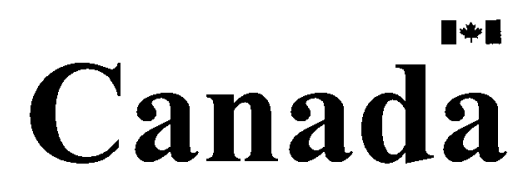




\section{Abstract}

The following work examines a turbine blade as a rotating cantilever beam fixed to a rigid hub. The first three natural mode shapes of the structure were determined using ANSYS-10.0. Unsteady computational fluid dynamic analysis was conducted to achieve the fluid response due to the natural modes of vibration of the rotating blade. A reduced-order model was used to couple the structural and aerodynamic system data. This resulted in an eigenfunction in the determination of a transfer function whose poles define the stability of the aeroelastic system. The same transfer

function can be used to study problems of aeroelastic response of a turbine blade to arbitrary inputs such as due to structural damage or unbalanced mass. 
This work is dedicated to my parents who have always believed that I could accomplish anything. 


\section{Acknowledgments}

I would like to acknowledege my thesis supervisors, Dr. F. Nitzsche and Dr. J.E.D. Gauthier, for their continued support, both financially and intellectually. Much thanks to Dr. M. Benner from the National Research Council of Canada, without whom this project would never have come to me. I also acknowledge Dr. E. Matida for his eager help with the CFD simulations. And last, but certainly not least, Dr. M. Ghorashi, who was instrumental in the development of the structural field equations. 


\section{Table of Contents}

$\begin{array}{ll}\text { Abstract } & \text { ii }\end{array}$

Acknowledgments $\quad$ iv

Table of Contents $\quad$ v

List of Tables viii

List of Figures $\quad$ ix

Nomenclature $\quad$ xi

1 INTRODUCTION 1

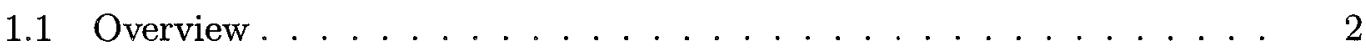

1.2 Literature Review . . . . . . . . . . . . . . . . . . 3

2 NATURAL MODE SHAPE CALCULATION USING ANSYS-10.0 7

3 TRANSIENT AERODYNAMIC FORCE DETERMINATION US$\begin{array}{ll}\text { ING CFX-10.0 } & 17\end{array}$

3.1 Fluid Domain . . . . . . . . . . . . . . . . . . . . . 18

3.2 Mesh Creation . . . . . . . . . . . . . . . . . . . 19

3.2.1 Structured Grid . . . . . . . . . . . . . . . . 20

3.2 .2 Unstructured Grid . . . . . . . . . . . . . . . . . 20 
3.3 Computational Fluid Dynamies with CFX-10.0 . . . . . . . . 21

3.3 .1 Steady-State Simulation . . . . . . . . . . . . . 21

3.3 .2 Transient Simulations . . . . . . . . . . . . . . . 25

3.4 CFX Output and Post-Processing . . . . . . . . . . . . . . 27

3.4 .1 Force Check . . . . . . . . . . . . . . . . 31

3.4 .2 Moment Check . . . . . . . . . . . . . . 34

3.4 .3 Curve Fitting . . . . . . . . . . . . . . 35

3.5 Curve Fitting in the $y_{0}$ and $z_{0}$ directions . . . . . . . . . . . 41

4 STRUCTURAL FIELD EQUATION MODEL DERIVATION 43

4.1 Kinetic Energy . . . . . . . . . . . . . . . . . . . . . 47

4.2 Potential Energy . . . . . . . . . . . . . . . . . 50

4.3 Equations of Motion . . . . . . . . . . . . . . 52

5 AEROELASTIC TRANSFER FUNCTION 59

5.1 Modal Analysis - Constructing the Eigenfunction . . . . . . . . . . 59

6 CONCLUSION, FUTURE WORK AND RECOMMENDATIONS 73

6.1 Summary ............................... 73

6.2 Conclusion . . . . . . . . . . . . . . . . . 74

6.3 Future Work and Recommendations . . . . . . . . . . . . . 75

$\begin{array}{ll}\text { List of References } & 78\end{array}$

$\begin{array}{lr}\text { Appendix A Matlab post-processing m-file } & \mathbf{8 0}\end{array}$

Appendix B Sample Matlab input data: pressure data for mode 1 at $\begin{array}{ll}\text { timestep } 6501 & 85\end{array}$

$\begin{array}{ll}\text { Appendix C Matlab m-file output for sample input } & \mathbf{8 8}\end{array}$ 


\section{List of Tables}

2.1 NIMONIC alloy 115 material properties at $750^{\circ} \mathrm{C}[1] \ldots \ldots$

2.2 Nodal displacements . . . . . . . . . . . . . . . . 14

2.3 Scaled nodal displacements . . . . . . . . . . . . . . . 15

2.4 Modal transformation representation details . . . . . . . . . . . 16

3.1 CFD input constants . . . . . . . . . . . . . . . . 22

3.2 Initial curve fit constants for $F_{y}$ (mode 1$) \ldots \ldots . \ldots . \ldots$

3.3 Final curve fit constants for $F_{y}$ (mode 1$) \ldots \ldots 38$

3.4 Curve fit constants for $F_{y}$ (mode 1 - half amplitude) . . . . . . . 39

5.1 Curve fitting residual comparison . . . . . . . . . . . . 64

5.2 Roots of the system equation determinant, $k=0 \ldots \ldots 71$

5.3 Roots of the system equation determinant, $k=1 \ldots \ldots 72$

5.4 Roots of the system equation determinant, $k=100,000 \ldots \ldots 72$

viii 


\section{List of Figures}

2.1 Original power turbine blade (variable cross-section) . . . . . . . 8

2.2 Constant cross-section blade . . . . . . . . . . . . . . . 9

2.3 Stress contour plot at blade operating speed . . . . . . . . . . . . 10

2.4 Mode 1 - first bending $(1339 \mathrm{~Hz}) \ldots \ldots . \ldots 11$

2.5 Mode 2 - first torsion $(2676 \mathrm{~Hz}) \ldots \ldots \ldots$

2.6 Mode 3 - first lead $/ \operatorname{lag}(4724 \mathrm{~Hz}) \ldots \ldots . \ldots 12$

2.7 Leading and trailing edge nodes . . . . . . . . . . . . . . 13

2.8 Transformation diagram $\ldots \ldots \ldots \ldots$

3.1 Fluid domain . . . . . . . . . . . . . . . . . . . 19

3.2 CFX-Pre domain with constraints applied . . . . . . . . . 23

3.3 Pressure distribution in the $y_{0}$ direction along the blade periphery for both mesh descriptions . . . . . . . . . . . . . . . . 24

3.4 Pressure as is distribution in the $z_{0}$ direction along the blade periphery for both mesh descriptions . . . . . . . . . . . . . . . . 25

3.5 Steady-state solution pressure profile . . . . . . . . . . . . 26

3.6 Timestep comparison, $F_{y_{0}}$ for mode $1 \ldots \ldots \ldots 28$

3.7 CFX-Solve plot showing the aerodynamic resultant forces and moment as they vary with the timestep $($ mode 1$) \ldots \ldots . \ldots 29$

3.8 Aerodynamic force in the $y_{0}$ direction for mode $1 \ldots \ldots$

3.9 Aerodynamic force in the $z_{0}$ direction for mode $1 \ldots 30$ 
3.10 Aerodynamic moment about the $x_{0}$-axis for mode $1 \ldots 31$

3.11 Pressure profile for mode 1 at timestep $6501 \ldots$. . . . . . . . 33

3.12 Pressure along blade periphery for mode 1 at timestep $6501 \ldots 33$

3.13 Pressure plot along blade periphery for mode 1 at timestep 6501 . . . 34

3.14 Blade cross-section with principal axes, $y$ and $z \ldots . \ldots 36$

3.15 Mode 1 - force in the $y$ direction over time . . . . . . . . . . . 37

3.16 Mode 1 - force in the $y$ direction over time (optimized curve fit) . . . 39

3.17 Mode 1 (half amplitude) - force in the $y$ direction over time . . . . . 40

4.1 Rotating hub-beam system as seen from the positive $Z$-axis . . . . . . 44

4.2 Isometric view of rotating hub-beam system . . . . . . . . . . . 45

4.3 Blade cross-section as seen from the positive $x_{0}$-axis $\ldots \ldots \ldots$

4.4 Blade cross-section with twist about the shear centre . . . . . . . . . 46

4.5 Position vector $r(x, t) \ldots \ldots \ldots \ldots$

4.6 Position vector,$\vec{r}(x, t) \ldots \ldots \ldots$

6.1 Campbell diagram . . . . . . . . . . . . . . . 76

D.1 Mode 1 - force in the $y_{0}$ direction over time . . . . . . . . . 89

D.2 Mode 1 - force in the $z_{0}$ direction over time . . . . . . . . . 90

D.3 Mode 2 - force in the $y_{0}$ direction over time . . . . . . . . . 90

D.4 Mode 2 - force in the $z_{0}$ direction over time . . . . . . . . . . . 91

D.5 Mode 3 - force in the $y_{0}$ direction over time . . . . . . . . . . . 91

D.6 Mode 3 - force in the $z_{0}$ direction over time . . . . . . . . . . . 92 


\title{
Nomenclature
}

\author{
$\dot{\alpha} \quad$ Constant rotational velocity of hub $[\mathrm{rad} / \mathrm{s}]$ \\ $\Delta y_{0} \quad$ Nodal displacement used to create the step change in CFX \\ (in the $y_{0}$ direction) $[\mathrm{m}]$ \\ $\Delta z_{0} \quad$ Nodal displacement used to create the step change in CFX \\ (in the $y_{0}$ direction) $[\mathrm{m}]$ \\ $\delta$ \\ Variation with respect to the deformations, $v$ and $\psi$, and \\ the rotation angle, $\alpha$ \\ $\zeta \quad$ Dummy variable along the $x$-direction $[\mathrm{m}]$ \\ $\eta \quad$ Modal vector \\ $\theta \quad$ Angle between the original and principal axes, $46^{\circ}$ [deg] \\ $\rho \quad$ Density of the hub and beam material $\left[\mathrm{kg} / \mathrm{m}^{3}\right]$ \\ $\tau \quad$ Torque at hub (resisting motion) $[\mathrm{Nm}]$ \\ $\Upsilon \quad$ Matrix entry in the equation of motion \\ $\Phi \quad$ Angle of twist about the trailing edge node [deg] \\ $\psi \quad$ Twist angle of deflection about the shear centre [rad]
}

xi 
Normalized generalized aerodynamic forces and moments acting

on the natural modes

$\psi_{x}$

$\omega$

$\omega_{1}$

$\omega_{2}$

$\omega_{3}$

$\vec{i}$

$\vec{j}$

$\vec{k}$

$\vec{r}$

$\dot{m}$

A

$A(s) \quad$ Transient aerodynamic matrix expressed in the frequency domain

$A(t) \quad$ Transient aerodynamic matrix expressed in the time domain

$A_{b} \quad$ Area of the blade cross-section $\left[\mathrm{m}^{2}\right]$

$a(s) \quad$ Matrix entry in the transient aerodynamic matrix expressed in the frequency domain

$a(t) \quad$ Matrix entry in the transient aerodynamic matrix expressed in 
the time domain

$B \quad$ Factor controlling the rate of logarithmic decrement in the curve fitting $[1 / \mathrm{s}]$

C Factor defining the phase shift in the curve fitting [rad]

$D \quad$ Scaling factor for the curve fitting $[\mathrm{N}$ or $\mathrm{Nm}]$

$E \quad$ Frequency of the arctan function used in the curve fitting $[\mathrm{rad} / \mathrm{s}]$

$E_{b} \quad$ Modulus of elasticity of the hub/blade material [Pa]

F Factor controlling the vertical shift for the curve fitting [N or $\mathrm{Nm}$ ]

$f(t) \quad$ Function that varies with time $[\mathrm{N}$ or $\mathrm{Nm}]$

$F_{\infty} \quad$ Aerodynamic force applied in the $y$-direction [N]

$F_{c} \quad$ Centrifugal stiffening force $[\mathrm{N}]$

$F_{y} \quad$ Resultant aerodynamic force acting on the blade in the $y$ direction $[\mathrm{N}]$

$F_{z} \quad$ Resultant aerodynamic force acting on the blade in the $z$ direction $[\mathrm{N}]$

$F_{y_{0}} \quad$ Resultant aerodynamic force acting on the blade in the $y_{0}$ direction $[\mathrm{N}]$

$F_{z_{0}} \quad$ Resultant aerodynamic force acting on the blade in the $z_{0}$ direction $[\mathrm{N}]$

$G \quad$ Modulus of rigidity of the hub/blade material [Pa] xiii 
I

$I_{S C} \quad$ Moment of area of the blade cross-section about the shear centre $\left[\mathrm{m}^{4}\right]$

$I_{h} \quad$ Moment of area of the hub $\left[\mathrm{m}^{4}\right]$

$J_{S C} \quad$ Polar moment of inertia of the blade cross-section about the shear

j Summation index integer [no unit]

$K \quad$ Stiffness matrix

$k \quad$ Aerodynamic scaling factor [no unit]

$L \quad$ Length of the blade [m]

Mass matrix

$M 1 F_{y_{0}} \quad$ Function approximating the aerodynamic force in the $y_{0}$ direction caused by the blade displacement defined by mode 1

$M 1 F_{z_{0}} \quad$ Function approximating the aerodynamic force in the $z_{0}$ direction caused by the blade displacement defined by mode 1

$M 2 F_{y_{0}} \quad$ Function approximating the aerodynamic force in the $y_{0}$ direction caused by the blade displacement defined by mode 2

$M 2 F_{z_{0}} \quad$ Function approximating the aerodynamic force in the $z_{0}$ direction caused by the blade displacement defined by mode 2

$M 3 F_{y_{0}} \quad$ Function approximating the aerodynamic force in the $y_{0}$ direction 
caused by the blade displacement defined by mode 3

$M 3 F_{z_{0}} \quad$ Function approximating the aerodynamic force in the $z_{0}$ direction caused by the blade displacement defined by mode 3

$M_{\infty} \quad$ Aerodynamic moment applied about the shear centre $[\mathrm{Nm}]$

$N \quad$ Number of nodes considered in the modal analysis

Number of modes considered in the modal analysis

$Q \quad$ Aerodynamic loading vector

$R \quad$ Hub radius [m]

$S_{z} \quad$ Shear centre offset; distance to the $S C$ upstream of the $C O M[\mathrm{~m}]$

$s \quad$ Frequency domain variable

$T \quad$ Kinetic energy [J]

TF Transfer function

$t \quad$ Time $[\mathrm{s}]$

USUM Magnitude of the deflection [m]

$U_{x} \quad$ Deflection in the $x$ direction $[\mathrm{m}]$

$U_{y_{0}} \quad$ Deflection in the $y_{0}$ direction $[\mathrm{m}]$

$U_{z_{0}} \quad$ Deflection in the $z_{0}$ direction [m]

$u \quad$ Nodal displacement vector $\left[10^{-3} \mathrm{~m}\right]$

$V \quad$ Potential energy [J]

$v \quad$ Bending deflection in the $y$-direction [m] 


\begin{tabular}{|c|c|}
\hline$v_{y_{i n}}$ & $\begin{array}{l}\text { Average velocity at the inlet in the } y_{0} \text { direction, (in the steady- } \\
\text { state case) }[\mathrm{m} / \mathrm{s}]\end{array}$ \\
\hline$v_{y_{o u t}}$ & $\begin{array}{l}\text { Average velocity at the opening in the } y_{0} \text { direction, (in the steady- } \\
\text { state case) }[\mathrm{m} / \mathrm{s}]\end{array}$ \\
\hline$W$ & Work done by non-conservative forces $[\mathrm{J}]$ \\
\hline$x$ & Axis pointing spanwise $[\mathrm{m}]$ \\
\hline$y$ & Axis pointing along the minor principal axis of the blade $[\mathrm{m}]$ \\
\hline$y_{0}$ & Axis pointing horizontally upstream of the blade $[\mathrm{m}]$ \\
\hline$z$ & Axis pointing along the major principal axis of the blade $[\mathrm{m}]$ \\
\hline$z_{0}$ & Axis pointing vertically upwards [m] \\
\hline
\end{tabular}

\section{Subscripts}

$\begin{array}{ll}b r & \text { Blade rotation } \\ b t & \text { Blade twisting } \\ c s f & \text { Centrifugal stiffening force } \\ d e n & \text { Denominator } \\ e p & \text { Elastic potential energy } \\ h r & \text { Hub rotation } \\ t r & \text { Transient component of the aerodynamic force } \\ s s & \text { Steady-state component of the aerodynamic force }\end{array}$




\section{Superscripts}

1 Derivative with respect to length, $x$

Derivative with respect to time, $t$

\section{Acronyms}

2-D Two-dimensional

3-D Three-dimensional

CFD Computational fluid dynamics

COM Centre of mass

FEA Finite element analysis

FFT Fast fourier transform

$S C \quad$ Shear centre

SST Shear stress transport

xvii 


\section{Chapter 1}

\section{INTRODUCTION}

The research described in this thesis consists of producing the aeroelastic transfer function for a turbine blade represented as a rotating cantilever beam. The aeroelastic transfer function relates the structural properties of the system to the aerodynamics, and thus provides an aeroelastic definition of the system. This definition allows for a simple calculation to determine the stability and response of the system. As such, the methodology proposed in this thesis is of great significance in the research and development of airfoils, as its use can cut down the time in which it takes to determine the aeroelastic response of a blade. Minimizing the turn-around time for aeroelastic analysis will save research and development dollars.

In order to produce the transfer function, information must be known about the structure as well as the aerodynamics of the system at a given operating condition. The natural modes of vibration of the rotating structure have been determined using a finite element solver. The aerodynamics have been produced using computational fluid dynamics software. The aeroelastic transfer function is calculated by coupling the aerodynamic output with the structural output from the finite element software.

This thesis discusses the method by which the aeroelastic transfer function is obtained. The importance lies in developing this methodology, as it can be used for any internal flow situation. 
This chapter documents the background information and literature review completed.

\subsection{Overview}

Computationally achieving the aeroelastic response of a turbine blade is currently an arduous task. It involves solving the aerodynamic flow field about an airfoil to produce the aerodynamic forces about the blade. At this point a newly deformed blade shape can be calculated as a result of these aerodynamic forces. The flow field will change as the blade shape changes. Therefore, new aerodynamic forces must be calculated as a result of the deformed blade. In this manner, an iterative analysis can be undertaken in order to solve for the aeroelastic response.

The possibility of eliminating the iteration process would greatly reduce the effort required to calculate the aeroelastic response of a given object. A method has been proposed to determine an aeroelastic transfer function that would fully describe this response. This method uses computational fluid dynamics (CFD) analysis and structural equations to determine a transfer function which represents the unsteady aerodynamic effects associated with the impulsive excitation of the structural modes of the blade, based on a given blade shape and initial flow field.

ANSYS-10.0 is used to determine the first three natural mode shapes of a test blade, at the blades' operating speed. Although there are an infinite amount of natural mode shapes, the first three are the highest energy modes, and therefore the most important to consider. These mode shapes are used as displacement inputs into three CFD analyses; one for each shape considered. Each CFD analysis produces the resultant aerodynamic loads on the airfoil over time due to each of the mode shape displacement perturbations.

The next step in the process is to derive the structural field equations for the blade, 
which provides an introduction to the modal analysis that follows. The cantilever beam assumption has been made for simplicity in this first work.

The last step in the process is to produce the transfer function. The transfer function can produce much useful system information, such as whether the system is stable and its response to a general input. This function is formed by combining the aerodynamic loading information with the ANSYS data.

The project consists of performing the aforementioned process with a test blade; a turbine blade designed by Kenny [1]. The structural equations have been produced to demonstrate the theoretical basis of the methodology.

\subsection{Literature Review}

The following is a short summary of the intellectual material that aided in the research. The documents reviewed are summarized each in turn. The first few examine the aerodynamic and aeroelastic information, while the following six papers discuss the derivation of field equations to describe the structural system. The final two papers reviewed concern modal analysis methods.

The AGARD Manual on Aeroelasticity in Axial-Flow Turbomachines contains a thorough review of the history of research done in the research area up until 1987. Volume 1 [2], "Unsteady Turbomachinery Aerodynamics", discusses aerodynamic theory and development over the years. Specific topics discussed are linearized unsteady aerodynamics theory, classical two-dimensional methods, three-dimensional flows, numerical methods for unsteady transonic flow, stall, flutter, unsteady aerodynamic measurements in flutter research, unsteady aerodynamic measurements in forced vibration research, understanding fan blade flutter through linear cascade aeroelastic testing, and unsteady aerodynamic measurements on rotors.

Of specific importance to the current research is the information presented on 
blade instability. Although the manual deals almost exclusively with the compressor blade, the information on modelling and finite element techniques is relevant to the turbine blade also.

The manual discusses interblade phase angle incorporation, inclusion of compressibility in aerodynamic analysis, rotating stall prediction, and increased aeroelastic benefits due to composite blade materials.

The first volume of the AGARD manual concludes with emphasis on the necessity of test-rig experimentation, noting that the cost of which limits the potential to complete such experimentation to large organizations, such as NASA and engine manufacturers, in most cases.

Volume 2 of the AGARD manual is entitled "Structural Dynamics and Aeroelasticity" [3]. This document reviews the structural modelling methods used for both single blades, and blade-disk assemblies, as well as the analytical and experimental methods used to describe the aeroelastic system. The discussed topics are: basic structural dynamics (single and multi-blade configurations), fatigue and assessment methods of blade vibration, lifetime predictions, analytic aeroelastic coupling and formulations, fan flutter experimentation, thermal effects, forced vibration and flutter design methodology.

The widespread use of finite element methods in the structural considerations is noted and the useful element types are described (for both beam-type and plate shell elements). Although the finite element method is quite useful, the benefit of deriving the governing set of differential equations is mentioned with reference to the use of aerodynamic data as the loading data.

The second volume of the AGARD manual closes much as the first volume, in stating that experimentation in aeroelasticity is paramount to achieving a stable design.

The next document reviewed was written by Schroeder and Fleeter [4]. In this 
document the authors develop a mathematical model for predicting unsteady aerodynamics of a flat plate airfoil. The plate exists in laminar flow at moderate Reynolds numbers and is executing harmonic torsional motion. This paper assumes the vibration of the blade to occur without change as the aerodynamic loads vary.

The following six papers involve the derivation of the structural field equations for a rotating cantilever beam. The paper by Yang and Chen was used as a guideline to obtain the equations of motion for the rotating turbine blade in Chapter 4 .

Yang and Chen [5] write about the control of a beam's transverse vibrations. This article examines a flexible Euler-Bernoulli beam fixed to a rigid rotating hub. The Euler-Bernoulli beam allows for quick calculation by approximating the beam by neglecting the effects of rotational lateral inertia. Deformation (axial, transverse, and rotational) is restricted to the plane of rotation, and the only non-conservative force is the torque creating the angular motion. The structural equations and model constraints are derived using Hamilton's Principle, and include the effects of centrifugal stiffening. The equations are then transformed into finite element notation. The remainder of Yang and Chen's study involves active vibration control.

$\mathrm{Hu}$ and Vlahopoulos [6] introduce a new methodology for modelling a flexible rotating beam by finite elements. Their study includes both rigid and flexible motions. The energy method (Lagrange) is used to model the system. Hu and Vlahopoulos study is completely structural and includes the potential energy due to centrifugal stiffening.

Yau and Fung [7] study a rotating flexible arm which is clamped-free and rotating in the horizontal plane. The clamped-free condition has a cantilever connection at the hub and an restrained blade tip. The arm is carrying a mass which is not stationary. Their beam is modelled as an Euler-Bernoulli beam, but they ignore shear deformation effects.

Fung and Yau [8] present a study on the effect on the vibration frequencies by the 
centrifugal stiffening effect. The test subject is a clamped-free rotating flexible EulerBernoulli beam. The equations of motion and the model constraints are determined using Hamilton's Principle.

Cai et al. [9] have also studied active control. Their test model, a rotating flexible cantilever, is derived using Hamilton's Principle.

Zibdeh and Juma [10] examine a beam using Euler-Bernoulli, Rayleigh and Timoshenko beam models. Their problem is completely structural and involves the transverse vibrations of a homogenous isotropic rotating beam.

Coupled analysis has been studied by Jacquet-Richardet and Henry [11], who have produced a method for flutter analysis of rotating blade assemblies using a modal method. The structure is modelled using finite element theory. When applicable, the aerodynamics were assumed constant along the blade span using the midspan measured value. An interesting outcome of this research was the determination that chordwise bending is significant in low aspect ratio blades, and should not be neglected. This method does not solve for the aerodynamic forcing functions, but approximates them. An aeroleastic transfer function is not calculated in this paper.

The last paper reviewed regarded reduced-order models. These models have been introduced in recent years to examine aircraft flutter. Silva [12] discusses the use of reduced-order models in external systems. Modern papers on aeroelastics in external flow are based on Silva's work and have therefore not been included in this literature review. Note that these works deal with external flows. The novelty in the current thesis lies in applying the methods used for external flows on internal flows.

According to the above-mentioned review, and to the knowledge of the author, there is no paper that produces a simple aeroelastic, although the same concept has been explored for external flows. The following work is completely new, and novel. 


\section{Chapter 2}

\section{NATURAL MODE SHAPE CALCULATION USING ANSYS-10.0}

The purpose of this chapter is to produce the first three natural mode shapes for a turbine blade rotating at its operating speed. The first step is to create a solid model of the test blade for use in the finite element analysis (FEA) software, ANSYS-10.0. The ANSYS output is post-processed for input in the subsequent CFD analyses. The governing equations used in the ANSYS simulations can be found in the associated software manual [13].

The turbine blade in question was taken from the power turbine designed by Kenny [1]. This blade was modified to have a constant cross-section from root to tip. The material properties have been taken from Kenny [1] and are shown in Table 2.1.

Table 2.1: NIMONIC alloy 115 material properties at $750^{\circ} \mathrm{C}[1]$

\begin{tabular}{|c|c|}
\hline \multicolumn{2}{|c|}{ NIMONIC alloy 115} \\
\hline \hline Density $\left[\mathrm{kg} / \mathrm{m}^{3}\right]$ & 8190 \\
\hline Yield Strength $[\mathrm{MPa}]$ & 750 \\
\hline Ultimate Tensile Strength $[\mathrm{MPa}]$ & 1000 \\
\hline Young's Modulus [GPa] & 179.5 \\
\hline Poisson's Ratio & 0.317 \\
\hline
\end{tabular}


The first step in this analysis was to modify the solid model obtained from Kenny [1] to produce a constant cross-section model. With this modification the centre of mass of any cross-section of the blade is located at the same $y_{0}$ and $z_{0}$ positions. This simplification is helpful when defining the nodal deflection matrix, $[u]^{T}$, for the aeroelastic analysis, shown in Chapter 5. This process was performed using Pro/Engineer.

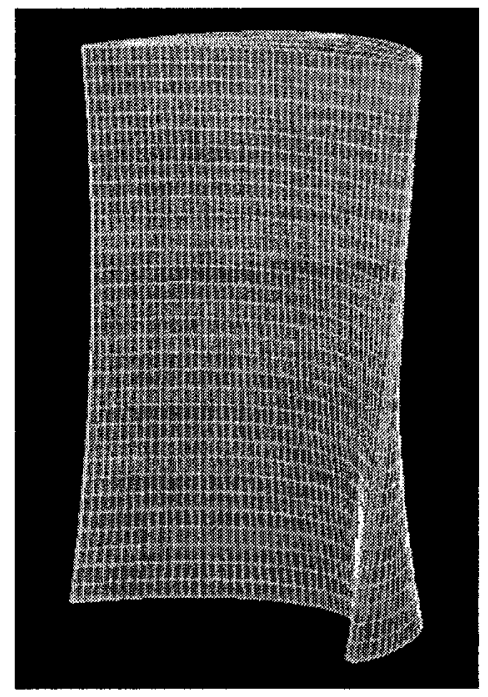

Figure 2.1: Original power turbine blade (variable cross-section)

The blade model created in Pro/Engineer was inputted into ANSYS. It was within ANSYS that the blade is meshed into finite elements, given material properties, and structurally loaded.

Several solid element types are available in the ANSYS software. An element type must be chosen and given material properties prior to the meshing process. Brick shaped elements, often using 20 nodes per element, are a suitable choice for a stress or modal solution [14]. This element class includes four basic element types; 20-node hexahedral, 10-node tetrahedral, 15-node prism, and 13-node pyramid elements.

As the material properties of the blade were known, they were programmed into 
ANSYS before any analysis took place. A new material model was defined by inputting all the properties that are known. In this case, the Young's Modulus, Poisson's Ratio, the yield strength, and the density were the values required for the analysis. This material description was called 'Material 1'.

The constant cross-section blade had a relatively simple shape and could be easily meshed using the ANSYS automatic mesher. The automatic mesher requires few inputs; the element type, the material model, the global mesh size, and the part to be meshed. Using the previously discussed brick elements and 'Material 1' with a global mesh size of 3 , the entire blade model was meshed. The resulting elemental structure is shown in Figure 2.2. Note that the results were calculated using two mesh densities, one with 9,731 elements, and the other with 32,241 elements. The results for the two mesh cases were the same. The finer mesh $(32,241$ elements) was used as did not significantly increase the computation time.

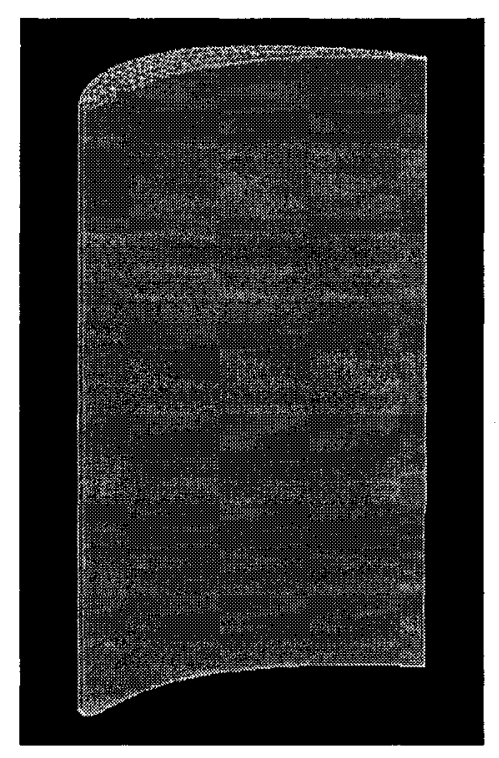

Figure 2.2: Constant cross-section blade

With the element structure defined, the model was spatially restrained. In accordance with the initial assumptions, the blade was treated as a cantilever beam. 
The root was therefore to be restrained in all directions. ANSYS accomplished this situation by defining the root plane to have no deflection in any direction. The root had the only spatial restriction. Although the hub will deform in a real situation, the importance of this work lies in presenting the methodology, and therefore, this time-saving simplification was made.

The turbine is assumed to spin at a constant speed. Therefore, the ANSYS model was assigned the same inertial loading. This was accomplished by defining a global rotational velocity about the $Z$-axis (2880 rad/s or $27,500 \mathrm{rpm}$ ). Figure 4.5 illustrates the orientation of the $Z$-axis.

Determining the vibration modes at the operating point required two analyses. First the steady-state stress analysis was solved so it could be included in the modal analysis. At this stage the blade model was completely defined, and the first simulation could be run. The stress state can be seen in Figure 2.3.

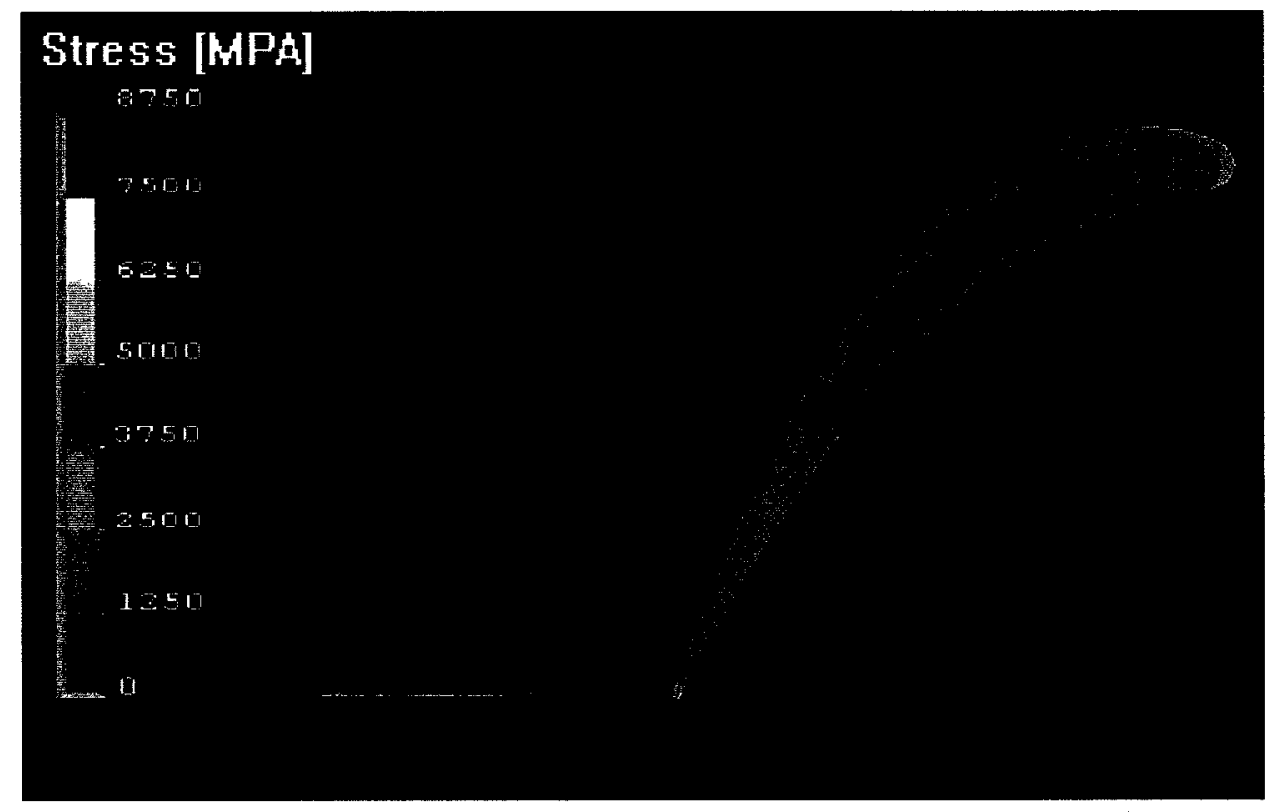

Figure 2.3: Stress contour plot at blade operating speed

The trailing edge is the thinnest region, so it was expected that the maximum stress would occur at that location. As expected, the maximum stress was seen at 
the trailing edge of the blade at the root. The value of the maximum stress was 3,795 MPa.

With the stress state due to rotation known, the modal analysis at the operating speed could be performed. It was the first three natural mode shapes that were being sought. Figures 2.4, 2.5 and 2.6 illustrate the results of the modal analysis.

The first mode existed at a frequency of $1339 \mathrm{~Hz}$. This mode was primarily bending. The second mode was the first torsion mode, and it was found to exist at $2676 \mathrm{~Hz}$. The first lead-lag mode occurred at $4724 \mathrm{~Hz}$ and was the third natural frequency of the blade rotating at $27,000 \mathrm{rpm}$.

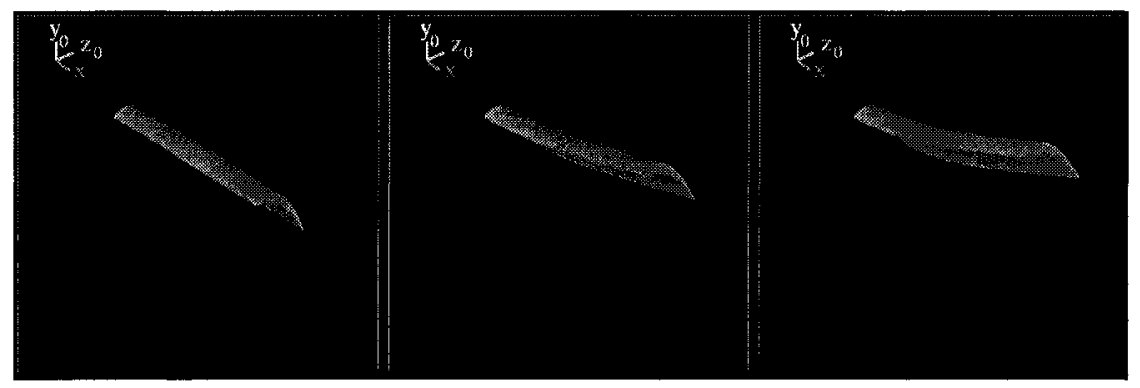

Figure 2.4: Mode 1 - first bending $(1339 \mathrm{~Hz})$

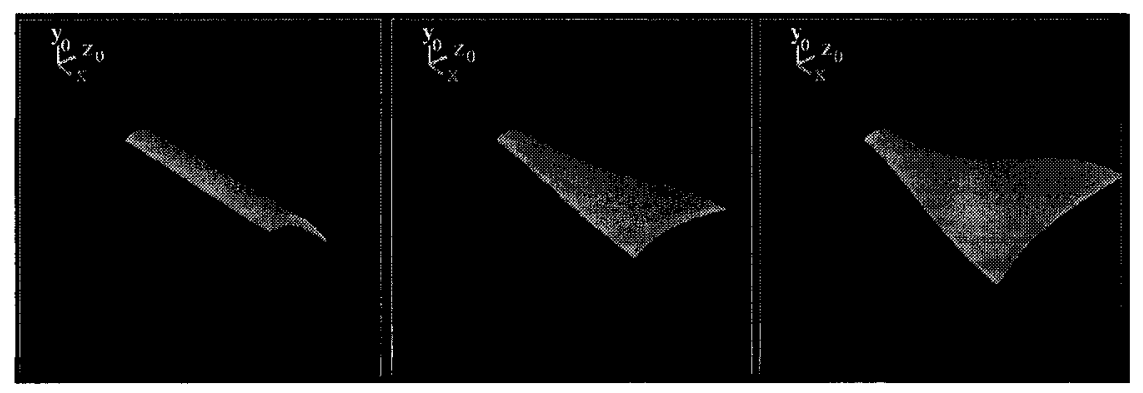

Figure 2.5: Mode 2 - first torsion $(2676 \mathrm{~Hz})$

The goal of the ANSYS analysis was to provide natural mode shapes to be inputted into the CFX simulations. The CFX simulations have been treated as 2-D computational fluid dynamics analyses conducted at a cross-section located at the 


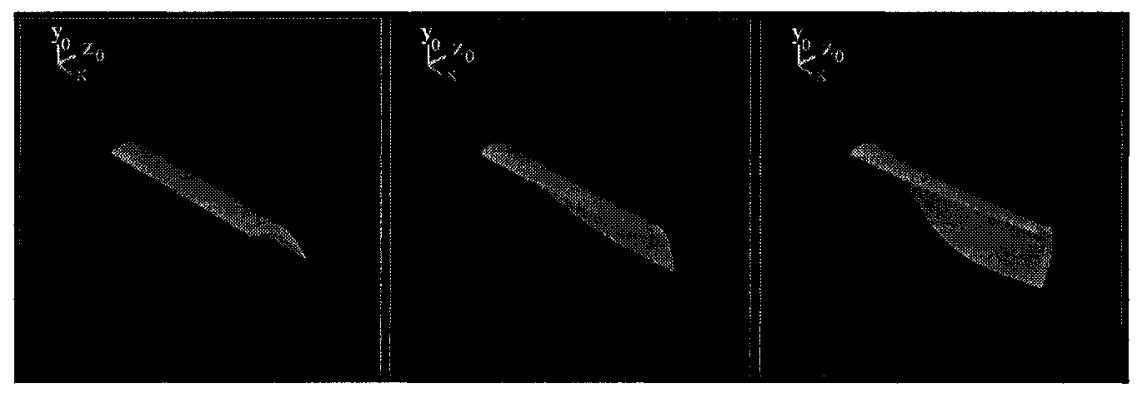

Figure 2.6: Mode 3 - first lead/lag (4724 Hz)

blade midspan. Therefore, it was necessary to represent the mode shapes in a form compatible with the CFX program.

The CFD analysis considered only the midspan cross-section. To simplify the analysis the cross-section was assumed to remain plane. It was also assumed that the shape of the cross-section remains constant. This assumption was necessary as there is no way to model out of plane motion in a 2-D analysis. With these assumptions the midspan blade displacement, for each mode shape, was the only requirement needed from the ANSYS simulations.

The non-deformable cross-section assumption allowed for a simple method for determining the displacement at the blade midspan. It was a matter of choosing two nodes on the cross-sectional area and attaining their respective displacements. The nodes chosen represent the leading and trailing edges; these are the nodes whose $z_{0^{-}}$ coordinates are the cross-section maximum and minimum values, Figure 4.5 illustrates the orientation of the $z_{0}$-axis. These nodes are shown in Figure 2.7.

The first three modes exhibited the displacements shown in Table 2.2 at the leading and trailing edge nodes. The $U x, U y_{0}$ and $U z_{0}$ columns in this table represent the deflections in the $x, y_{0}$ and $z_{0}$ directions, respectively. The deflections acquired from the modal simulation became inputs into the aerodynamic simulations that followed.

The amplitudes of the each of the structural mode shapes has been determined by normalizing the nodal deflection matrix to the mass matrix. The value of the 


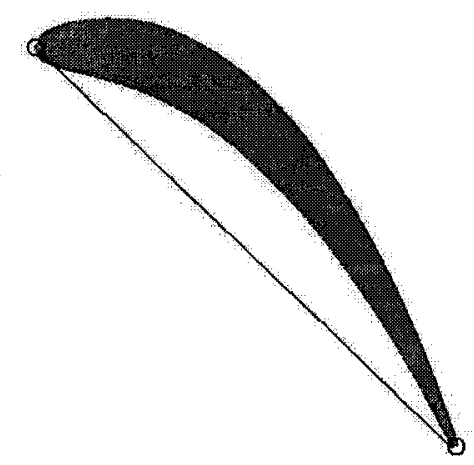

Figure 2.7: Leading and trailing edge nodes

total deflection due to each mode is not important, but the direction of the motion is important, i.e., the relationship between the deflections in the $x, y_{0}$ and $z_{0}$ directions. As such it was possible to scale the amplitude of the mode shapes in order best suit the fluid response simulations.

The fluid response simulations required that the step input provided by the structural mode shapes was large enough to elicit a transient fluid effects. At the same time, the amplitude of the step input must be small enough to maintain a linear relationship in the fluids (i.e., doubling the step input doubles the transient fluid response). The value chosen was one-hundredth of the blade chord length. This value produced transient effects in the fluid, while keeping the transient portion of the aerodynamic response close to the linear range.

The modes were scaled by one-hundredthh of the blade chord, and are therefore presented in meters. The USUM column is the magnitude of the sum of the vector addition of the individual deflections. The last column in Table 2.2 shows the percentage of the total deformation that occurs in the $x$ direction, the spanwise direction.

Remembering that the cross-sectional plane is the $z_{0}-y_{0}$ plane, it should be noted that the maximum displacement in the spanwise direction, the $x$ direction, was $28.7 \%$ of the total value. The $x$ direction nodal displacement has been neglected, as stated above. Unfortunately this was a large percentage of the blade displacement, and 
will most likely cause errors in the resulting CFD analysis. As this work is aimed at proving a methodology, this issue is not of great concern. The possibility of extending this 2-D analysis method into 3-D analysis is quite possible, and this extension would correct what has been neglected in this first work.

Table 2.2: Nodal displacements

\begin{tabular}{|l|c|c|c|c|c|}
\hline & $\begin{array}{c}U x \\
\left(10^{-3} \mathrm{~m}\right)\end{array}$ & $\begin{array}{c}U y_{0} \\
\left(10^{-3} \mathrm{~m}\right)\end{array}$ & $\begin{array}{c}U z_{0} \\
\left(10^{-3} \mathrm{~m}\right)\end{array}$ & $\begin{array}{c}U S U M \\
\left(10^{-3} \mathrm{~m}\right)\end{array}$ & $\begin{array}{c}\% U \text { SUM } \\
\text { by } U x\end{array}$ \\
\hline Mode 1 - 1st Bending \\
\hline Leading Edge Node & -22.85 & -72.52 & 77.03 & 108.2 & $4.46 \%$ \\
\hline Trailing Edge Node & -20.07 & -89.96 & 90.46 & 129.1 & $2.42 \%$ \\
\hline Mode 2 - 1st Torsion \\
\hline Leading Edge Node & 6.595 & 228.1 & -50.45 & 233.7 & $0.08 \%$ \\
\hline Trailing Edge Node & 8.227 & -191.7 & 360.2 & 408.1 & $0.04 \%$ \\
\hline Mode 3 - 1st Lead/Lag & & & & \\
\hline Leading Edge Node & -63.06 & 14.21 & 98.46 & 117.8 & $28.7 \%$ \\
\hline Trailing Edge Node & 94.27 & 190.8 & -87.51 & 230.1 & $16.8 \%$ \\
\hline
\end{tabular}

Each mode shape was used as a perturbation in a CFD simulation. As stated previously, the magnitude of the normalized mode shapes needed to be large enough to cause transient effects in the fluid solutions, but small enough to maintain a linear relationship between the input and the resulting fluid field. A linearity check was performed using a maximum deflection of one-hundredth of the blade chord and is shown in Chapter 3. As such, the maximum displacement was set to one-hundredth of the blade chord, approximately $2.5 \times 10^{-4} \mathrm{~m}$. The next step was to set the maximum nodal displacement for each mode to $2.5 \times 10^{-4} \mathrm{~m}$. This amplitude change produced the following scaled nodal displacements.

The mode shapes could be expressed simply as a translation and subsequent rotation from the undisplaced to the displaced state. Figure 2.8 illustrates the way in 
Table 2.3: Scaled nodal displacements

\begin{tabular}{|l|c|c|c|c|}
\hline & $\begin{array}{c}U x \\
\left(10^{-3} \mathrm{~m}\right)\end{array}$ & $\begin{array}{c}U y_{0} \\
\left(10^{-3} \mathrm{~m}\right)\end{array}$ & $\begin{array}{c}U z_{0} \\
\left(10^{-3} \mathrm{~m}\right)\end{array}$ & $\begin{array}{c}U S \mathrm{SM} \\
\left(10^{-3} \mathrm{~m}\right)\end{array}$ \\
\hline Mode 1 - 1st Bending \\
\hline Leading Edge Node & 0 & -0.1421 & 0.1510 & 0.2073 \\
\hline Trailing Edge Node & 0 & -0.1763 & 0.1773 & 0.2500 \\
\hline Mode 2 - 1st Torsion \\
\hline Leading Edge Node & 0 & 0.1397 & -0.0309 & 0.1431 \\
\hline Trailing Edge Node & 0 & -0.1174 & 0.2207 & 0.2500 \\
\hline Mode 3 - 1st Lead/Lag & & \\
\hline Leading Edge Node & 0 & 0.0169 & 0.1172 & 0.1184 \\
\hline Trailing Edge Node & 0 & 0.2272 & -0.1042 & 0.2500 \\
\hline
\end{tabular}

which a mode shape is described. To begin, both nodes, for a single mode shape, were translated by the trailing edge nodal displacement value. The translation produced the 'TRANSLATED' line in Figure 2.8.

A rotation about the trailing edge node completed the transformation from undisplaced to displaced nodal coordinates. The rotation occurred about the trailing edge node and its value was easily calculated with a right angle triangle, as shown in Figure 2.8 .

In the above-mentioned fashion, each mode shape was expressed as a translation and subsequent rotation about the displaced trailing edge node. Table 2.4 presents the values that define these mode shapes.

Table 2.4 completes the natural structural mode shape definition. These mode shapes were inputted into the CFX-10.0 software to produce the resulting unsteady aerodynamics. This is discussed in the next chapter. 


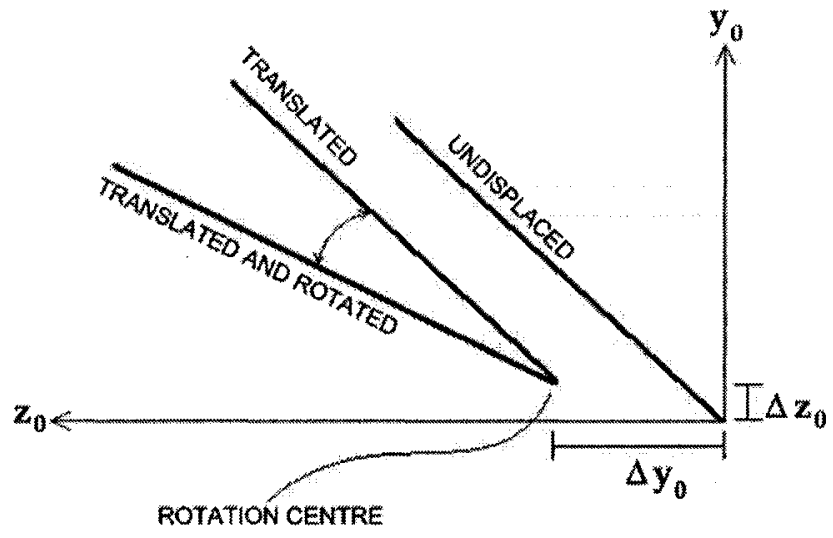

Figure 2.8: Transformation diagram

Table 2.4: Modal transformation representation details

\begin{tabular}{|c|c|c|c|c|}
\hline & $\begin{array}{c}y_{0} \text {-Translation } \\
\left(10^{-3} \mathrm{~m}\right)\end{array}$ & $\begin{array}{c}z_{0} \text {-Translation } \\
\left(10^{-3} \mathrm{~m}\right)\end{array}$ & $\begin{array}{c}\text { Coord. of Rotation } \\
\text { Centre }\left(10^{-3} \mathrm{~m}, 10^{-3} \mathrm{~m}\right)\end{array}$ & $\begin{array}{c}\text { Rot. Angle } \\
(\mathrm{deg})\end{array}$ \\
\hline Mode 1 & -0.1763 & 0.1773 & $(-15.5763,-10.8327)$ & 0.0828 \\
\hline Mode 2 & -0.1174 & 0.2207 & $(-15.5174,-10.7893)$ & 0.6934 \\
\hline Mode 3 & 0.2272 & -0.1042 & $(-15.1728,-11.1142)$ & -0.5872 \\
\hline
\end{tabular}




\section{Chapter 3}

\section{TRANSIENT AERODYNAMIC FORCE DETERMINATION USING CFX-10.0}

The purpose of this chapter is to produce the time dependent aerodynamic force equations that act on a blade as a result of a prescribed blade movement within the blade passage. The prescribed movement of the blade was described by the natural mode shapes of the test blade which were determined in the previous chapter.

In Chapter 2, three main mode shapes were determined. These three mode shapes each required a computational fluid dynamic (CFD) simulation to solve the flow field over time. The ultimate goal was to produce the generalized aerodynamic force expressions in the time domain for each of the first three modes of vibration.

The first step in the process was to define the blade and blade passage dimensions to produce a fluid domain that could be meshed into finite elements. The operating parameters of the system were applied and the steady-state fluid solution was determined.

Transient simulations track the flow field through time, and the solved steadystate flow field was used as a starting point for the transient case. The transient simulations ran for several timesteps until a forced step deformation of the blade occurred, corresponding to the natural mode shapes determined from the ANSYS 
work. A step input excites all of the aerodynamic modes at once. For this reason, a step function was used to excite the aerodynamic modes in the following CFD simulations.

The last step in the work was post-processing the data. The three transient simulations produced much information about the system over time. This information was manipulated to produce equations for aerodynamic force in the $y_{0}$ and $z_{0}$ directions resulting from the flow around the blade.

This chapter describes the process of constructing the CFD simulations, and postprocessing the output to produce information that will be used in conjunction with the ANSYS structural results to produce the aeroelastic transfer function.

The first step in producing the aerodynamic data was determining a fluid domain that would accurately describe the problem. The fluid domain creation is discussed in the following section.

\subsection{Fluid Domain}

The fluid domain was created in order to define a small sliver of volume in one blade passage of the test turbine disk (at half of the blade span, i.e., midspan). The blade profile was the predetermined shape defined by Kenny [1].

The complete domain was constructed using the blade shape at the midspan, the midchord line, the distance between adjacent blades and the axial blade length. The domain used is shown in Figure 3.1, with the interblade interfaces one pitch apart. The domain upstream of the blade is 1.2 times the axial chord length, while the outlet of the domain is located one axial-chord length downstream of the trailing edge.

Although this work only requires a 2-D flow solution, as there should be no flow in the spanwise direction, the CFX program requires a 3-D domain. Therefore, the fluid region was giving a small thickness of $0.00025 \mathrm{~m}(0.25 \mathrm{~mm})$, which is less than 


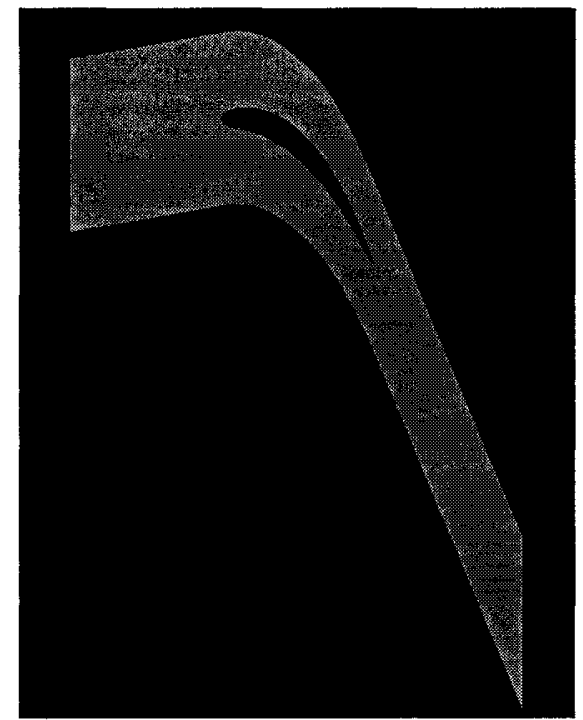

Figure 3.1: Fluid domain

$2 \%$ of the domain length.

At this stage, the domain has been created and is ready can be meshed. There are two options for meshing; structured and unstructured. The next section discusses how the mesh of the domain was created.

\subsection{Mesh Creation}

In order for the CFD software to solve for the flow field in a given domain, the domain must be broken down into finite elements. There are two main types of meshes that can be created; structured and unstructured. Structured grids consist of hexahedral elements (brick form). Unstructured grids utilize bricks as well other types of elements such as tetrahedrals and triangular prisms. The following sections describe the structured and unstructured meshing attempts. 


\subsubsection{Structured Grid}

A structured grid is generally known to provide a more accurate solution to CFD problems [15]. Unfortunately, without a simple geometry, producing a structured grid can be very difficult.

Attempts were made to mesh the domain shown in Figure 3.1 using an O-grid structured mesh, in order to account for the solid body resting in the centre of the flow field. This solid body is the blade, and has a slender trailing edge. This slender portion produced large problems in creating a structured grid.

The unsuccessful nature of the structured grid creation makes it necessary to resort to an unstructured grid. Although the results will be less accurate with an unstructured grid, the focus on this work is to introduce a method, and not to produce exact results. Therefore, the extra time that would be required for the determination of a structured grid was unwarranted.

\subsubsection{Unstructured Grid}

The unstructured grid option, although not the first choice, has been employed in this work. The meshing took place in CAD2Mesh program, which is a meshing program within the ANSYS 9.0 Workbench software. The mesh produced has a boundary layer around the solid blade section with smaller elements at the blade surface. The region just downstream of the trailing edge has been given a cluster of smaller elements to capture the wake.

The mesh created consisted of 20,742 elements. This number includes over fifteen thousand wedge elements and over five thousand prismatic elements. This volume mesh provides a discretized domain for the computational fluid solver.

The meshed domain and the system parameters are the inputs that are required to create the CFX simulation definition. The steady-state simulation definition is 
described in the following section.

\subsection{Computational Fluid Dynamics with CFX- 10.0}

Computational Fluid Dynamics (CFD) is a tool used to model complicated systems. The process involves taking a complex problem and dividing it up into a finite number of simpler problems that a computer can solve. CFX-10.0 is one such CFD program. The governing equations used in the following simulations can be found in the associated software manual [16].

The following sections describe the process of defining and running the steadystate and transient simulations for the rotating turbine blade.

\subsubsection{Steady-State Simulation}

Although it is desired to use a transient simulation to determine the aerodynamic effects over time for each mode, it is necessary to use a steady-state simulation to begin. This is due to two main factors; acquiring an initial state solution and ensuring grid convergence.

A transient solution is best obtained with a solved steady-state solution which can be used as the initial flow field for the transient simulation. Producing this steadystate solution is a simple matter of importing the meshed domain into CFX-Pre and defining its boundaries and initial conditions. Table 3.1 defines the values used in the steady-state problem definition. As the domain is a slice of a blade passage for a rotating turbine disk, the velocity used as the inlet velocity is a function of the rotating speed of the disk and the spanwise location of the slice. At the blade midspan the velocity upstream of the blade is $168 \mathrm{~m} / \mathrm{s}$ with a direction of 14 degrees 
to the blade metal angle (based on Kenny's work, [1]).

Table 3.1: CFD input constants

\begin{tabular}{|c|c|}
\hline Variable Name & Value \\
\hline Inlet Velocity & $168 \mathrm{~m} / \mathrm{s}$ \\
Inlet Flow Angle & 14 degrees \\
Static Pressure at Opening & $90 \mathrm{kPa}$ \\
Reference Pressure & $0 \mathrm{kPa}$ \\
Timestep & $6 \times 10^{-7}$ seconds \\
Fluid & Air as Ideal Gas \\
Inlet Temperature & $945^{\circ} \mathrm{C}$ \\
Outlet Temperature & $755^{\circ} \mathrm{C}$ \\
\hline
\end{tabular}

The timestep value shown in Table 3.1 was calculated using the Courant number. The Courant number relates the average element size in the mesh to the timestep and velocity in the simulation to produces a scalar, unitless number. It is important to keep this number between 0 and 1 . This restriction ensures that as a specific particle in the flow advances through the domain, it will not completely traverse an element in the mesh over a single timestep.

To complete the simulation specification, the domain walls were defined as an inlet, opening, wall, symmetric or periodic boundaries. The defined domain is shown in Figure 3.2. The blade exterior edges are all no-slip walls as they are solid boundaries. The inlet and opening are easily seen as the left-most and right-most boundaries, respectively. The edges that cut through the span of the blade are defined as symmetric boundaries. This definition is chosen as there should be no flow through these boundaries. The top and bottom edges are placed halfway between adjacent blades on a turbine rotor. Therefore, any flow that exits one must enter the other. By this rationale, the top and bottom surfaces are defined to be periodic boundaries. With all of the variables and boundaries defined, the steady-state simulation could be run 


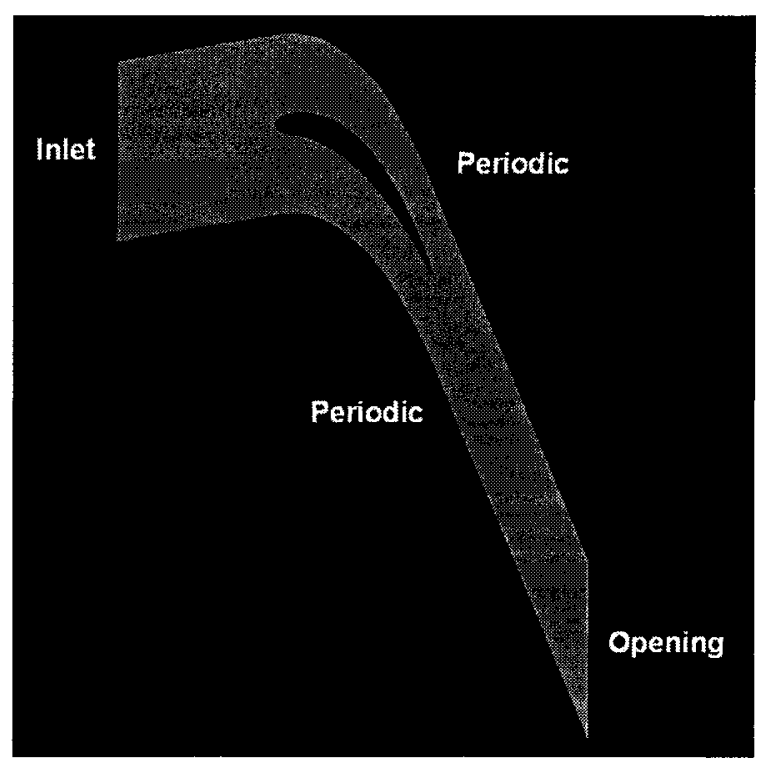

Figure 3.2: CFX-Pre domain with constraints applied

to achieve a solution for the fluid domain about the turbine blade at the operating speed.

The solution of the steady-state simulation produced output that defines the initial state for the transient simulation that followed. It is important for this initial state to be accurate, as this ensures that the transient simulations begin with an accurate description of the flow field. An important checkpoint to verify the validity of the output is to perform a grid convergence test. A grid convergence or mesh refinement study is the process of determining the mesh density required to produce a solution that does not vary with mesh count and mesh distribution. If there are too few elements (large element size), the solution will not be accurate. With a very high number of elements (small element size), the computational time required to produce a solution is excessive. It is desired to have an element count that is small enough to produce an accurate solution, but large enough to minimize the time required to achieve that solution. This check was performed using the original mesh produced and a variation on that mesh. 
The mesh that was determined in Section 3.2.2 is the starting point in the grid convergence analysis. First, the initial mesh (approx. 20,000 elements) was used in a steady-state simulation and the results were outputted. Next, the mesh was modified to contain roughly twice as many elements (approx. 40,000 elements). The mesh with the most elements is referred to as the Fine Mesh; while the original mesh is called Coarse Mesh. The same simulation was rerun with the Fine Mesh. The output for the simulations were then compared. The pressure distribution as it varies along the blade periphery in the $y_{0}$ and $z_{0}$ directions are shown in Figures 3.3 and 3.4 .

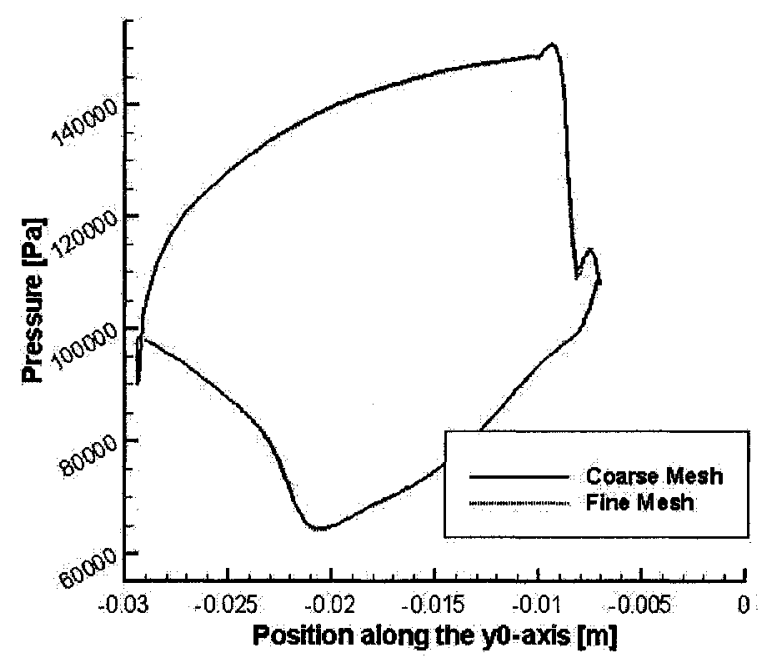

Figure 3.3: Pressure distribution in the $y_{0}$ direction along the blade periphery for both mesh descriptions

The similarity of the output for the Coarse Mesh to that of the Fine Mesh proves that between the two element sizes, the solution is independent of the element size. As such, the Coarse Mesh was chosen for further use to minimize the computational time required.

The meshed domain was used in conjunction with the system values to produce a steady-state simulation definition in the CFX-Pre program. This definition file was run in CFX-Solve until the program achieved a solution for the flow field. The pressure profile of the solution is shown in Figure 3.5. The solved steady-state solution 


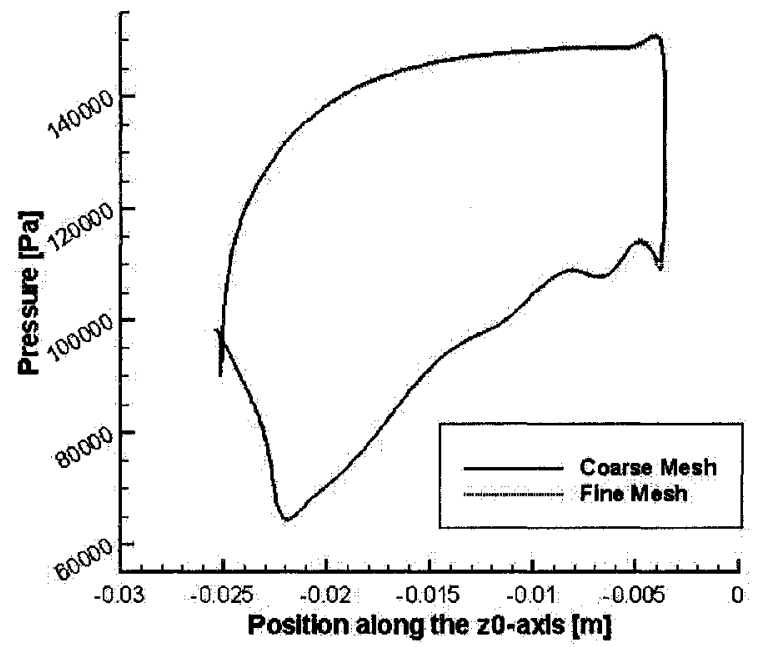

Figure 3.4: Pressure as is distribution in the $z_{0}$ direction along the blade periphery for both mesh descriptions

for the flow field provided an initial state for the transient simulations. The transient simulations are discussed in the section which follows.

\subsubsection{Transient Simulations}

The research depends highly on achieving the resulting aerodynamic force data which occurs as a result of the natural mode shape deflections of the blade. The natural mode shapes were determined in Chapter 2, and post-processed in such a way as to make it easy for them to be inputted into the CFX-Pre program. This section discusses the transient simulations that were conducted using CFX-10.0 to capture the unsteady flow field resulting from the impulsive excitation of the first three natural mode shapes of the test blade at its operating speed. Note that the following describes the first natural mode shape only; the second and third were set up and run in a similar manner.

The steady-state definition file was modified to produce a transient simulation definition. As such, the boundary definitions and initial conditions were already defined. The simulation type was changed to transient with an Shear Stress Transport 

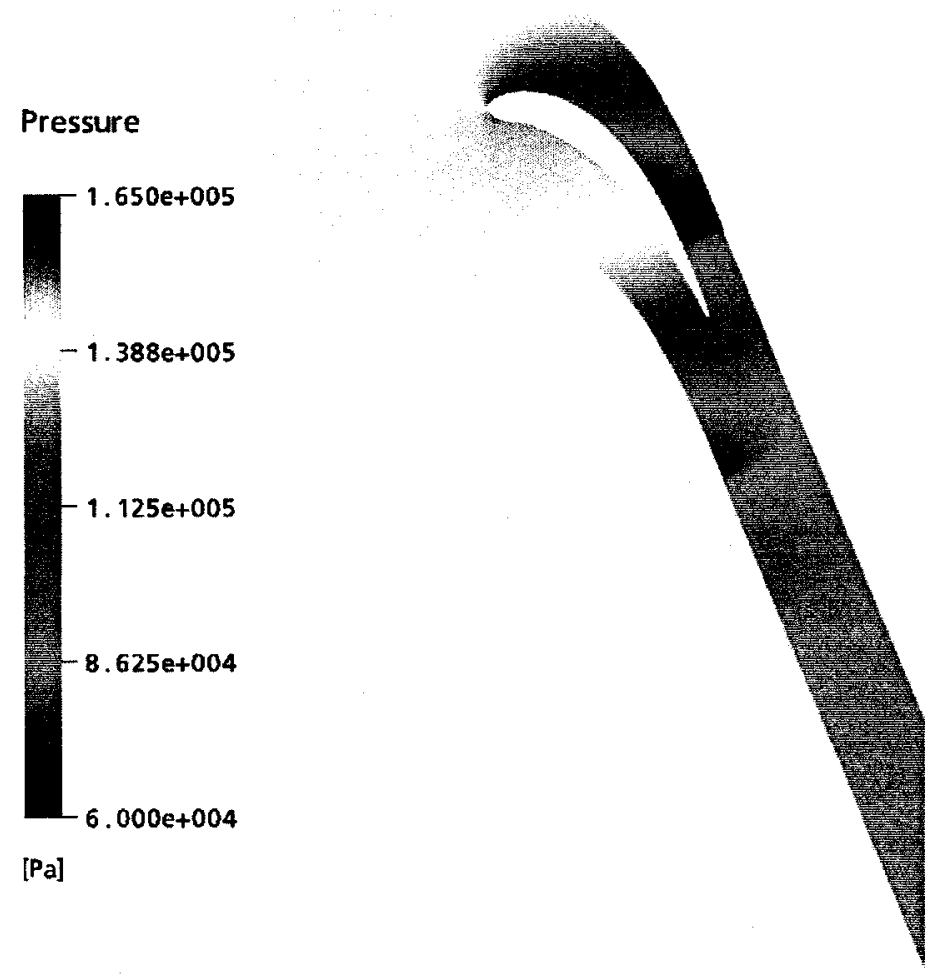

Figure 3.5: Steady-state solution pressure profile

(SST) turbulence model (SST is a common choice for flow with separated boundary layers). Note that the second order backward Euler transient scheme was used [16].

The most important change was made to the blade walls. A built-in capability in CFX-10.0 was used to move the blade walls. To mimic the natural mode shapes, the walls were assigned a rotation angle about the trailing edge node and a general plane displacement in the $y_{0}$ and $z_{0}$ directions. These transformations change the angle of attack of the blade as well as its placements in the blade passage, while keeping the blade shape and size unchanged. These transformations were implemented over a single timestep indicating a step change in the blades orientation and location.

The step change defined for each natural mode was applied just after the tenth timestep in the transient simulation. The simulation continued to run until the results 
stabilized; over five thousand additional timesteps. Note that the timestep used for the transient simulations was the same as that chosen for the steady-state simulations.

The timestep in a CFD problem is a key factor in the accuracy of the output. If the timestep is too small, the simulation will take an excessively long time to reach a solution. Conversely, if the timestep is too large the solution will be reached much quicker, but the data may not be representative of the true solution. A compromise must be made to achieve a timestep that provides accurate data with minimized computational run time. The timestep used in this problem must be checked for its validity.

The timestep chosen in the steady-state simulations was $6 \times 10^{-7}$ seconds. This value was halved and inputted as the timestep value for a transient simulation with all other values remaining unchanged. A snapshot of the transient simulation data is shown in Figure 3.6 for both timesteps. The results for this new simulation were consistent with that achieved with the larger timestep. The major difference in the two curves is seen in this snapshot just after the blade perturbation. The remainder of the results for both timestep values were identical.

This indicates that the transient flow field solution was independent of the timestep for any value between these two timestep values. To reduce the computation time the larger timestep value was chosen. The results of the transient simulation, for mode 1, are shown in Figure 3.7. The last step in the analysis was to post-process the output to produce the information required for coupling with the ANSYS output.

\subsection{CFX Output and Post-Processing}

The CFD program produces a flow field in the blade passage over time. It is up to the operator to interpret this output in a meaningful way. This section describes what the CFX program provided, and how this data was manipulated to produce the 


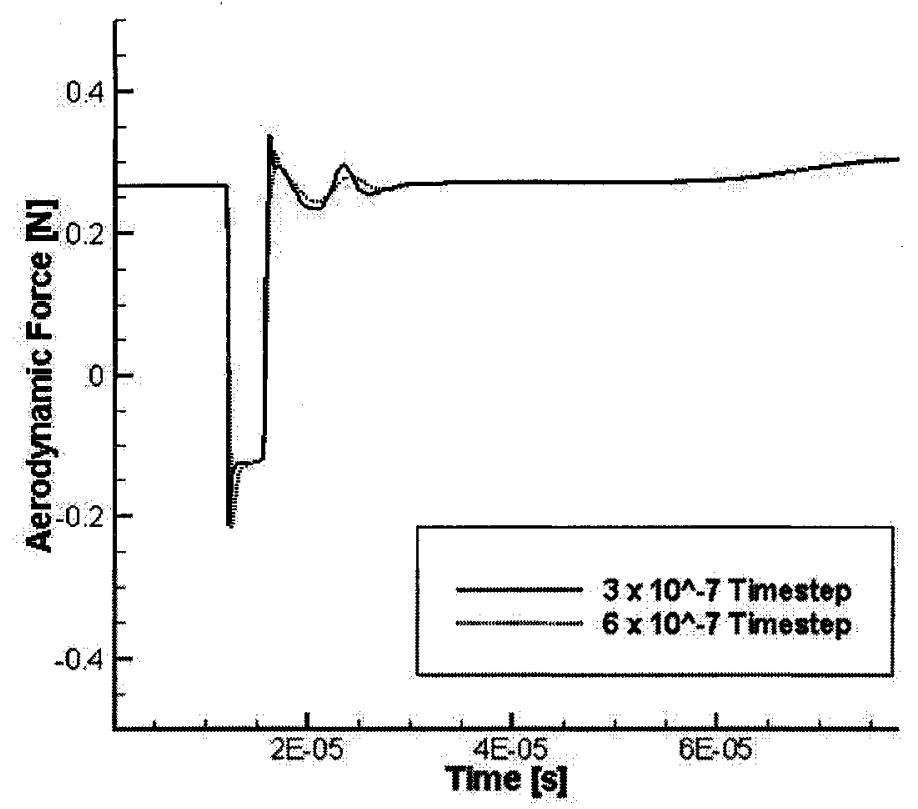

Figure 3.6: Timestep comparison, $F_{y_{0}}$ for mode 1

desired information.

It is important to remember what information was being sought through the CFD simulations. For each simulation it was desired to know what effect the fluid flow has on the blade. This effect can be quantified by the resultant aerodynamic force on the blade and resultant aerodynamic moment about the shear centre. With the goal of the post-processing defined, it remained to produce the desired data.

The CFD software, CFX-10.0, can display the output in several ways. It can be viewed in various ways in both the CFX solver and the CFX post-processor programs. While the solver program is useful to view graphical data over the entire time band, the post-processor is more useful for looking at flow field diagrams at a given timestep, or to produce animation files.

The data required was obtained using the CFX-Solve program to monitor the forces in the $y_{0}$ and $z_{0}$ directions, as well as the moment about the $x$-axis. These plots are shown in Figures 3.8, 3.9, and 3.10 for the first mode shape, first bending. 


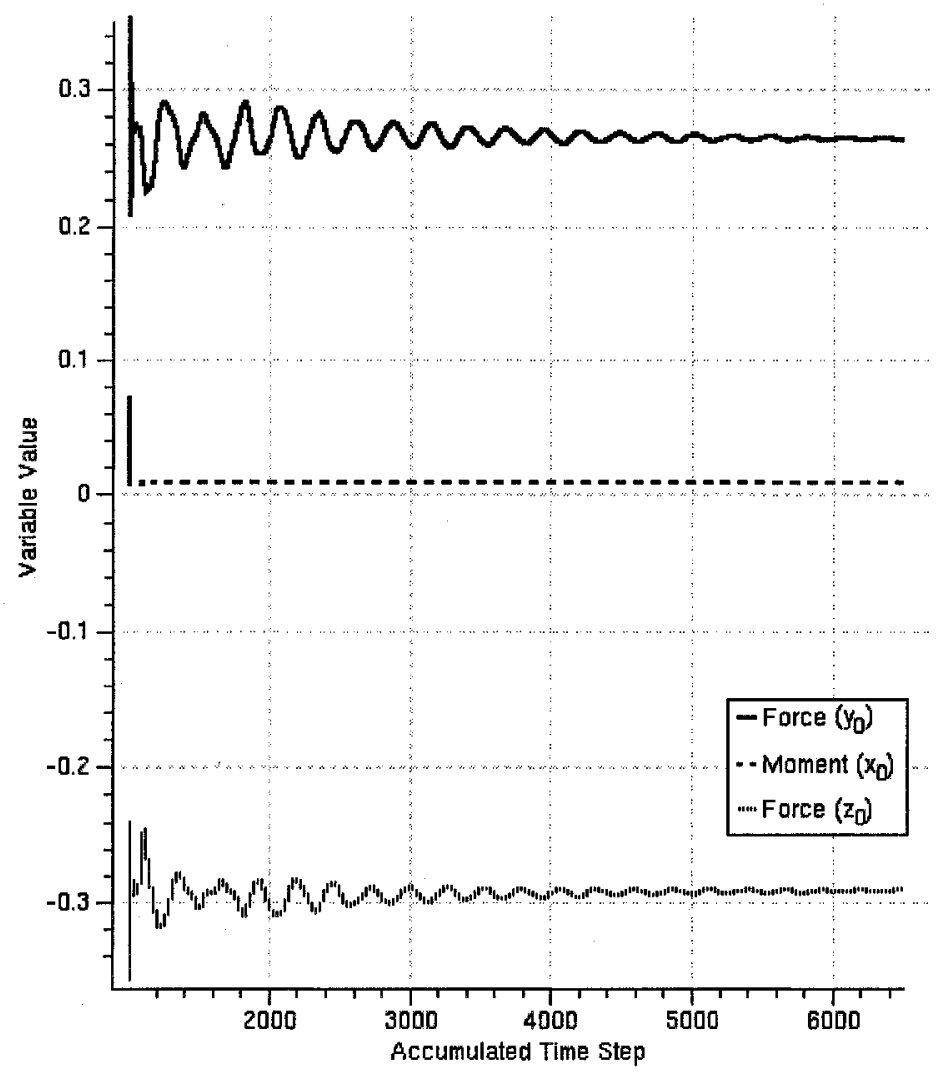

Figure 3.7: CFX-Solve plot showing the aerodynamic resultant forces and moment as they vary with the timestep (mode 1) 
Note that the initial blade displacement occurs at timestep 1010. Therefore, the first timestep considered for the response is timestep 1011.

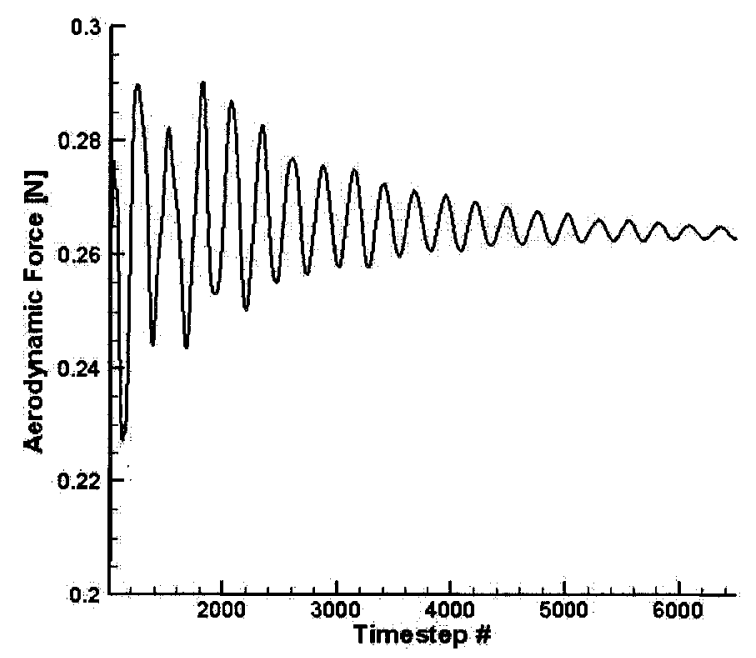

Figure 3.8: Aerodynamic force in the $y_{0}$ direction for mode 1

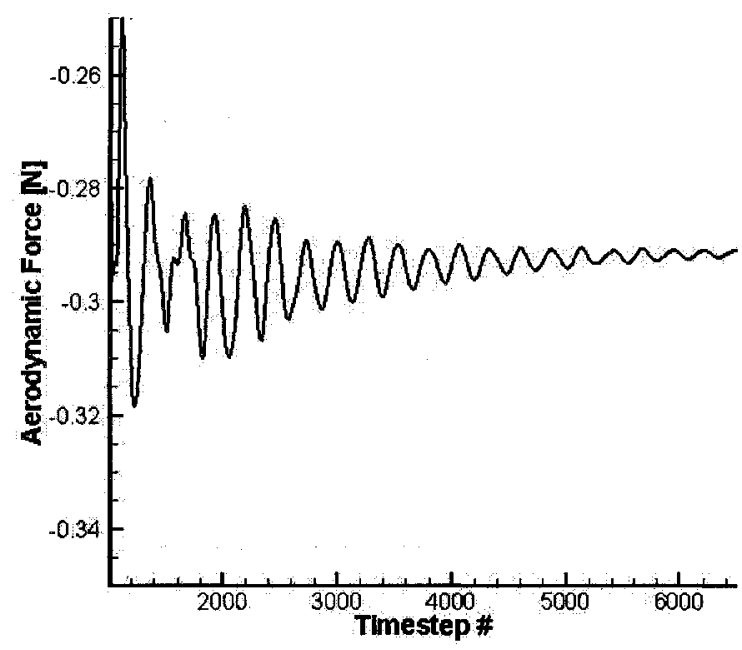

Figure 3.9: Aerodynamic force in the $z_{0}$ direction for mode 1

This output was helpful, but before it could be used it required a check to ensure that the data output was what was expected. This entails checking a single timestep pressure profile to determine the integrated loads over the blade and comparing it to 


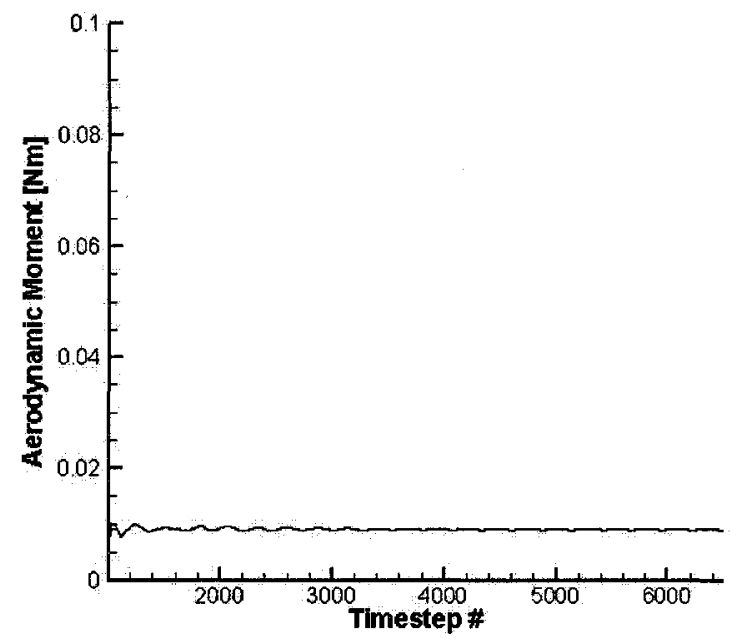

Figure 3.10: Aerodynamic moment about the $x_{0}$-axis for mode 1

the values produced in Figures 3.8 and 3.9. In a similar fashion the moment value was also checked.

The following section discusses the method used to check the solver output force data.

\subsubsection{Force Check}

The CFX-Solve program produced a plot similar to Figure 3.8 as a result of the transient simulation, due to the first natural mode of vibration (bending). This plot displays the force in the $y_{0}$ direction as it varies with the timestep. In order to be certain that this data represents the aerodynamics force on the blade in the $y_{0}$ direction, one must calculate the resultant force in the $y_{0}$ direction at a specific timestep, and compare it to the value shown in Figure 3.8. This has been done using pressure information from the CFX-Post program, as well as a Matlab code written expressly for this purpose.

The first step was to determine the pressure profile on the periphery of the blade at a given timestep. The pressure distribution along the spanwise direction ( $x$ direction) 
is roughly constant; therefore, the process could be simplified by looking only at the 2-D pressure profile. A 2-D section of the domain has a pressure profile as shown in Figure 3.11, for the final timestep, 6501 (which corresponds to a time value of $(6501-1010) \times\left(6 \times 10^{-7}\right)=3.294 \times 10^{-3}$ seconds after the blade perturbation $)$. Viewing only the pressure on the periphery of the blade produced Figure 3.12.

The data in Figure 3.12 can be represented graphically as pressure varying along the $y_{0}$ and $z_{0}$ directions, as shown in Figure 3.13.

Discretizing Figure 3.13 into points provides a series of data points describing the pressures at specific $\left(y_{0}, z_{0}\right)$ locations. This tabular data, listed in Appendix $\mathrm{B}$, is the starting point for determining the resultant aerodynamic forces on the blade at timestep 6501.

A Matlab m-file was written to transform the pressure values into resultant forces in the $y_{0}$ and $z_{0}$ directions. This m-file is shown in Appendix A; while the output data is shown in Appendix C.

The resultant forces in the $y_{0}$ and $z_{0}$ directions are $0.2630 \mathrm{~N}$ and $-0.2908 \mathrm{~N}$, respectively. These values are the same as those provided in the solver plots. Therefore, the data from the solver plots has been proven to provide the data expected.

A secondary check of the force data has also been used to verify the initial force value in the vertical $y_{0}$ direction. Aerodynamic theory provides the estimation shown in Equation 3.1.

$$
F_{y_{0}}=\dot{m}\left(v_{y_{0 i n}}-v_{y_{0 o u t}}\right)
$$

In Equation 3.1, the mass flow in the passage is shown as $\dot{m}$. The mass flow is calculated using the area of the inlet $\left(6.25 \times 10^{-6} \mathrm{~m}^{2}\right)$, the fluid velocity at the inlet $(168 \mathrm{~m} / \mathrm{s})$, and the density of air at the inlet $\left(0.527 \mathrm{~kg} / \mathrm{m}^{3}\right)$. This produced a mass flow of $5.335 \times 10^{-4} \mathrm{~kg} / \mathrm{s}$ at the inlet. The inlet velocity in the $y_{0}$ direction was calculated as a component of the magnitude $\left(168 \sin 14^{\circ}=40.6 \mathrm{~m} / \mathrm{s}\right)$. The outlet 


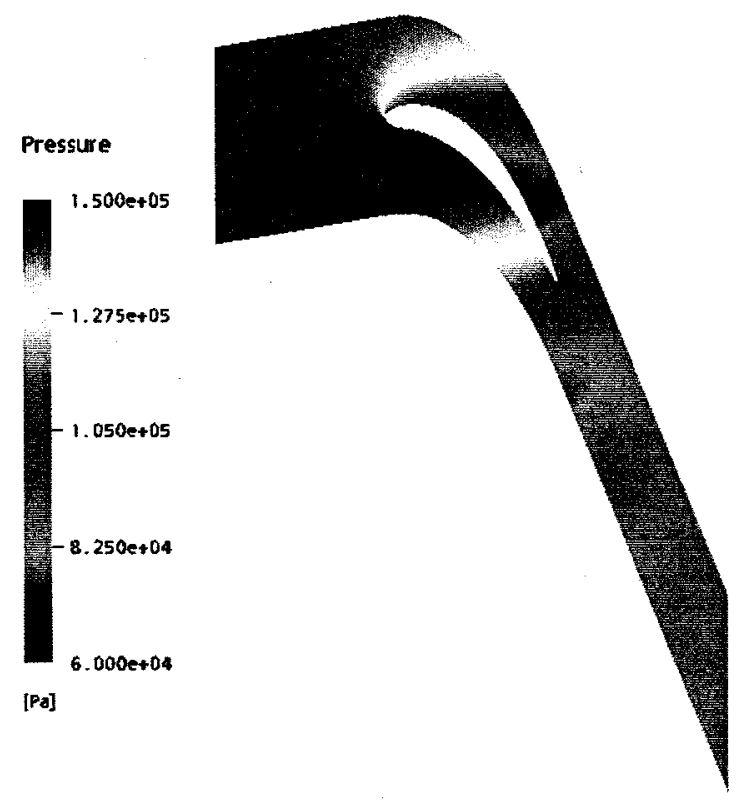

Figure 3.11: Pressure profile for mode 1 at timestep 6501

\section{Pressure}

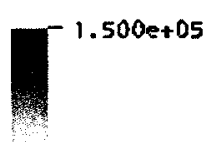

$-1.275 e+05$

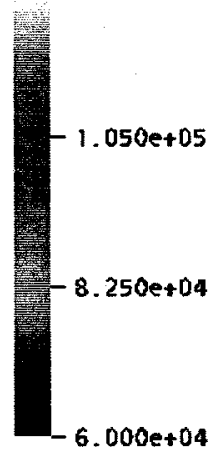

[Pa]

Figure 3.12: Pressure along blade periphery for mode 1 at timestep 6501 


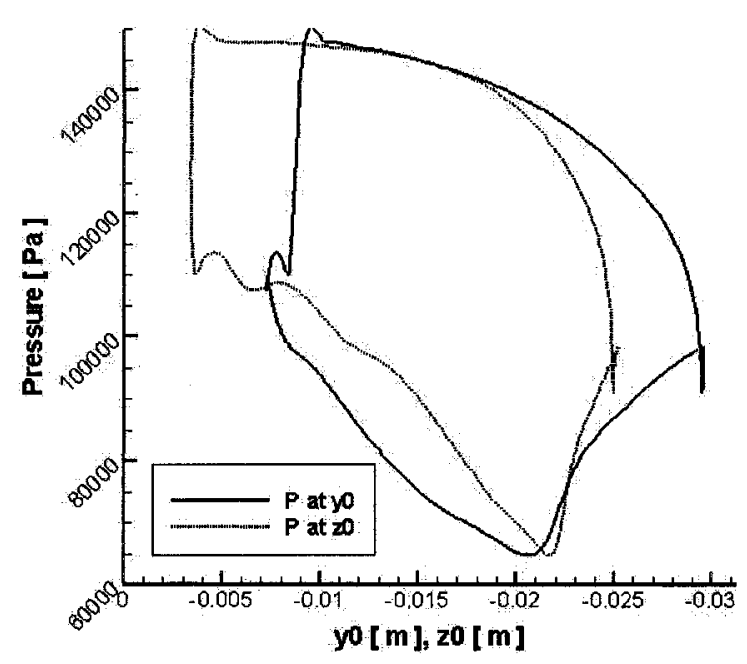

Figure 3.13: Pressure plot along blade periphery for mode 1 at timestep 6501

velocity in the $y_{0}$ direction was taken from CFX-Post as an average of the velocities at the outlet plane $(-446 \mathrm{~m} / \mathrm{s})$. Multiplying the mass flow rate by the difference between the inlet and outlet velocities (in the $y_{0}$ direction) produced a force of $0.269 \mathrm{~N}$. This value was compared to the value produced by the CFX solver $(0.266 \mathrm{~N})$. The values are close indicating that the CFD results are in the proper range.

With the forces squared away, it remained to determine the moment. This is calculated in the next section.

\subsubsection{Moment Check}

In the previous section, the resulting aerodynamic force of the blade was calculated at a specific timestep using the discretized pressure values along the periphery of the blade. In a similar fashion, the resultant moment can be found using the discrete forces acting around the periphery of the blade.

The Matlab $\mathrm{m}$-file written to determine the resultant forces in the $y_{0}$ and $z_{0}$ directions also contains code that calculates the resultant moment about the $x$-axis, Figure 4.5 illustrates orientation of this axis. The output for timestep 6501 produces a 
moment value of $0.0086 \mathrm{Nm}$ (for the thin slice; $0.00025 \mathrm{~m}$ thickness). This corresponds to the value found in the moment plot, Figure 3.10, exactly. This validates the output obtained from the CFX solver to be the resultant moment about the $x$-axis at the cartesian zero.

The resultant moment data has been verified. In the aeroelastic transfer function calculation, the COM and the SC have been assumed to be coincident. Therefore, the forces on the COM are not affected by the moment. As such there will be no more mention of the aerodynamic moment in this chapter.

The CFX program can output much data in several ways. The goal of the CFX simulations was to produce equations for the aerodynamic force in the $y_{0}$ and $z_{0}$ directions. The force data have been collected. It remained to fit equations to these data. The equations were fit using the least squares method in the next section.

\subsubsection{Curve Fitting}

In order to utilize the data collected from the CFX simulations, each force plot has been approximated by a curve. This section describes the curve fitting process using the method of least squares. Note that the following curve fitting process has been done using the aerodynamic response in the minor principal axis due to the excitation by the modes of structural vibration. This case is simply an example. The same process was used to determine to the forces in the $y_{0}$ and $z_{0}$ directions. Figure 3.14 illustrates the orientation of the axes.

The curve fitting for the first mode has been done using an oscillating decreasing function of the general form shown in Equation 3.2.

$$
f(t)=A e^{-B t} \cos (\omega t+C)+F
$$

where 


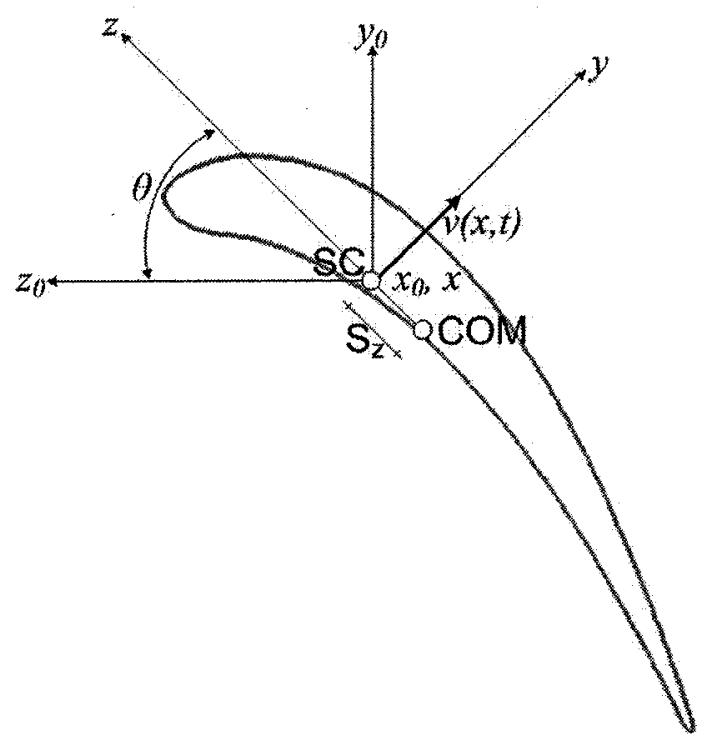

Figure 3.14: Blade cross-section with principal axes, $y$ and $z$

$A$-scales the amplitude of the oscillation

$B$-controls the rate of logarithmic decrement

$C$-defines the phase shift of the oscillation

$F$-vertically shifts the data

$t$-time

$\omega$-frequency of the oscillation

Note that the time, $t$, is used in this equation. It has therefore been beneficial to replace the timestep scale with the corresponding time value scale. It was also useful to zero the time at the first timestep after the blade perturbation. In this way, time zero occurred at the beginning of the useful output data. This new scale is shown in Figure 3.15 for the force in the $y$ direction.

The data shown in Figure 3.15 will be used to demonstrate the curve fitting process. In order to fit the data, the equation constants were found one by one, and 


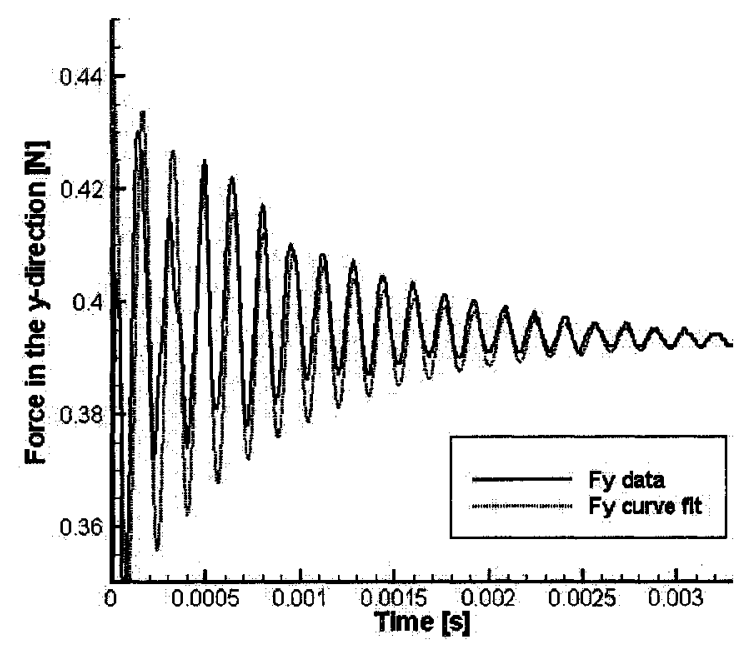

Figure 3.15: Mode 1 - force in the $y$ direction over time

optimized using the method of least squares. This method makes use of the squared sum of the residuals. It states that the best fit is obtained when the sum of the squared residuals is minimized.

The first constant to be defined was the frequency of oscillation in the data. This task was accomplished using Matlab and the built-in FFT (Fast Fourier Transform) function. The frequency of all the force data for all modes was found to be the same. The frequency value was $12,500 \pi \mathrm{rad} / \mathrm{s}$. The vertical shift of the curve is defined by the steady-state value of the oscillation. At the right side of the data plot the data is oscillating about $0.393 \mathrm{~N}$ (for mode 1). This value was assigned to the variable $F$.

The remaining constants were not as easily defined. The process to determine their optimal values was a combination between estimation and the least squares method. The remaining constants were given an initial value that provided a visually appealing match to the raw data. The initial values used for $F_{y}$ for mode 1 are shown in Table 3.2 and the curve fit using these values is shown in Figure 3.15. After the initial values were achieved, each constant was varied while monitoring the squared sum of the residuals. 
Table 3.2: Initial curve fit constants for $F_{y}$ (mode 1)

\begin{tabular}{|l|c|}
\hline$\cdot$ & Initial Value \\
\hline$A$ & 0.05 \\
$B$ & 1200 \\
$C$ & $-0.025(2 \pi)$ \\
\hline
\end{tabular}

There were three variables that needed optimization for mode 1. The phase shift was optimized first as it is independent of the amplitude and the rate of decrement. The other two variables have a dependent relationship; the larger the initial amplitude, the larger the decrement must be to satisfy the data (and vice versa). That said, the amplitude was optimized prior to the decrement, after which both were optimized a second time. The optimized values are shown in Table 3.3 and the curve fit with these values is shown in Figure 3.16.

Table 3.3: Final curve fit constants for $F_{y}$ (mode 1)

\begin{tabular}{|c|c|}
\hline & Optimized Value \\
\hline$A$ & 0.045 \\
$B$ & 1500 \\
$C$ & $0.04(2 \pi)$ \\
\hline
\end{tabular}

In this way the optimized curve fit was found for $F_{y}$. Unfortunately, the steadystate portion of the data is less accurate than the original visual fit. It is for this reason that the visual fits have been chosen over the least square fits.

At this stage a check was done to make certain that the simulations were in the linear range. The first mode of vibration was scaled to have half of the displacement and rotation as originally. Acquiring the solution and post-processing the data produced what is shown in Figure 3.17. The equation that fits this data has the constants shown in Table 3.4.

The differences between the original state and the halved amplitude state are 


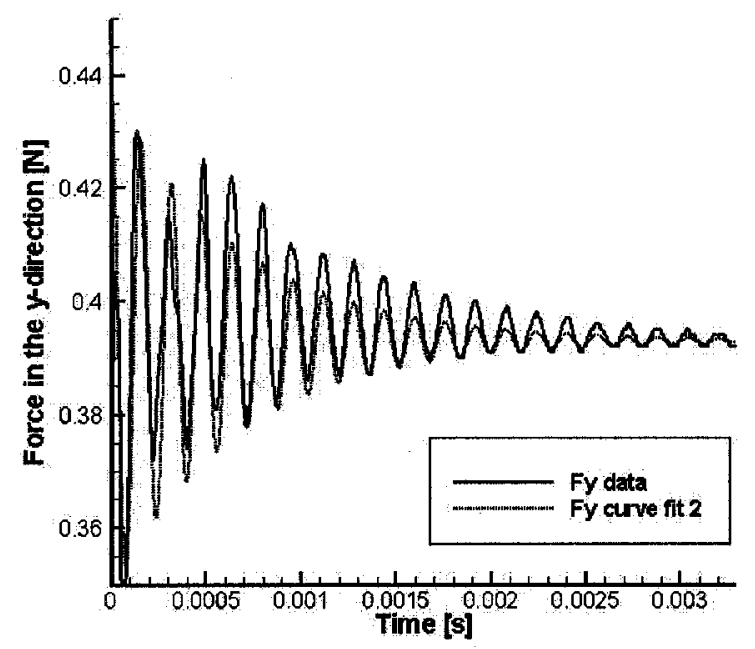

Figure 3.16: Mode 1 - force in the $y$ direction over time (optimized curve fit)

Table 3.4: Curve fit constants for $F_{y}$ (mode 1 - half amplitude)

\begin{tabular}{|c|c|}
\hline & Value \\
\hline$A$ & 0.025 \\
$B$ & 1200 \\
$C$ & $-0.025(2 \pi)$ \\
$F$ & 0.3955 \\
$\omega$ & $12,500 \pi$ \\
\hline
\end{tabular}

the variables $A$ and $F$. If a linear relationship is to exist, it is expected that if the amplitude of the displacement changes, the amplitude of the signal will be scaled by the same factor. This is true for the amplitude of the output signals, $A$ values. The second change was in the $F$ variable, which represents the value about which the signal is oscillating. If the angular orientation of the blade is at all changed, it is expected that this value will also change. For the force data, the $F$ represents the average force value at the end of the signal. The undisplaced blade data showed a steady-state force in the $y$ direction of $0.397 \mathrm{~N}$. This indicates that a halved amplitude simulation would be expected to have a $0.395 \mathrm{~N}$ average force value at the end of the signal (halfway 


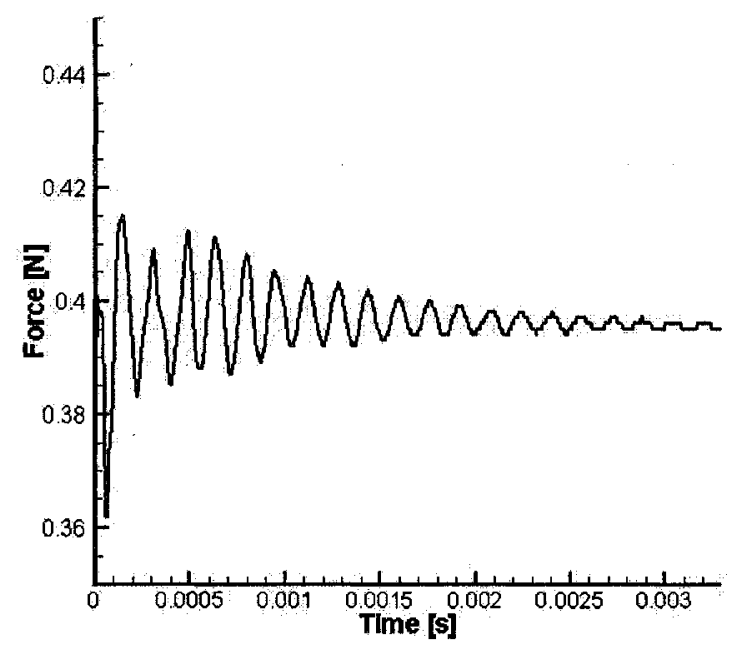

Figure 3.17: Mode 1 (half amplitude) - force in the $y$ direction over time

between $0.397 \mathrm{~N}$ and $0.393 \mathrm{~N}$ ). The actual value is $0.3955 \mathrm{~N}$ for the halved amplitude simulation. This indicates that the input and output do not completely follow the linear rules. However, this was discovered after most of the work was complete. As a result, for simplicity the data has been treated as though it were within the linear range.

Returning to the curve fitting process, the second and third modes produced results that were slightly more complicated than the first mode. This is due to the increased amount of rotation in the blade displacement. As a result the steady-state value of the forces changed significantly from the initial. In order to account for this change the general equation for the curve fit needed an extra term, as shown in Equation 3.3.

$$
f(t)=A e^{-B t} \cos (\omega t+C)+D \arctan (E t)+F
$$

In this more complicated general equation, the arctan function accounts for the change in steady-state value from the initial to the final state.

The curve fitting process shown in this section has been used in the next section 
order to provide the aerodynamic force responses in the $y_{0}$ and $z_{0}$.

\subsection{Curve Fitting in the $y_{0}$ and $z_{0}$ directions}

This research couples the ANSYS simulation output directly to the CFX output. In order to couple these data properly, the aerodynamic forces must be expressed in the same coordinate system as the ANSYS output. This means expressing the aerodynamic forces in the $y_{0}$ and $z_{0}$ axes. This section displays the data in the required form.

The aerodynamic force data in the $y_{0}$ and $z_{0}$ directions was captured by the CFXSolve program. This data is shown, for mode 1, in Figures 3.8, and 3.9. The curve fitting for the force data was done as described in Section 3.4.3. The equations for these curve fits are shown in Equation 3.4. The curve fit plots for each of the cases are shown in Appendix D.

$$
\begin{aligned}
& M 1 F_{y_{0}}(t)=0.035 e^{-1100 t} \cos (12,500 \pi t+0.004(2 \pi))+0.26425 \\
& M 2 F_{y_{0}}(t)=0.050 e^{-1000 t} \cos (12,500 \pi t)-0.045 \arctan (1000 t)+0.294 \\
& M 3 F_{y_{0}}(t)=0.060 e^{-1200 t} \cos (12,500 \pi t+0.550(2 \pi))+0.03 \arctan (1000 t)+0.252 \\
& M 1 F_{z_{0}}(t)=0.025 e^{-1200 t} \cos (12,500 \pi t+0.55(2 \pi))+0.0055 \arctan (1000 t)-0.2986 \\
& M 2 F_{z_{0}}(t)=0.030 e^{-950 t} \cos (12,500 \pi t+0.50(2 \pi))+0.045 \arctan (500 t)-0.2925 \\
& M 3 F_{z_{0}}(t)=0.030 e^{-1050 t} \cos (12,500 \pi t+0.05(2 \pi))-0.036 \arctan (750 t)-0.29425
\end{aligned}
$$

The aerodynamic information required has been collected and post-processed. The next chapter derives the equations of motion for the rotating beam system. This 
derivation is intended as an introduction for the modal analysis which follows. 


\section{Chapter 4}

\section{STRUCTURAL FIELD EQUATION MODEL DERIVATION}

The purpose of this chapter is to produce the structural field equations and the corresponding boundary conditions that describe a turbine blade attached to a rigid rotating hub. These equations have been produced to demonstrate the theoretical basis of the proposed methodology.

The blade was assumed to deform only in bending and twist (about the shear centre). The energy method was used to define the potential and kinetic energy terms in the system, as well as the non-conservative force term. Hamilton's equation was used to derive the field equations and the boundary conditions. The chapter concludes with brief explanation of solution techniques.

The rotating blade system that was to be analyzed consisted of an Euler-Bernoulli beam cantilevered to a rigid rotating hub. This system is shown in Figure 4.1 where the $X, Y$ and $Z$ axis are stationary, and the variable $R$ denotes the hub radius. Rotating axes were denoted as $x_{0}, y_{0}$, and $z_{0}$; these rotated with the hub at $\dot{\alpha}, 2880$ $\mathrm{rad} / \mathrm{s}(27,500 \mathrm{rpm})[1]$, and were fixed at the root of the blade at the point defined by the local shear centre $(S C)$.

In this work, the direction of the bending was assumed to be in the minor principal 


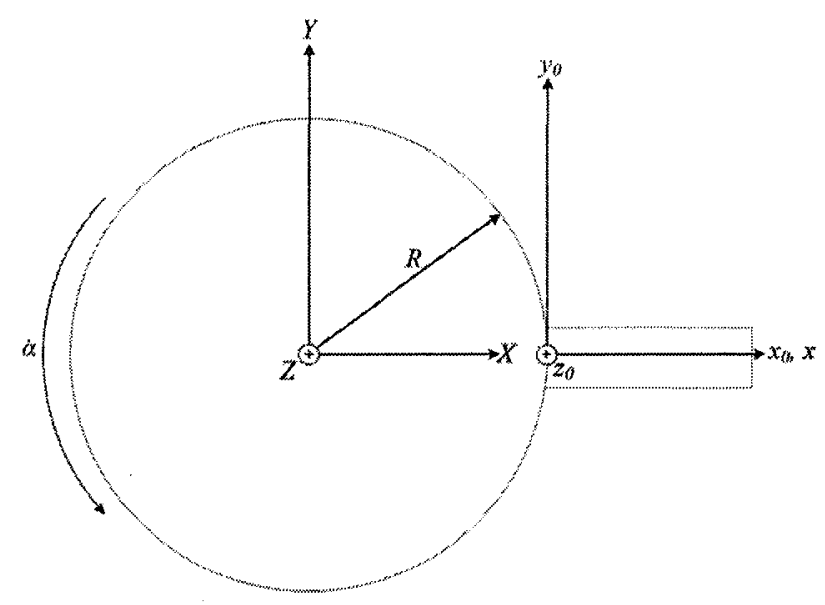

Figure 4.1: Rotating hub-beam system as seen from the positive $Z$-axis

axis, which lies at an angle, $\theta$, from the $y_{0}$-axis. This assumption was made as the minor principal axis has the least amount of resistance to bending, and thus will experience the most deflection. A third set of axes were easily defined to describe the principal axes, $x, y$ and $z$. The $y$ - and $z$-axes remain in the same plane defined by the $y_{0}$ - and $z_{0}$-axes, and have the same origin. Therefore, the $x$ - and $x_{0}$-axes are coincident. The new $z$-axis is at an angle to the rotation axis, $Z$, as seen in Figure 4.2 .

The beam was considered to be linearly elastic with uniform cross-section from root to tip. The cross-section of the blade is shown in Figure 4.3. It was assumed that the beam properties were isotropic and that all deflections were small. The axial deformation was taken to be negligible. In fact only two deformation directions were taken into account; bending, $v(x, t)$ (which occurred in the rotating $y$ direction) and twisting, $\psi(x, t)$ (which occurred about the $x$-axis).

The hub rotates about the $Z$-axis, the blade was fixed to the hub, and the rotating axes remained attached at the blade root at the shear centre of the cross-section. The rotating, $x_{0}$-axis was directed along the undeformed blade span. The rotating $y_{0}$-axis 


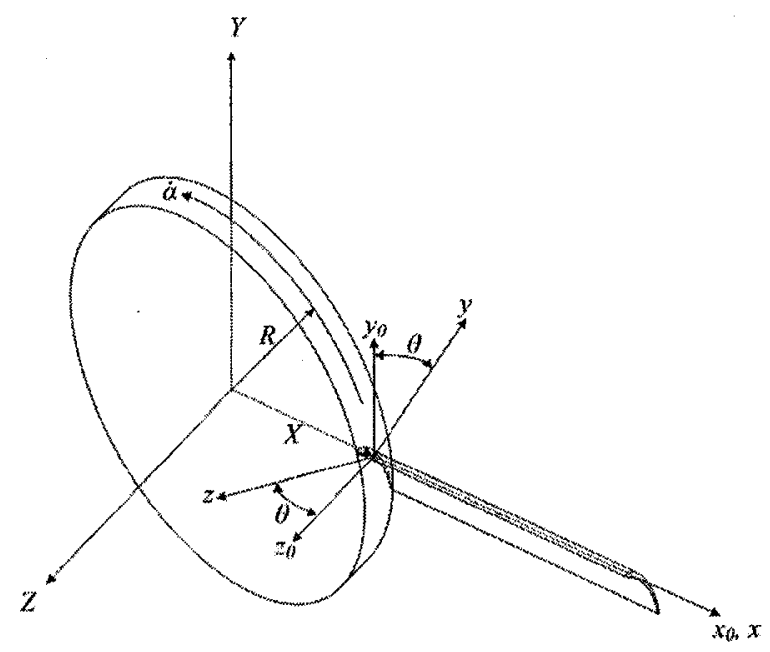

Figure 4.2: Isometric view of rotating hub-beam system

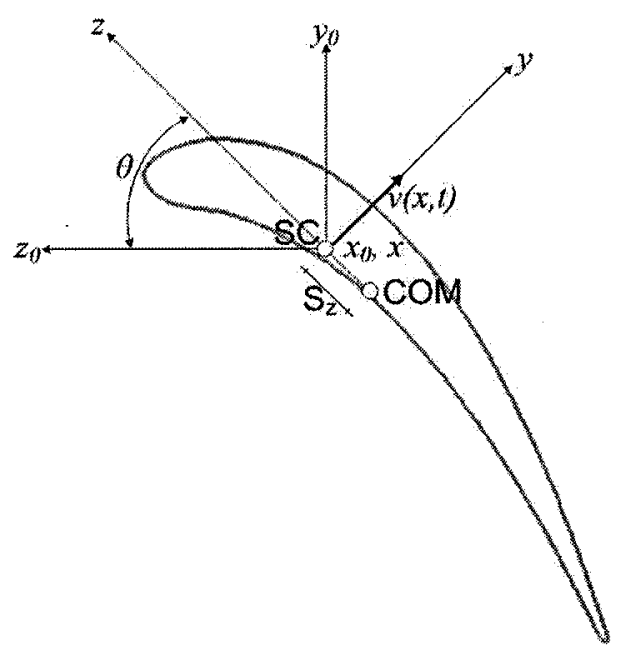

Figure 4.3: Blade cross-section as seen from the positive $x_{0}$-axis 
lies tangent to the hub periphery at the connection point. The rotating $z_{0}$-axis, shown in Figure 4.1, is perpendicular to both the $x_{0}$ and $y_{0}$ direction and as such, points directly out of the page.

Figure 4.4 illustrates the blade cross-section with the bending and twist directions shown; bending along the $y$-axis, and twisting about the $x$-axis.

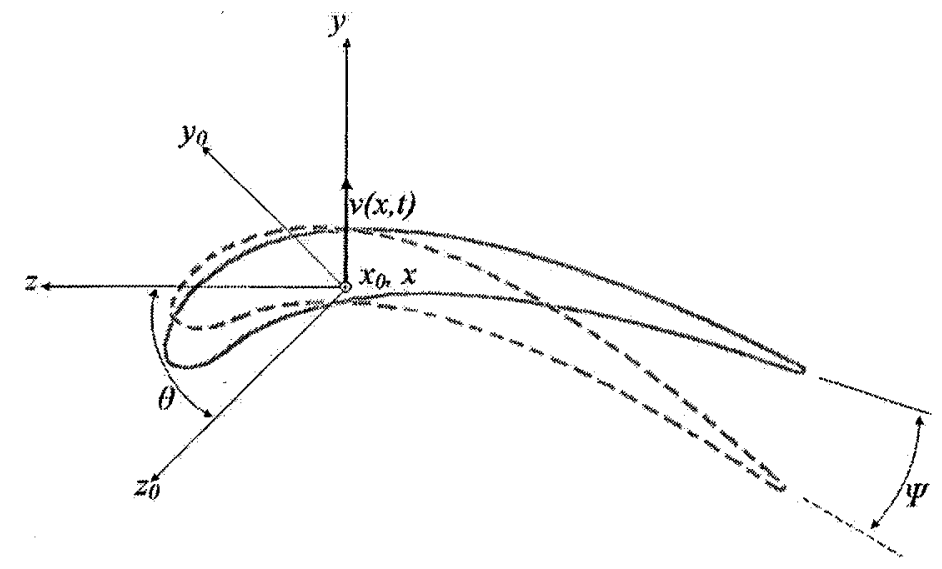

Figure 4.4: Blade cross-section with twist about the shear centre

The turbine consisted of a disk, the rotation of which was caused by the aerodynamic forces working on the blade. As such, the torque at the hub was a resisting force, acting in the opposite direction of the blade motion. This resistance was caused by the attached turbine components; such as the compressor disk. The resisting torque, $\tau(t)$, was treated as an unknown which acts about the non-rotating $Z$-axis.

Figure 4.4 illustrates the $y-z$ plane. The beam cross-section is an airfoil with the undeformed cross-section appearing in bold lines. The dashed airfoil periphery denotes a twist of the blade cross-section about the shear centre $(S C)$. The twist angle was measured by the variable $\psi(x, t)$.

The shear centre was assumed to be upstream of the centre of mass and that both points were located on the $z$-axis. The distance between the centre of mass and the shear centre was denoted by the variable $S_{z}$. Although the analysis in the 
next chapter assumes no shear centre offset, the equations have been derived for the general case in which there may be a non-zero offset value.

The derivation of the system equations for the simplified problem began with defining the kinetic and potential energy equations for a beam attached to a rotating hub. Hamilton's Principle was used to map the energy of the system into governing equations of motion, and the corresponding boundary conditions.

\subsection{Kinetic Energy}

The system possesses kinetic energy, $T$, due to three contributors; blade rotation about the $Z$-axis $(b r)$, blade twisting about the shear centre $(b t)$ and hub rotation about the $Z$-axis $(h r)$. The contribution made by the blade rotation was derived first.

The blade rotates about the hub centre at a constant rotational speed. As the blade bends in the $X-Y$ plane the local rotational speeds at each spanwise location vary. It was therefore necessary to consider these variations in the kinetic energy equation. An integral over the length of the blade facilitated this procedure using a position vector, $\vec{r}(x, t)$, to denote the distance from the hub centre to the centre of mass at any spanwise location on the blade.

To aid in visualizing the position vector, a test point $\mathrm{P}$ was defined. It was situated at the centre of mass at a distance $x$ from the $y$-axis; it deforms to point $\mathrm{P}^{\prime}$ causing a deflection from the $x$-axis of $v(x, t)$, and a twist about the shear centre of $\psi(x, t)$. The position vector, $\vec{r}(x, t)$, was defined as shown in Figure 4.5.

Although it is difficult to see in Figure 4.5, the position vector, $\vec{r}(x, t)$, points to the centre of mass of the cross-section. The bending deflection, $v(x, t)$, is defined between the shear centers of the undeformed and deformed states. Figure 4.6 is a magnified view of Figure 4.5 to facilitate visualization. In order to fully define the position vector, the twist of the blade must be considered. 


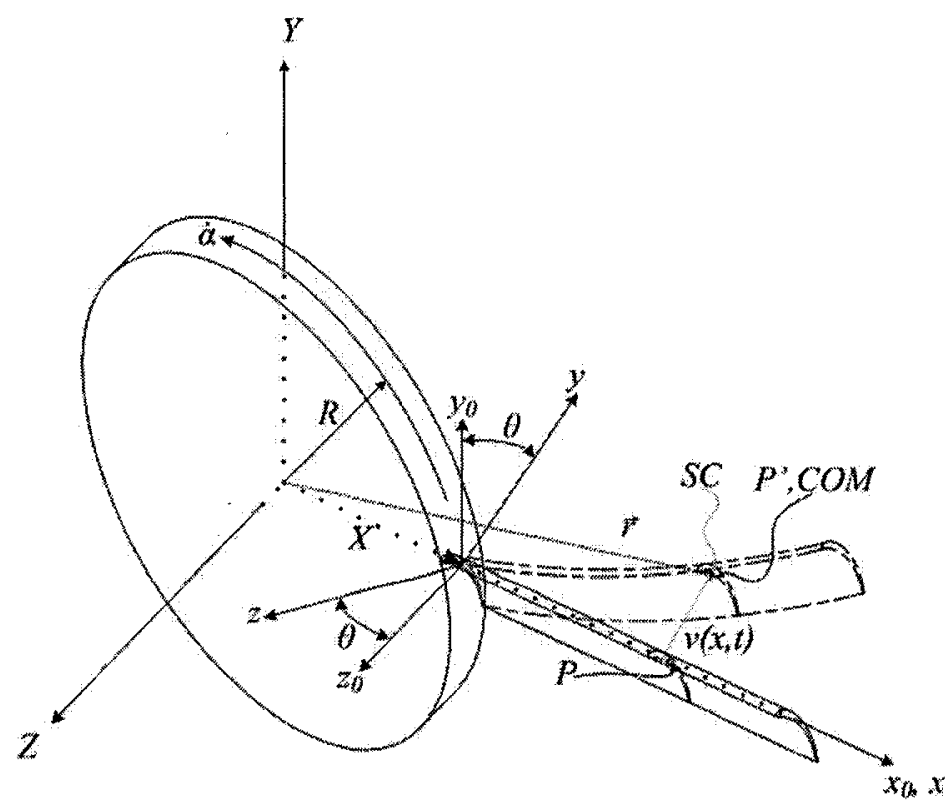

Figure 4.5: Position vector, $\vec{r}(x, t)$

The kinetic energy due to the blade rotation, about the $Z$-axis, is defined by Equation 4.1.

$$
T_{b r}=\frac{1}{2} \rho A_{b} \int_{0}^{L} \dot{\vec{r}}(x, t) \cdot \dot{\vec{r}}(x, t) d x
$$

Note that in Equation 4.1 the dot symbol, (), denotes a derivative with respect to time, $t$. The position vector, $\vec{r}(x, t)$, can be defined as shown in Equation 4.2.

$$
\vec{r}(x, t)=\{R+x\} \vec{i}+\left\{v(x, t)+S_{z} \sin \psi(x, t)\right\} \vec{j}+\left\{-S_{z} \cos \psi(x, t)\right\} \vec{k}
$$

In the above equation the unit vectors in the $x, y$, and $z$ directions are represented by $\vec{i}, \vec{j}$, and $\vec{k}$, respectively. It was noted that the angle $\psi$ is small, and thus a small angle approximation was used to reduce Equation 4.2 to Equation 4.3.

$$
\vec{r}(x, t)=\{R+x\} \vec{i}+\left\{v(x, t)+S_{z} \psi(x, t)\right\} \vec{j}+\left\{-S_{z}\right\} \vec{k}
$$




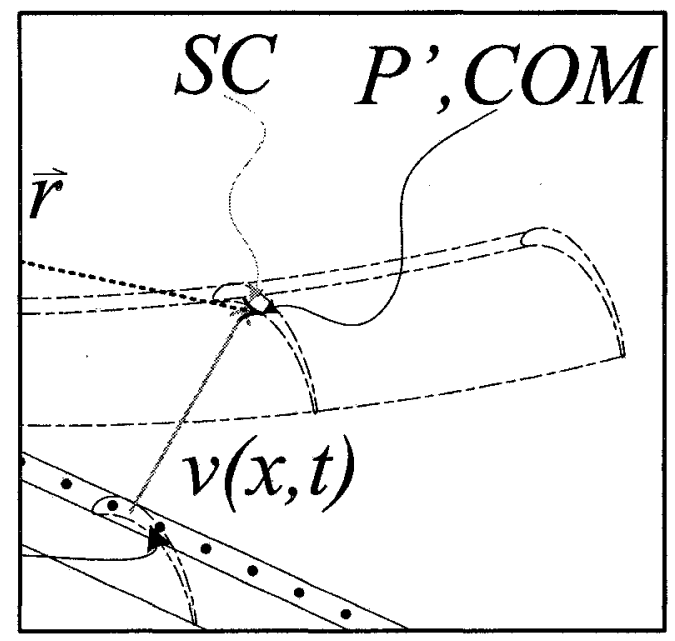

Figure 4.6: Position vector, $\vec{r}(x, t)$

It can be seen in Equation 4.3 that the shear centre offset, $S_{z}$, and the twist angle, $\psi$, affect the $y$ and $z$ values of the position vector. The derivation of Equation 4.3 with respect to time produces the following.

$$
\begin{aligned}
\dot{\vec{r}}(x, t)= & -\left\{\left(v(x, t)+S_{z} \psi(x, t)\right) \cos \theta-S_{z} \sin \theta\right\} \dot{\alpha} \vec{i} \\
& +\left\{(R+x) \dot{\alpha} \cos \theta+\dot{v}(x, t)+S_{z} \dot{\psi}(x, t)\right\} \vec{j} \\
& +\{(R+x) \dot{\alpha} \sin \theta\} \vec{k}
\end{aligned}
$$

Using the relation shown in Equation 4.4, the kinetic energy due to the blade rotation could be expressed as follows, in Equation 4.5.

$$
\begin{aligned}
T_{b r}(t)= & \frac{1}{2} \rho A_{b} \int_{0}^{L}\left[\left\{\left(v(x, t)+S_{z} \psi(x, t)\right) \cos \theta-S_{z} \sin \theta\right\}^{2} \dot{\alpha}^{2}\right. \\
& +\left\{(R+x) \dot{\alpha} \cos \theta+\dot{v}(x, t)+S_{z} \dot{\psi}(x, t)\right\}^{2} \\
& \left.+\{(R+x) \dot{\alpha} \sin \theta\}^{2}\right] d x
\end{aligned}
$$

The blade twisting also adds kinetic energy to the system. The equation for this term is given in [17]. Rewriting this equation to reflect the variables in the current 
work produced Equation 4.6. The $J_{S C}$ term represents the polar moment of inertia of the blade cross-section about the shear centre.

$$
T_{b t}(t)=\frac{1}{2} \rho J_{S C} \int_{0}^{L} \dot{\psi}^{2}(x, t) d x
$$

The final kinetic energy term to be defined was that caused by the spinning of the rigid hub. This was a simple equation, shown by Yang and Chen [5].

$$
T_{h r}=\frac{1}{2} I_{h} \dot{\alpha}^{2}
$$

Therefore, the full kinetic energy of the system could be written as shown in Equation 4.8.

$$
\begin{aligned}
T(t)= & \frac{1}{2} \rho A_{b} \int_{0}^{L}\left[\left\{\left(v(x, t)+S_{z} \psi(x, t)\right) \cos \theta-S_{z} \sin \theta\right\}^{2} \dot{\alpha}^{2}\right. \\
& +\left\{(R+x) \dot{\alpha} \cos \theta+\dot{v}(x, t)+S_{z} \dot{\psi}(x, t)\right\}^{2} \\
& \left.+\{(R+x) \dot{\alpha} \sin \theta\}^{2}\right] d x \\
& +\frac{1}{2} \rho J_{S C} \int_{0}^{L} \dot{\psi}^{2}(x, t) d x+\frac{1}{2} I_{h} \dot{\alpha}^{2}
\end{aligned}
$$

With the kinetic energy equation defined, the potential energy of the system was derived next.

\subsection{Potential Energy}

Potential energy, $V$, arises from the elastic potential of the system and also from the centrifugal stiffening force $(c s f)$. There were two elastic potential terms; one for the beam bending and another for the beam twist. These two terms are shown below; indicated by the subscript $e p$. 


$$
V_{e p}(t)=\frac{1}{2} E_{b} I_{S C} \int_{0}^{L} v^{\prime \prime 2}(x, t) d x+\frac{1}{2} G J_{S C} \int_{0}^{L} \psi^{\prime 2}(x, t) d x
$$

In Equation 4.9 the prime symbol, ('), denotes a derivative with respect to length, $x$.

There is potential energy associated with the centrifugal force. As discussed by Yang and Chen [5], the stiffening of the blade adds strain energy to the system, and must be considered in the potential energy calculation. The general equation for the potential energy due to centrifugal force is shown in Equation 4.10. Note that the energy is applied negatively, as it is an energy term due to an applied load, [18].

$$
V_{c s f}(t)=-\frac{1}{2} \int_{0}^{L} F_{c}(x) v^{2}(x, t) d x
$$

The centrifugal force, $F_{c}(x)$, was easily defined as in Yang and Chen [5]. Note that $\zeta$ was a dummy variable used for integration. Equation 4.11 displays the centrifugal force equation. The former is the general form, while the latter was obtained by taking the integration thereby eliminating the $\zeta$ variable.

$$
F_{c}(x)=\rho A_{b} \int_{x}^{L} \dot{\alpha}^{2}[R+\zeta] d \zeta=\rho A_{b} \dot{\alpha}^{2}\left[R(L-x)+\frac{1}{2}\left(L^{2}-x^{2}\right)\right]
$$

Combining all of the individual components that define the potential energy provided the following equation:

$$
\begin{aligned}
V(t)= & \frac{1}{2} E_{b} I_{S C} \int_{0}^{L} v^{\prime \prime 2}(x, t) d x+\frac{1}{2} G J_{S C} \int_{0}^{L} \psi^{\prime 2}(x, t) d x-\frac{1}{2} \int_{0}^{L} \rho A_{b} \dot{\alpha}^{2}[R(L-x) \\
& \left.+\frac{1}{2}\left(L^{2}-x^{2}\right)\right] v^{\prime 2}(x, t) d x
\end{aligned}
$$

As the potential and kinetic energies in the rotating beam system have now been defined, at this stage Hamilton's Principle can be used to determine the governing 
equations of motion as well as the corresponding boundary conditions. This process is discussed in the following section.

\subsection{Equations of Motion}

The equations of motion for the rotating beam system were determined using Hamilton's Principle, and then simplified using the method of separation of variables. Hamilton's Principle is stated as shown in Equation 4.13, [19]. This equation states that the time integral of the variation of the kinetic energy minus the variation of the potential energy plus the variation of the work produced by the non-conservative forces produces a value of zero.

$$
\int_{t 1}^{t 2}(\delta T(t)-\delta V(t)+\delta W(t)) d t=0
$$

The kinetic and potential energies have been defined in the previous sections. Taking the variation of these equations produces the following where the explicit functional $(x, t)$ has been omitted for simplicity. Note also that the rotational speed of the turbine, $\dot{\alpha}$, is constant (steady-state operation) and therefore the terms that include rotational acceleration, $\ddot{\alpha}$, have been neglected.

$$
\begin{aligned}
\delta T(t)= & -\int_{0}^{L} \rho A_{b}\left\{\left[\dot{\alpha}^{2} \cos ^{2} \theta\left(v+S_{z} \psi\right)-S_{z} \ddot{\psi}-\ddot{v}\right] \delta v\right. \\
& +\left[\dot{\alpha}^{2} S_{z} \cos ^{2} \theta\left(v+S_{z} \psi\right)-S_{z}^{2}\left(\ddot{\psi}-S_{z} \ddot{v}\right] \delta \psi\right. \\
& \left.+\left[-(R+x) \cos \theta\left(\ddot{v}+S_{z} \ddot{\psi}\right)-2 \dot{\alpha} \cos ^{2} \theta\left(v \dot{v}+S_{z} \dot{v} \psi+S_{z} v \dot{\psi}+S_{z}^{2} \psi \dot{\psi}\right)\right] \delta \alpha\right\} d x \\
& -\int_{0}^{L} \rho J_{S C} \ddot{\psi} \delta \psi d x
\end{aligned}
$$




$$
\begin{aligned}
\delta V(t)= & \left.E_{b} I_{S C} v^{\prime \prime} \delta v^{\prime}\right|_{0} ^{L}-\left.E_{b} I_{S C} v^{\prime \prime \prime} \delta v\right|_{0} ^{L}+\int_{0}^{L} E_{b} I_{S C} v^{\prime \prime \prime \prime} \delta v d x \\
& +\int_{0}^{L} \rho A_{b}\left[R(L-x)+\frac{1}{2}\left(L^{2}-x^{2}\right)\right]\left(2 \dot{\alpha} v^{\prime} \dot{v}^{\prime}\right) \delta \alpha d x \\
& -\left.\rho A_{b} \dot{\alpha}^{2}\left[R(L-x)+\frac{1}{2}\left(L^{2}-x^{2}\right)\right] v^{\prime} \delta v\right|_{0} ^{L} \\
& +\int_{0}^{L} \rho A_{b} \dot{\alpha}^{2}\left[v^{\prime \prime}\left[R(L-x)+\frac{1}{2}\left(L^{2}-x^{2}\right)\right]-v^{\prime}(R+x)\right] \delta v d x \\
& +\left.G J_{S C} \psi^{\prime} \delta \psi\right|_{0} ^{L}-\int_{0}^{L} G J_{S C} \psi^{\prime \prime} \delta \psi d x
\end{aligned}
$$

The virtual work due to the non-conservative forces were determined in order to possess all terms present in Hamilton's Principle. There was virtual work associated with each degree of freedom in the system; rotation about the hub centre (which affects the torque about the hub centre, $\tau(t)$ ), bending deflection (which affects the aerodynamic force, $F_{\infty}(t)$ ), and blade twist (which affects the aerodynamic moment, $\left.M_{\infty}(t)\right)$. Note that the work done by the torque is negative as it resists the motion.

$$
\delta W(t)=-\tau(t) \delta \alpha+F_{\infty}(t) \delta v+M_{\infty}(t) \delta \psi
$$

Applying Hamilton's Principle, and considering that $\delta v, \delta \alpha$ and $\delta \psi$ are arbitrary independent variations, the following equations of motion were found. 


$$
\begin{aligned}
-F_{\infty}(t)= & \int_{0}^{L}\left\{\rho A _ { b } \left(\dot{\alpha}^{2} \cos ^{2} \theta\left(v+S_{z} \psi\right)-S_{z} \ddot{\psi}-\ddot{v}\right.\right. \\
& \left.\left.-\dot{\alpha}^{2}\left[v^{\prime \prime}\left[R(L-x)+\frac{1}{2}\left(L^{2}-x^{2}\right)\right]-v^{\prime}(R+x)\right]\right)-E_{b} I_{S C} v^{\prime \prime \prime \prime}\right\} d x \\
\tau(t)= & \int_{0}^{L} \rho A_{b}\left\{-(R+x) \cos \theta\left(\ddot{v}+S_{z} \ddot{\psi}\right)-2 \dot{\alpha} \cos ^{2} \theta\left(v \dot{v}+S_{z} \dot{v} \psi+S_{z} v \dot{\psi}+S_{z}^{2} \psi \dot{\psi}\right)\right. \\
& \left.-2\left[R(L-x)+\frac{1}{2}\left(L^{2}-x^{2}\right)\right] \dot{\alpha} v^{\prime} \dot{v}^{\prime}\right\} d x \\
-M_{\infty}(t)= & \int_{0}^{L}\left\{\rho A_{b}\left(\dot{\alpha}^{2} S_{z} \cos ^{2} \theta\left(v+S_{z} \psi\right)-S_{z}^{2}\left(\ddot{\psi}+\psi \dot{\psi}^{2}+\psi^{2} \dot{\psi}\right)-S_{z} \ddot{v}\right)\right. \\
& \left.-\rho J_{S C} \ddot{\psi}+G J_{S C} \psi^{\prime \prime}\right\} d x
\end{aligned}
$$

Linearizing the equations of motion produces the following.

$$
\begin{aligned}
-F_{\infty}(t)= & \int_{0}^{L}\left\{\rho A _ { b } \left(\dot{\alpha}^{2} \cos ^{2} \theta\left(v+S_{z} \psi\right)-S_{z} \ddot{\psi}-\ddot{v}\right.\right. \\
& \left.\left.-\dot{\alpha}^{2}\left[v^{\prime \prime}\left[R(L-x)+\frac{1}{2}\left(L^{2}-x^{2}\right)\right]-v^{\prime}(R+x)\right]\right)-E_{b} I_{S C} v^{\prime \prime \prime \prime}\right\} d x \\
\tau(t)= & \int_{0}^{L} \rho A_{b}\left\{-(R+x) \cos \theta\left(\ddot{v}+S_{z} \ddot{\psi}\right)\right\} d x \\
-M_{\infty}(t)= & \int_{0}^{L}\left\{\rho A_{b}\left(\dot{\alpha}^{2} S_{z} \cos ^{2} \theta\left(v+S_{z} \psi\right)-S_{z} \ddot{v}\right)-\rho J_{S C} \ddot{\psi}+G J_{S C} \psi^{\prime \prime}\right\} d x
\end{aligned}
$$

The integration in the field equations was eliminated by derivating both sides of the equations with respect to the spatial variable. 


$$
\begin{aligned}
-\frac{d}{d x} F_{\infty}(t)= & \rho A_{b}\left(\dot{\alpha}^{2} \cos ^{2} \theta\left(v+S_{z} \psi\right)-S_{z} \ddot{\psi}-\ddot{v}\right. \\
& \left.-\dot{\alpha}^{2}\left[v^{\prime \prime}\left[R(L-x)+\frac{1}{2}\left(L^{2}-x^{2}\right)\right]-v^{\prime}(R+x)\right]\right)-E_{b} I_{S C} v^{\prime \prime \prime \prime} \\
\frac{d}{d x} \tau(t)= & \rho A_{b}\left\{-(R+x) \cos \theta\left(\ddot{v}+S_{z} \ddot{\psi}\right)\right\} \\
-\frac{d}{d x} M_{\infty}(t)= & \rho A_{b}\left(\dot{\alpha}^{2} S_{z} \cos ^{2} \theta\left(v+S_{z} \psi\right)-S_{z} \ddot{v}\right)-\rho J_{S C} \ddot{\psi}+G J_{S C} \psi^{\prime \prime}
\end{aligned}
$$

Arranging the force and moment equations into matrix form produces the following.

$$
\left\{\begin{array}{c}
-\frac{d}{d x} F_{\infty}(t) \\
-\frac{d}{d x} M_{\infty}(t)
\end{array}\right\}=\left[\begin{array}{ll}
\Upsilon_{1,1} & \Upsilon_{1,2} \\
\Upsilon_{2,1} & \Upsilon_{2,2}
\end{array}\right]\left\{\begin{array}{c}
v \\
\psi
\end{array}\right\}
$$

The matrix entries, $\Upsilon_{1,1}$, etc., are shown in Equations 4.21 through 4.24 .

$$
\begin{gathered}
\Upsilon_{1,1}=\rho A_{b}\left(\dot{\alpha}^{2} \cos ^{2} \theta-\frac{\ddot{v}}{v}-\dot{\alpha}^{2}\left[\frac{v^{\prime \prime}}{v}\left[R(L-x)+\frac{1}{2}\left(L^{2}-x^{2}\right)\right]-\frac{v^{\prime}}{v}(R+x)\right]\right)-E_{b} I_{S C} \frac{v^{\prime \prime \prime \prime}}{v} \\
\Upsilon_{1,2}=S_{z} \rho A_{b}\left(\dot{\alpha}^{2} \cos ^{2} \theta-\frac{\ddot{\psi}}{\psi}\right) \\
\Upsilon_{2,1}=S_{z} \rho A_{b}\left(\dot{\alpha}^{2} \cos ^{2} \theta-\frac{\ddot{v}}{v}\right) \\
\Upsilon_{2,2}=S_{z} \rho A_{b} \dot{\alpha}^{2} \cos ^{2} \theta-\rho J_{S C} \frac{\ddot{\psi}}{\psi}+G J_{S C} \frac{\psi^{\prime \prime}}{\psi}
\end{gathered}
$$

Note that in Equation 4.20 the off-diagonal terms in the matrix only exist if the shear centre offset is non-zero. Note also that as long as one is assuming the same 
variation in time (synchronous motion) for both the $v$ and $\psi$ dependent variables, then $\frac{\ddot{v}}{v}=\frac{\ddot{\psi}}{\psi}$, and the dynamic matrix is symmetric as required for the conservative system (damping is neglected).

Also from Hamilton's Principle, the boundary conditions of the system were found as in Equation 4.25 .

$$
\begin{array}{r}
\left.E_{b} I_{S C} v^{\prime \prime \prime}(x, t) \delta v\right|_{0} ^{L}+\rho A_{b} \dot{\alpha}^{2}\left[\left.\left[R(L-x)+\frac{1}{2}\left(L^{2}-x^{2}\right)\right] v^{\prime}(x, t) \delta v\right|_{0} ^{L}=0\right. \\
\left.E_{b} I_{S C} v^{\prime \prime}(x, t) \delta v^{\prime}\right|_{0} ^{L}=0 \\
\left.G J_{S C} \psi^{\prime}(x, t) \delta \psi\right|_{0} ^{L}=0
\end{array}
$$

These boundary condition equations apply for all of the multitude of beam end conditions; fixed-fixed, fixed-pin, pin-pin, etc. The rotating beam situation is a fixedfree condition. Using the prescribed end conditions, the boundary conditions in Equation 4.25 were simplified. The displacement at the beam root must be zero as it is cantilevered to a rigid disk. This indicated that the variation of any deflection at the root must be zero; $\delta v(x=0, t)=0$ and $\delta \psi(x=0, t)=0$. Due to the cantilever assumption, the slope of the deflection at the blade root must also be zero, $\delta v^{\prime}(x=0, t)=0$. These conditions, which apply for a rotating cantilever beam attached to a rigid hub, simplified the boundary conditions at $x=0$ to the following.

$$
\begin{aligned}
& \delta v(x=0, t)=0 \\
& \delta v^{\prime}(x=0, t)=0 \\
& \delta \psi(x=0, t)=0
\end{aligned}
$$

The free end condition that occurs at the blade tip, $x=L$, provides common conditions that must be met. The first states that there can be no shear force at a free end, $v^{\prime \prime \prime}(x=L, t)=0$. The second remarks that the bending moment at the free 
end must be zero, $v^{\prime \prime}(x=L, t)=0$. The third provides that the rate change of the twist angle, due to torque, at the free end must be zero, $\psi^{\prime}(x=L, t)=0$. These conditions are shown in Equation 4.27.

$$
\begin{aligned}
& v^{\prime \prime \prime}(x=L, t)=0 \\
& v^{\prime \prime}(x=L, t)=0 \\
& \psi^{\prime}(x=L, t)=0
\end{aligned}
$$

Note that with the end condition constraints shown in Equations 4.26 and 4.27, the Equations in 4.25 are all satisfied.

At this stage the equations of motions and the boundary conditions have been specified. At this point, the solution of the problem can proceed by employing an approximate method. If either the Rayleigh-Ritz or Galerkin methods were adopted, the two dependent variables, $v$ and $\psi$, would be expanded into a series of admissible functions that would satisfy the blade boundary conditions. An eigenvalue (freevibration) problem could then be formulated for solving the natural frequencies and corresponding modal displacements of the system, setting the aerodynamic loads equal to zero on the right-hand side of Equation 4.20 .

Due to the modal orthogonality property [17], the result of such analysis will produce a set of decoupled ordinary differential equations in the time independent variable, where the modal amplitudes, $(\{\eta(t)\})$, are the only remaining dependent variables of the problem.

$$
[M]\{\ddot{\eta}(t)\}+[K]\{\eta(t)\}=\{Q(t)\}
$$

Note that in Equations $4.28[M]$ is the diagonal matrix of generalized masses, $[K]$ is the diagonal matrix of generalized stiffnesses, and $\{Q(t)\}$ is the vector of generalized aerodynamic forces and moments acting on the natural modes of the system. These 
equations can be normalized by imposing that the matrix of generalized masses is the unit matrix, $[I]$. In this case, the system of equations can be further simplified:

$$
\{\ddot{\eta}(t)\}+\left[\omega_{n}^{2}\right]\{\eta(t)\}=\{\Psi(t)\}
$$

where $\left\{\omega_{n}^{2}\right\}$ is the diagonal matrix of the squares of the natural frequencies in $\mathrm{rad} / \mathrm{s}$ and $\{\Psi(t)\}$ is the normalized vector of generalized aerodynamic forces and moments acting on the natural modes. Therefore, the sought transfer functions of the aeroelastic system, relating the unsteady aerodynamic forces to the modal amplitudes - from which the forced response and the stability of the system can be studied, will be determined by the Laplace transformation of Equation 4.29, shown in Equation 4.30 .

$$
\{\eta(s)\}=\left(s^{2}[I]+\left[\omega^{2}\right]\right)^{-1}\{\Psi(s)\}
$$

However, the described procedure does not need to be pursued in the present work, as Equation 4.30 can also be obtained using the Finite Element Method. In the present work, the free-vibration eigenvalue problem will be solved using ANSYS, which is expected to produce an equivalent and more accurate solution for the structural problem formulated in the present chapter.

Therefore, in the next chapter, the transfer functions of the aeroelastic system will be determined directly using the solution given by ANSYS for the structural eigenvalue problem. Similarly, the unsteady aerodynamics transfer functions will be generated by a reduced-order model based on the fitting of loads produced by another commercial software, CFX. 


\section{Chapter 5}

\section{AEROELASTIC TRANSFER FUNCTION}

\subsection{Modal Analysis - Constructing the Eigenfunc- tion}

In the preceding chapters the structure and aerodynamic properties of the rotating beam system were defined. This chapter serves to combine this data into a transfer function that describes the aeroelastic system. One begins with the modified undamped field equation, Equation 5.1.

$$
\left([u]^{T}[M][u]\right)\{\ddot{\eta}(t)\}+\left([u]^{T}[K][u]\right)\{\eta(t)\}=[u]^{T}\{Q(t)\}
$$

The aerodynamics, $\{Q(t)\}$, is broken up in to the transient $([A(t)]\{\eta(t)\})$ and steady-state $\left(\left\{Q_{s s}\right\}\right)$ portions.

$$
\left([u]^{T}[M][u]\right)\{\ddot{\eta}(t)\}+\left([u]^{T}[K][u]\right)\{\eta(t)\}=[u]^{T}\left([A(t)]\{\eta(t)\}+\left\{Q_{s s}\right\}\right)
$$

As ANSYS normalizes the nodal deflection matrix, $[u]$, to the mass matrix, the following is true. 


$$
[u]^{T}[M][u]=[I]
$$

If Equation 5.3 is true, it can be seen that the $[u]^{T}[K][u]$ matrix produces the matrix of the square of the eigenvalues. Therefore, in considering $n$ mode shapes, Equation 5.1 becomes Equation 5.4.

$$
[I]\{\ddot{\eta}(t)\}+\left[\begin{array}{cccc}
\omega_{1}^{2} & 0 & \ldots & \\
& & & \\
0 & \ddots & & \\
\vdots & & & \omega_{n}^{2}
\end{array}\right]\{\eta(t)\}=[u]^{T}\left([A(t)]\{\eta(t)\}+\left\{Q_{s s}\right\}\right)
$$

The variable $n$ indicates the number of modes considered, while the variable $N$ indicates the number of nodes considered. Equation 5.5 shows the general form of the $[u]$ matrix.

$$
[u]_{2 N \times n}=\left[\begin{array}{ccc}
\left\{\Delta y_{0_{\text {mode } 1}}\right\} & \ldots & \left\{\Delta y_{0_{\text {mode } n}}\right\} \\
\left\{\Delta z_{0_{\text {mode } 1}}\right\} & \ldots & \left\{\Delta z_{0_{\text {mode } n}}\right\}
\end{array}\right]
$$

The order of the $[\mathrm{u}]$ vector is $2 N \times n$, when the system has 2 degrees of freedom, in this work these are $y_{0}$, and $z_{0}$.

The $\left\{Q_{s s}\right\}$ vector contains the steady-state aerodynamic forces for each degree of freedom at each node. This information was obtainable from the steady-state CFX simulation results.

The last portion of the equation that was defined was the $[A(t)]$ matrix. This matrix is composed of the aerodynamic loads applied at each node. Each node experiences an aerodynamic force associated with each of the structural modes considered. Therefore, as this work deals with three natural modes, the $[A(t)]$ matrix will have 
three columns. This matrix was also constructed using the aerodynamic loads determined from CFX. The general form of this equation is shown in Equation 5.6, for 3 mode shapes and 2 degrees of freedom at each node.

$$
[A(t)]=\left[\begin{array}{ccc}
a_{1,1}(t) & a_{1,2}(t) & a_{1,3}(t) \\
a_{2,1}(t) & a_{2,2}(t) & a_{2,3}(t) \\
a_{3,1}(t) & a_{3,2}(t) & a_{3,3}(t) \\
\vdots & \vdots & \vdots \\
a_{2 N, 1}(t) & a_{2 N, 2}(t) & a_{2 N, 3}(t)
\end{array}\right]
$$

The elements of the $[A(t)]$ matrix were constructed from the transient portion of the CFX output. It was assumed that the aerodynamic force is distributed uniformly along the blade span. Therefore, at each node the force applied is the total force divided by the number of nodes considered $(N)$. Each column in the $[A(t)]$ matrix represents one of the considered modes. The first $N$ rows represent the force in the direction of the first degree of freedom at each node. These rows are identical due to the uniform load distribution assumption. Similar comments can be made about the last $N$ rows, which represent the force in the direction of the second degree of freedom at each node. Equations 5.7 and 5.8 describe the entries of the $[A(t)]$ matrix.

$$
\begin{array}{r}
a_{1,1}(t)=a_{2,1}(t)=\ldots=a_{N, 1}(t)=\frac{M 1 F_{y_{0 t r}}(t)}{N} \\
a_{N+1,1}(t)=a_{N+2,1}(t)=\ldots=a_{2 N, 1}(t)=\frac{M 1 F_{z_{0_{t r}}}(t)}{N} \\
a_{1,2}(t)=a_{2,2}(t)=\ldots=a_{N, 2}(t)=\frac{M 2 F_{y 0_{t r}}(t)}{N}
\end{array}
$$




$$
\begin{array}{r}
a_{N+1,2}(t)=a_{N+2,2}(t)=\ldots=a_{2 N, 2}=\frac{M 2 F_{z_{0 t r}}(t)}{N} \\
a_{1,3}(t)=a_{2,3}(t)=\ldots=a_{N, 3}(t)=\frac{M 3 F_{y_{0 t r}}(t)}{N} \\
a_{N+1,3}(t)=a_{N+2,3}(t)=\ldots=a_{2 N, 3}(t)=\frac{M 3 F_{z_{0 t r}}(t)}{N}
\end{array}
$$

The $[u]^{T}[A(t)]$ matrix can be written as shown in Equations 5.9, 5.10, and 5.11, which has been broken up by column for easier viewing.

$$
\begin{aligned}
& \left([u]^{T}[A(t)]\right)_{1}=\left\{\begin{array}{l}
\sum_{j=1}^{N} u_{1, j}^{T} \frac{M 1 F_{y_{t r}}(t)}{N}+\sum_{j=N+1}^{2 N} u_{1, j}^{T} \frac{M 1 F_{z_{0_{r}}}(t)}{N} \\
\sum_{j=1}^{N} u_{2, j}^{T} \frac{M 1 F_{y_{0_{r r}}}(t)}{N}+\sum_{j=N+1}^{2 N} u_{2, j}^{T} \frac{M 1 F_{z_{0_{t r}}}(t)}{N} \\
\sum_{j=1}^{N} u_{3, j}^{T} \frac{M 1 F_{y_{t r}}(t)}{N}+\sum_{j=N+1}^{2 N} u_{3, j}^{T} \frac{M 1 F_{z_{0_{t r}}}(t)}{N}
\end{array}\right\} \\
& \left([u]^{T}[A(t)]\right)_{2}=\left\{\begin{array}{l}
\sum_{j=1}^{N} u_{1, j}^{T} \frac{M 2 F_{y_{t r}}(t)}{N}+\sum_{j=N+1}^{2 N} u_{1, j}^{T} \frac{M 2 F_{z_{0 t r}}(t)}{N} \\
\sum_{j=1}^{N} u_{2, j}^{T} \frac{M 2 F_{y 0_{t r}}(t)}{N}+\sum_{j=N+1}^{2 N} u_{2, j}^{T} \frac{M 2 F_{z_{t r}}(t)}{N} \\
\sum_{j=1}^{N} u_{3, j}^{T} \frac{M 2 F_{y_{0 t r}}(t)}{N}+\sum_{j=N+1}^{2 N} u_{3, j}^{T} \frac{M 2 F_{z_{0 t r}}(t)}{N}
\end{array}\right\} \\
& \left([u]^{T}[A(t)]\right)_{3}=\left\{\begin{array}{l}
\sum_{j=1}^{N} u_{1, j}^{T} \frac{M 3 F_{y_{0 t r}}(t)}{N}+\sum_{j=N+1}^{2 N} u_{1, j}^{T} \frac{M 3 F_{z_{t r}}(t)}{N} \\
\sum_{j=1}^{N} u_{2, j}^{T} \frac{M 3 F_{y_{t r}}(t)}{N}+\sum_{j=N+1}^{2 N} u_{2, j}^{T} \frac{M 3 F_{z_{t r}}(t)}{N} \\
\sum_{j=1}^{N} u_{3, j}^{T} \frac{M 3 F_{y_{0 t r}}(t)}{N}+\sum_{j=N+1}^{2 N} u_{3, j}^{T} \frac{M 3 F_{z_{t r}}(t)}{N}
\end{array}\right\}
\end{aligned}
$$

The transient portion of the forces are described in Equations 5.12. Note that each force in Equations 5.12 and 5.13 has been multiplied by a constant, 248. This 
constant represents the blade length divided by the domain thickness used in the CFX simulations. This scaling factor transforms the 2-D CFX results into full blade aerodynamic loads. The steady-state portion of the loads has been removed from the equations: $248(0.266)$ newtons for forces in the $y_{0}$ direction, and $248(-0.295)$ newtons for forces in the $z_{0}$ direction. Note also that each load is divided by a step function. In the case of the $y_{0}$ force for the first mode the function is $-0.160952 U(t)$. This number corresponds to the CFX step displacement input in the $y_{0}$ direction used to create the transient pressures in the fluid due to mode 1.

$$
\begin{aligned}
M 1 F_{y_{0_{t r}}}(t)= & \frac{248}{-0.160952 U(t)}\left(0.035 e^{-1,100 t} \cos (12,500 \pi t+0.004(2 \pi))-0.00175\right) \\
M 2 F_{y_{0_{t r}}}(t)= & \frac{248}{0.023645 U(t)}\left(0.050 e^{-1,000 t} \cos (12,500 \pi t)-0.045 \arctan (1,000 t)+0.028\right) \\
M 3 F_{y_{0_{t r}}}(t)= & \frac{248}{0.098358 U(t)}\left(0.060 e^{-1,200 t} \cos (12,500 \pi t+0.550(2 \pi))\right. \\
& +0.03 \arctan (1,000 t)-0.014)
\end{aligned}
$$

$$
\begin{aligned}
M 1 F_{z_{0_{t r}}}(t)= & \frac{248}{0.158617 U(t)}\left(0.025 e^{-1,200 t} \cos (12,500 \pi t+0.55(2 \pi))\right. \\
& +0.0055 \arctan (1,000 t)-0.0036) \\
M 2 F_{z_{0_{t r}}}(t)= & \frac{248}{0.024765 U(t)}\left(0.030 e^{-1,050 t} \cos (12,500 \pi t+0.50(2 \pi))\right. \\
& +0.045 \arctan (500 t)+0.0025) \\
M 3 F_{z_{0_{t r}}}(t)= & \frac{248}{0.073808 V(t)}\left(0.030 e^{-1,050 t} \cos (12,500 \pi t+0.05(2 \pi))\right. \\
& -0.036 \arctan (750 t)+0.00075)
\end{aligned}
$$

There has been no mention thus far of the aerodynamic moment in this chapter. In this analysis the nodes used to create $[u]$ have been chosen such that they lie at 
Table 5.1: Curve fitting residual comparison

\begin{tabular}{|c|c|c|c|}
\hline \multirow{2}{*}{ Equation } & \multicolumn{2}{|c|}{ Squared Sum of the Residuals } & \multirow{2}{*}{ Percentage Change } \\
\cline { 2 - 3 } & arctan curve fit & exponential response curve fit & \\
\hline M2Fy0 & 0.855 & 0.824 & $-3.63 \%$ \\
M3Fy0 & 0.317 & 0.307 & $-3.15 \%$ \\
M1Fz0 & 0.119 & 0.119 & $0.00 \%$ \\
M2Fz0 & 0.610 & 0.623 & $+2.13 \%$ \\
M3Fz0 & 0.442 & 0.445 & $+0.67 \%$ \\
\hline
\end{tabular}

the centre of mass, which has been assumed to be coincident with the shear centre. As such, the loading created by the aerodynamic moment can be neglected.

The Laplace transform will be used to solve the modified undamped aeroelastic equations. As such, the Laplace transformation of Equations 5.12 and 5.13 must be determined. Unfortunately, the arctan function produces difficult functions in the Laplace domain. Therefore, the following substitutions have been made to facilitate the numerical solutions.

$$
\begin{aligned}
& \arctan (500 t) \rightarrow 1.315\left(1-e^{\frac{-1.5 t}{0.003284}}\right) \\
& \arctan (750 t) \rightarrow 1.375\left(1-e^{\frac{-2 t}{0.003284}}\right) \\
& \arctan (1000 t) \rightarrow 1.340\left(1-e^{\frac{-3 t}{0.003284}}\right)
\end{aligned}
$$

These modifications to the curve fit equations assume an exponential response curve instead of the fitted arctan shape. The accuracy of the curve fits were affected as shown in Table 5.1.

Indeed, Table 5.1 shows that the substitution from the arctan function to a exponential response function produced better results for two of the curve fits (those with negative percentage change values).

The modified new force functions are written in Equations 5.15 and 5.16. 


$$
\begin{aligned}
M 1 F_{y_{0_{t r}}}(t)= & \frac{248}{-0.160952 U(t)}\left(0.035 e^{-1,100 t} \cos (12,500 \pi t+0.004(2 \pi))-0.00175\right) \\
M 2 F_{y_{0_{t r}}}(t)= & \frac{248}{0.023645 U(t)}\left(0.050 e^{-1,000 t} \cos (12,500 \pi t)-0.060\left(1-e^{\frac{-3 t}{0.003284}}\right)+0.028\right) \\
M 3 F_{y_{0_{t r}}}(t)= & \frac{248}{0.098358 U(t)}\left(0.060 e^{-1,200 t} \cos (12,500 \pi t+0.550(2 \pi))\right. \\
& \left.+0.040\left(1-e^{\frac{-3 t}{0.003284}}\right)-0.014\right)
\end{aligned}
$$

$$
\begin{aligned}
M 1 F_{z_{0_{t r}}}(t)= & \frac{248}{0.158617 U(t)}\left(0.025 e^{-1,200 t} \cos (12,500 \pi t+0.55(2 \pi))\right. \\
& \left.+0.007\left(1-e^{\frac{-3 t}{0.003284}}\right)-0.0036\right) \\
M 2 F_{z_{0 t r}}(t)= & \frac{248}{0.024765 U(t)}\left(0.030 e^{-1,050 t} \cos (12,500 \pi t+0.50(2 \pi))\right. \\
& \left.+0.059\left(1-e^{\frac{-1.5 t}{0.003284}}\right)+0.0025\right) \\
M 3 F_{z_{0 t r}}(t)= & \frac{248}{0.073808 U(t)}\left(0.030 e^{-1,050 t} \cos (12,500 \pi t+0.05(2 \pi))\right. \\
& \left.-0.050\left(1-e^{\frac{-2 t}{0.003284}}\right)+0.00075\right)
\end{aligned}
$$

At this stage it is beneficial to rewrite the system equation in the Laplace domain.

$$
\begin{gathered}
{[I]\{\eta(s)\} s^{2}+\left[\begin{array}{cccc}
\omega_{1}^{2} & 0 & \ldots & \\
0 & \ddots & & \\
& & & \\
\vdots & & & \omega_{n}^{2}
\end{array}\right]\{\eta(s)\}=k[u]^{T}\left([A(s)]\{\eta(s)\}+\left[Q_{s s}\right] s^{-1}\right)} \\
\left([I] s^{2}+\left[\begin{array}{cccc}
\omega_{1}^{2} & 0 & \ldots & \\
0 & \ddots & & \\
\vdots & & & \omega_{n}^{2}
\end{array}\right]-k[u]^{T}[A(s)]\right)\{\eta(s)\}=k[u]^{T}\left[Q_{s s}\right] s^{-1}
\end{gathered}
$$


In the Laplaced form of the system equation the transient loading is represented by the $[A(s)]$ matrix, which is a function of the Laplace transform of the force equations. Note that in the above equation a gain value, $k$, has been introduced, scaling the aerodynamic terms. This scaling factor will be used to vary the amplitude, and therefore, the impact of the aerodynamic forces on the aeroelastic problem solution and therefore to assess the flutter stability of the system. When $k=0$, no aerodynamic forces are being considered. These equations are shown below.

$$
\begin{aligned}
& M 1 F_{y_{0_{t r}}}(s)=\frac{\left(-51.2156 s^{2}-150.8794 s+4.1615 \times 10^{9}\right)}{\left((s+1100)^{2}+1.5421 \times 10^{9}\right)} \\
& M 2 F_{y_{0_{t r}}}(s)=\frac{\left(524.4238 s^{3}+4.2861 \times 10^{5} s^{2}-6.7070 \times 10^{8} s-8.8712 \times 10^{14}\right)}{\left((s+1000)^{2}+1.5421 \times 10^{9}\right)(s+913.5201)} \\
& M 3 F_{y_{0_{t r}}}(s)=\frac{\left(-143.8799 s^{3}+1.6239 \times 10^{6} s^{2}+1.7405 \times 10^{9} s+1.4221 \times 10^{14}\right)}{\left((s+1200)^{2}+1.5421 \times 10^{9}\right)(s+913.5201)} \\
& M 1 F_{z_{0_{t r}}}(s)=\frac{\left(-37.1748 s^{3}+4.0576 \times 10^{5} s^{2}+4.1655 \times 10^{8} s+1.5433 \times 10^{13}\right)}{\left((s+1200)^{2}+1.5421 \times 10^{9}\right)(s+913.5201)} \\
& M 2 F_{z_{0_{t r}}}(s)=\frac{\left(-300.4240 s^{3}-1.8283 \times 10^{5} s^{2}+4.2263 \times 10^{8} s+4.1647 \times 10^{14}\right)}{\left((s+1050)^{2}+1.5421 \times 10^{9}\right)(s+456.7600)} \\
& M 3 F_{z_{0_{t r}}}(s)=\frac{\left(95.8685 s^{3}-1.1665 \times 10^{6} s^{2}-8.9853 \times 10^{8} s-1.5790 \times 10^{14}\right)}{\left((s+1050)^{2}+1.5421 \times 10^{9}\right)(s+609.0134)}
\end{aligned}
$$

The $[u]^{T}[A(s)]$ matrix was broken up by column, and shown in Equations 5.20, 5.21 and 5.22 .

$$
\left([u]^{T}[A(s)]\right)_{1}=\left\{\begin{array}{l}
\sum_{j=1}^{N} u_{1, j}^{T} \frac{M 1 F_{y_{0_{t r}}}(s)}{N}+\sum_{j=N+1}^{2 N} u_{1, j}^{T} \frac{M 1 F_{z_{t r}}(s)}{N} \\
\sum_{j=1}^{N} u_{2, j}^{T} \frac{M 1 F_{y_{0_{t r}}}(s)}{N}+\sum_{j=N+1}^{2 N} u_{2, j}^{T} \frac{M 1 F_{z 0_{t r}}(s)}{N} \\
\sum_{j=1}^{N} u_{3, j}^{T} \frac{M 1 F_{y_{0_{t r}}}(s)}{N}+\sum_{j=N+1}^{2 N} u_{3, j}^{T} \frac{M 1 F_{z 0_{t r}}(s)}{N}
\end{array}\right\}
$$




$$
\begin{aligned}
&\left([u]^{T}[A(s)]\right)_{2}=\left\{\begin{array}{l}
\sum_{j=1}^{N} u_{1, j}^{T} \frac{M 2 F_{y_{0_{t r}}}(s)}{N}+\sum_{j=N+1}^{2 N} u_{1, j}^{T} \frac{M 2 F_{z_{0_{t r}}}(s)}{N} \\
\sum_{j=1}^{N} u_{2, j}^{T} \frac{M 2 F_{y_{0_{t r}}}(s)}{N}+\sum_{j=N+1}^{2 N} u_{2, j}^{T} \frac{M 2 F_{z_{0_{t r}}}(s)}{N} \\
\sum_{j=1}^{N} u_{3, j}^{T} \frac{M 2 F_{y_{0_{t r}}}(s)}{N}+\sum_{j=N+1}^{2 N} u_{3, j}^{T} \frac{M 2 F_{z_{0_{t r}}}(s)}{N}
\end{array}\right\} \\
&\left([u]^{T}[A(s)]\right)_{3}=\left\{\begin{array}{l}
\sum_{j=1}^{N} u_{1, j}^{T} \frac{M 3 F_{y_{0_{t r}}}(s)}{N}+\sum_{j=N+1}^{2 N} u_{1, j}^{T} \frac{M 3 F_{z_{0_{t r}}}(s)}{N} \\
\sum_{j=1}^{N} u_{2, j}^{T} \frac{M 3 F_{y_{0_{t r}}}(s)}{N}+\sum_{j=N+1}^{2 N} u_{2, j}^{T} \frac{M 3 F_{z_{0_{t r}}}(s)}{N} \\
\sum_{j=1}^{N} u_{3, j}^{T} \frac{M 3 F_{y_{0_{t r}}}(s)}{N}+\sum_{j=N+1}^{2 N} u_{3, j}^{T} \frac{M 3 F_{z_{0_{t r}}}(s)}{N}
\end{array}\right\}
\end{aligned}
$$

The following matrix can also be written.

$$
\begin{aligned}
& \left([I] s^{2}+[u]^{T}[K][u]-k[u]^{T}[A(s)]\right)_{1} \\
& =\left\{\begin{array}{c}
s^{2}+\omega_{1}^{2}-k \sum_{j=1}^{N} u_{1, j}^{T} \frac{M 1 F_{y_{0_{t r}}}(s)}{N}-k \sum_{j=N+1}^{2 N} u_{1, j}^{T} \frac{M 1 F_{z_{0_{t r}}}(s)}{N} \\
-k \sum_{j=1}^{N} u_{2, j}^{T} \frac{M 1 F_{y_{0_{t r}}}(s)}{N}-k \sum_{j=N+1}^{2 N} u_{2, j}^{T} \frac{M 1 F_{z_{0_{t r}}}(s)}{N} \\
-k \sum_{j=1}^{N} u_{3, j}^{T} \frac{M 1 F_{y_{0_{t r}}}(s)}{N}-k \sum_{j=N+1}^{2 N} u_{3, j}^{T} \frac{M 1 F_{z_{0 t r}}(s)}{N}
\end{array}\right\} \\
& \left([I] s^{2}+[u]^{T}[K][u]-k[u]^{T}[A(s)]\right)_{2} \\
& =\left\{\begin{array}{c}
-k \sum_{j=1}^{N} u_{1, j}^{T} \frac{M 2 F_{y_{0_{t r}}}(s)}{N}-k \sum_{j=N+1}^{2 N} u_{1, j}^{T} \frac{M 2 F_{z_{0_{t r}}}(s)}{N} \\
s^{2}+\omega_{2}^{2}-k \sum_{j=1}^{N} u_{2, j}^{T} \frac{M 2 F_{y_{0_{t r}}}(s)}{N}-k \sum_{j=N+1}^{2 N} u_{2, j}^{T} \frac{M 2 F_{z_{0_{t r}}}(s)}{N} \\
-k \sum_{j=1}^{N} u_{3, j}^{T} \frac{M 2 F_{y_{0_{t r}}}(s)}{N}-k \sum_{j=N+1}^{2 N} u_{3, j}^{T} \frac{M 2 F_{z_{0 t r}}}{N}(s)
\end{array}\right\}
\end{aligned}
$$




$$
\left.\begin{array}{l}
\left([I] s^{2}+[u]^{T}[K][u]-k[u]^{T}[A(s)]\right)_{3} \\
-k \sum_{j=1}^{N} u_{1, j}^{T} \frac{M 3 F_{y_{0_{t r}}}(s)}{N}-k \sum_{j=N+1}^{2 N} u_{1, j}^{T} \frac{M 3 F_{z_{0_{t r}}}(s)}{N} \\
-k \sum_{j=1}^{N} u_{2, j}^{T} \frac{M 3 F_{y_{0 t r}}(s)}{N}-k \sum_{j=N+1}^{2 N} u_{2, j}^{T} \frac{M 3 F_{z_{0_{t r}}}(s)}{N} \\
s^{2}+\omega_{3}^{2}-k \sum_{j=1}^{N} u_{3, j} \frac{M 3 F_{y_{0 t r}}(s)}{N}-k \sum_{j=N+1}^{2 N} u_{3, j}^{T} \frac{M 3 F_{z_{0 t r}}(s)}{N}
\end{array}\right\}
$$

For the situation being examined, 13 nodes $(N=13)$ have been chosen along the blade span at the centre of mass. Equations 5.26, 5.27, and 5.28 result from using $N=13$ with the modal matrix, $[u]$, described by ANSYS and shown in Appendix E.

$$
\begin{aligned}
& \left([I] s^{2}+[u]^{T}[K][u]-k[u]^{T}[A(s)]\right)_{1} \\
& =\left\{\begin{array}{c}
s^{2}+\omega_{1}^{2}-k(-90.0769) M 1 F_{y_{0_{t r}}}(s)-k(88.4154) M 1 F_{z_{0_{t r}}}(s) \\
-k(41.4524) M 1 F_{y_{0_{t r}}}(s)-k(31.8167) M 1 F_{z_{0_{t r}}}(s) \\
-k(80.0154) M 1 F_{y_{0_{t r}}}(s)-k(80.2385) M 1 F_{z_{0 t r}}(s)
\end{array}\right\} \\
& \left([I] s^{2}+[u]^{T}[K][u]-k[u]^{T}[A(s)]\right)_{2} \\
& =\left\{\begin{array}{c}
-k(-90.0769) M 2 F_{y_{0_{t r}}}(s)-k(88.4154) M 2 F_{z_{0_{t r}}}(s) \\
s^{2}+\omega_{2}^{2}-k(41.4524) M 2 F_{y_{0_{t r}}}(s)-k(31.8167) M 2 F_{z_{0_{t r}}}(s) \\
-k(80.0154) M 2 F_{y_{0_{t r}}}(s)-k(80.2385) M 2 F_{z_{0_{t r}}}(s)
\end{array}\right\}
\end{aligned}
$$




$$
\left.\begin{array}{l}
\left([I] s^{2}+[u]^{T}[K][u]-k[u]^{T}[A(s)]\right)_{3} \\
-k(-90.0769) M 3 F_{y_{0_{t r}}}(s)-k(88.4154) M 3 F_{z_{0_{t r}}}(s) \\
-k(41.4524) M 3 F_{y_{0_{t r}}}(s)-k(31.8167) M 3 F_{z_{0_{t r}}}(s) \\
s^{2}+\omega_{3}^{2}-k(80.0154) M 3 F_{y_{0_{t r}}}(s)-k(80.2385) M 3 F_{z_{0_{t r}}}(s)
\end{array}\right\}
$$

At this stage the determinant of the $\left([I] s^{2}+[u]^{T}[K][u]-k[u]^{T}[A(s)]\right)$ matrix was determined. It corresponds to the denominator of the aeroelastic transfer function, $T F_{\text {den }}$. 


$$
\begin{aligned}
T F_{d e n}= & {\left[\left\{s^{2}+\omega_{1}^{2}-k(-90.0769) M 1 F_{y_{0_{t r}}}(s)-k(88.4154) M 1 F_{z_{0_{t r}}}(s)\right\}\right.} \\
& \left\{\left(s^{2}+\omega_{2}^{2}-k(41.4524) M 2 F_{y_{0_{t r}}}(s)-k(31.8167) M 2 F_{z_{0_{t r}}}(s)\right)\right. \\
& \left(s^{2}+\omega_{3}^{2}-k(80.0154) M 3 F_{y_{0_{t r}}}(s)-k(80.2385) M 3 F_{z_{0_{t r}}}(s)\right) \\
& -\left(-k(41.4524) M 3 F_{y_{0_{t r}}}(s)-k(31.8167) M 3 F_{z_{0_{t r}}}(s)\right) \\
& \left.\left.\left(-k(80.0154) M 2 F_{y_{0_{t r}}}(s)-k(80.2385) M 2 F_{z_{0_{t r}}}(s)\right)\right\}\right] \\
& -\left[\left\{-k(-90.0769) M 2 F_{y_{0_{t r}}}(s)-k(88.4154) M 2 F_{z_{0_{t r}}}(s)\right\}\right. \\
& \left\{\left(-k(41.4524) M 1 F_{y_{0_{t r}}}(s)-k(31.8167) M 1 F_{z_{0_{t r}}}(s)\right)\right. \\
& \left(s^{2}+\omega_{3}^{2}-k(80.0154) M 3 F_{y_{0_{t r}}}(s)-k(80.2385) M 3 F_{z_{0_{t r}}}(s)\right) \\
& -\left(-k(41.4524) M 3 F_{y_{0_{t r}}}(s)-k(31.8167) M 3 F_{z_{0_{t r}}}(s)\right) \\
& \left.\left.\left(-k(80.0154) M 1 F_{y_{0_{t r}}}(s)-k(80.2385) M 1 F_{z_{0_{t r}}}(s)\right)\right\}\right] \\
& +\left[\left\{-k(-90.0769) M 3 F_{y_{0_{t r}}}(s)-k(88.4154) M 3 F_{z_{0_{t r}}}(s)\right\}\right. \\
& \left.\left.\left(-k(80.0154) M 1 F_{y_{0_{t r}}}(s)-k(80.2385) M 1 F_{z_{0_{t r}}}(s)\right)\right\}\right] \\
& \left(-k(41.4524) M 1 F_{y_{0_{t r}}}(s)-k(31.8167) M 1 F_{z_{0_{t r}}}(s)\right) \\
& \left(-k(80.0154) M 2 F_{y_{0_{t r}}}(s)-k(80.2385) M 2 F_{z_{0_{t r}}}(s)\right) \\
& \left(s^{2}+\omega_{2}^{2}-k(41.4524) M 2 F_{y_{0_{t r}}}(s)-k(31.8167) M 2 F_{z_{0_{t r}}}(s)\right) \\
& \\
& \\
& \\
& \\
&
\end{aligned}
$$

Substituting the equations for the aerodynamic forces, and rearranging the results produces the denominator of the aeroelastic transfer function for the system. The following equation, Equation 5.30, shows this denominator at zero gain, $k=0$. With a non-zero gain value the equation is quite large, and has therefore, not been displayed.

$$
T F_{d e n_{k=0}}=\left(s^{2}+\omega_{1}^{2}\right)\left(s^{2}+\omega_{1}^{2}\right)\left(s^{2}+\omega_{1}^{2}\right)
$$


A zero gain value $(k=0)$ was used to check for the natural frequencies of the freevibration problem. In the general case, when $k$ is not zero, setting the determinant equation equal to zero produces a method of determining the instability points of the aeroelastic system. With $k=0$, the roots found are shown in Table 5.2. The frequencies found match the natural frequencies of the structure determined using ANSYS, Chapter 2.

Table 5.2: Roots of the system equation determinant, $k=0$

\begin{tabular}{|c|c|c|}
\hline \multicolumn{3}{|c|}{$k=0$} \\
\hline real part & imaginary part & frequency $[\mathrm{Hz}]$ \\
\hline 0 & $\pm 8413 i$ & 1339 \\
0 & $\pm 16814 i$ & 2676 \\
0 & $\pm 29682 i$ & 4724 \\
\hline
\end{tabular}

With the full aerodynamics, $k=1$, similar roots were calculated, shown in Table 5.3 .

Tables 5.2 and 5.3 show that the roots for the case when there are no aerodynamics produce identical results to those with full aerodynamics. If the aerodynamic loads are largely exaggerated, the roots change as shown in Table 5.4.

This analysis shows that the aerodynamics do not affect the stability of the system. Note also that the real values of the roots are all negative, indicating a stable system at all $k$-gain factors between zero and one. When the aerodynamic loads are largely exaggerated, there exists three unstable roots as shown in Table 5.4. 
Table 5.3: Roots of the system equation determinant, $k=1$

\begin{tabular}{|c|c|c|}
\hline \multicolumn{3}{|c|}{$k=1$} \\
\hline real part & imaginary part & frequency $[\mathrm{Hz}]$ \\
\hline-457 & $0 i$ & 0 \\
-914 & $0 i$ & 0 \\
-1370 & $0 i$ & 0 \\
0 & $\pm 8413 i$ & 1339 \\
0 & $\pm 16814 i$ & 2676 \\
0 & $\pm 29682 i$ & 4724 \\
-1000 & $\pm 39270 i$ & 6250 \\
-1050 & $\pm 39270 i$ & 6250 \\
-1100 & $\pm 39270 i$ & 6250 \\
-1200 & $\pm 39270 i$ & 6250 \\
\hline
\end{tabular}

Table 5.4: Roots of the system equation determinant, $k=100,000$

\begin{tabular}{|c|c|c|}
\hline \multicolumn{3}{|c|}{$k=100,000$} \\
\hline real part & imaginary part & frequency $[\mathrm{Hz}]$ \\
\hline-302 & $0 i$ & 0 \\
-786 & $0 i$ & 0 \\
-1295 & $0 i$ & 0 \\
-1576 & $0 i$ & 0 \\
18 & $\pm 8527 i$ & 1357 \\
296 & $\pm 17678 i$ & 2814 \\
212 & $\pm 28527 i$ & 4540 \\
-2279 & $\pm 38779 i$ & 6172 \\
-1088 & $\pm 39103 i$ & 6223 \\
-420 & $\pm 39256 i$ & 6248 \\
-1082 & $\pm 39281 i$ & 6252 \\
-1053 & $\pm 39471 i$ & 6282 \\
\hline
\end{tabular}




\section{Chapter 6}

\section{CONCLUSION, FUTURE WORK AND RECOMMENDATIONS}

\subsection{Summary}

A Pro/Engineer model of the test blade was created and imported into ANSYS10.0. The blade was meshed and given material properties. The blade root was restrained in all directions, and a global rotational velocity was defined. The stress state was calculated by ANSYS and used to calculate the structural mode shapes. Post-processing transformed the ANSYS mode shape output into simple midspan displacements, one set for each of the three modes (first bending, first torsion, and first lead/lag).

The unsteady aerodynamic forces were calculated using CFX. A blade passage domain was created and meshed to produce a flow volume for use in CFX. The domain modelled a thin slice of the blade passage at the midspan of the turbine blade. A steady-state flow field solution was solved using the turbine operating parameters and CFX-10.0. The steady-state solution was used as the initial state for a transient simulation, in which the blade walls were displaced to reflect each of the first three structural mode shapes determined using the ANSYS software (in three separate 
transient simulations). A grid convergence check, as well as a timestep independence check were performed to aid in ensuring reasonable results.

The CFX simulation output was checked several ways. One check involved comparing the results to an estimation of the resultant force in the $y$ direction. The solver output plots were also verified using the post-processor output and a Matlab code. Equations were fit to the verified data in order to produce functions that describe the aeroelastic forces on the blade in the $y_{0}$ and $z_{0}$ directions.

The field equations for the system were derived analytically and the aeroelastic problem was formulated in the frequency (Laplace) domain. The rotating hub-blade system consists of a cantilevered blade attached to a hub rotating at a constant angular velocity. The properties of the blade and hub material were known and were assumed to be isotropic. The cantilevered blade was treated as a uniform EulerBernoulli beam. This derivation served as an introduction to the modal analysis.

The equations fit using CFX were used in conjunction with the structural ANSYS results to produce an aeroelastic transfer function that describes the aeroelastic mechanism of the test blade at its operating conditions.

\subsection{Conclusion}

After following the methodology to provide the transfer function for the internal flow system, the transfer function denominator was tested for stability. The stability results were found using different aerodynamic gain factors. With no aerodynamic gain, the system had three poles, which corresponded to the structural modes of vibration.

The aerodynamics of the system are fully applied when the aerodynamic gain is given a value of one. As expected, there are more poles of the aeroelastic transfer function when aerodynamic forces exist. In this situation there was no difference in 
the frequency of the aeroelastic modes that correspond to the the natural structural modes of vibration $(1339 \mathrm{~Hz}, 2676 \mathrm{~Hz}, 4727 \mathrm{~Hz})$. This is an unexpected result as one would expect the aeroelastic system to have shifted values. This, however, is not of major importance as this could be a function of the test point chosen to perform the analysis. Were the analysis to have occurred at a cross-over point on the Campbell diagram rather than at the operating point, it would be much more likely that the aeroelastic poles would differ from the structural poles. This is discussed further in Section 6.3.

\subsection{Future Work and Recommendations}

This work is a first work in the area. As such, there is much room for advancement. This section describes the logical progressions of the work.

The aeroelastic transfer function in this work was calculated at the operating speed of the engine. This speed was chosen because the aerodynamic initial conditions were available at this point. Because the goal of the work was to illustrate a method, the engine speed used was of little importance. It is very likely that the operating speed will not be the speed of interest for those who use this method to obtain useful results.

Figure 6.1 illustrates the Campbell diagram for the turbine blade system. The dotted circles emphasize the cross-over points. These are the regions where the turbine rotation matches one of the structural modes of vibration. It is these regions that are most prone to instability due to the increased possibility of exciting one of the structural modes of vibration. As such, these are the regions that must be analyzed in order to determine the stability of the system as the engine is starting up.

Future work in the CFD analysis can take place in several ways. These are discussed in the following paragraphs.

As the AGARD Manual [2] has discussed, the interblade phase angle can be an 


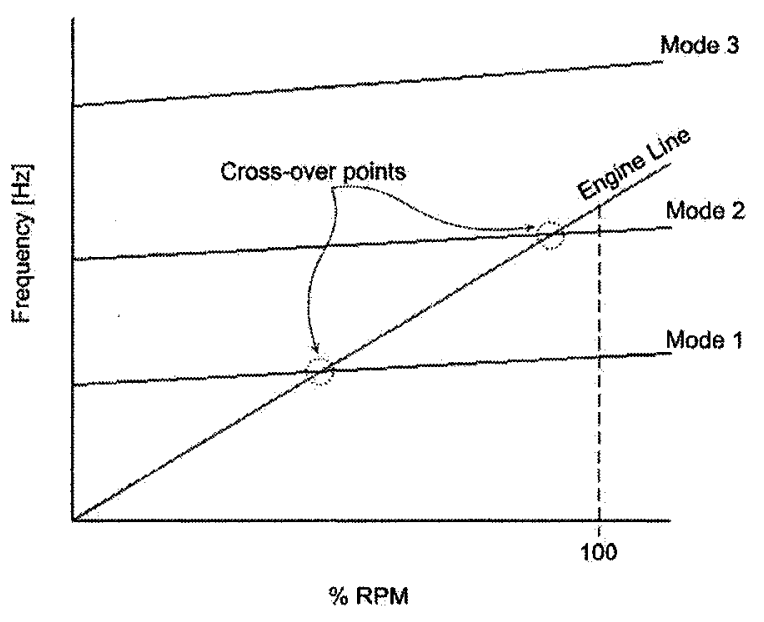

Figure 6.1: Campbell diagram

important parameter in determining stability of rotor disks. The periodic boundaries used in the CFD in this work have defined the disk to have blades that are moving in phase. In order to extend the work to incorporate interblade phase angles, the CFD could be run for multiple blades within a fluid domain. Although this is more complicated, it is feasible, and therefore the methodology can account for interblade phase angles.

The possibility of pressure waves reflecting off the opening was not investigated in this work. These reflections are not a function of the engine setup, but merely a product of the CFD solution technique. As such, these reflections are to be avoided. Non-reflective boundaries can be applied in order to remedy this solution error, and should be applied in future works when required.

The aerodynamics in a turbine are $3-\mathrm{D}$ in nature. The work presented approximates these loads in two dimensions, and then assumes a distribution along the blade span based on the 2-D loading. The methodology provided is valid for incorporating 3-D flow calculations. This situation requires more complicated CFD but will produce more accurate results. 
In the interest of time, simplifications were made in the research presented in this thesis. One of the simplifications occurred in the curve fitting process documented in Chapter 3. CFX-10.0 produced complicated transient aerodynamic data as a result of the step input perturbations associated with the natural modes of the structure. These data were approximated using oscillating decaying functions. Although the curves roughly represent the trends shown in the data, the matching could be improved. The difficulty in this process is that as a curve fit equation becomes more complicated, so too does the transfer function calculation. It is the recommendation of the author that more time be spent on representing the aerodynamic trends more effectively.

The research in this thesis has explored the possibility of applying proven external flow techniques to an internal flow system. As this is novel research, much future work is possible. 


\section{List of References}

[1] S. KENNY, "Development of a multi-disciplinary design tool for axialflow turbines," Master's thesis, Carleton University, Ottawa, Canada, 2005.

[2] M. PLATZER and F. CARTA, Aeroelasticity in Axial-Flow Turbomachinery - Volume 1: Unsteady Turbomachinery Aerodynamics. AGARD, agardograph no.298 ed., 1987.

[3] M. PLATZER and F. CARTA, Aeroelasticity in Axial-Flow Turbomachinery - Volume 2: Structural Dynamics and Aeroelasticity. AGARD, agardograph no.298 ed., 1987.

[4] L. SHROEDER and S. FLEETER, "Viscous aerodynamic analysis of an oscillating flat plate airfoil with a locally analytical solution," AIAA 26th Aerospace Science Meeting, vol. AIAA-88-0130, 1988.

[5] J. L. YANG, J.B. and D. CHEN, "Dynamic modelling and control of a rotating euler-bernoulli beam," Journal of Sound and Vibration, vol. 274, pp. 863-875, 2004 .

[6] K. HU and VLAHOPOULOS, "A finite element formulation for coupling rigid and flexible body dynamics of rotating beams," Journal or Sound and Vibration, vol. 253, no. (3), pp. 603-630, 2002.

[7] D. YAU and E. FUNG, "Dynamic response of a rotating flexible arm carrying a moving mass," Journal of Sound and Vibration, vol. 257, no. (1), pp. 107-117, 2002.

[8] E. FUNG and D. YAU, "Effects of centrifugal stiffening on the vibration frequencies of a constrained flexible arm," Journal of Sound and Vibration, vol. 244, no. (5), pp. 809-841, 1999. 
[9] H. J.-H. CAI, GUO-PING and S. X. YANG, "Model study and active control of a rotating flexible cantilever beam," International Journal of Mechanical Sciences, vol. 46, pp. 871-889, 2004.

[10] H. ZIBDEH and H. JUMA, "Dynamic response of a rotating beam subjected to a random moving load," Journal of Sound and Vibration, vol. 223, no. (5), pp. 741-758, 1999.

[11] G. JACQUET-RICHARDET and R. HENRY, "A modal aeroelastic finite element analysis method for advanced turbomachinery stages," International Journal for Numerical Methods in Engineering, vol. 37, no. 24, pp. 4205-4217, 1994.

[12] W. Silva, "Reduced-order models based on linear and nonlinear aerodynamic impulse responses," American Institute of Aeronautics and Astronautics, no. A9924653, pp. 638-348, 1999.

[13] Anonymous, ANSYS 8.1 Complete User's Manual, 2004.

[14] P. BUDGELL, ANSYS Tips [online]. Burlington, Ontario, Canada, 1998. Available from: http://www3.sympatico.ca/peter_budgell/ANSYS_tips.html [Accessed 15 May 2006].

[15] D. DARMOFAL, Structured vs. Unstructured Grids [online]. Cambridge, Massachusetts, USA, 2005. Available from: http://ocw.mit.edu [Accessed 27 January 2007]:

[16] Anonymous, CFX-5.7.1 User Manual, 2004.

[17] J. GINSBERG, Mechanical and Structural Vibrations: Theory and Applications. John Wiley \& Sons, Inc., 2001.

[18] B. YOUNG, Energy Methods of Structural Analysis: Theory, Worked Examples and Problems. The MacMillan Press Ltd., 1981.

[19] L. MEIROVITCH, Dynamics and Control of Structures. John Wiley \& Sons, Inc., 1990. 


\title{
Appendix A
}

\section{Matlab post-processing $m$-file}

\author{
function [FyOsum, FzOsum, MOOtotal, MSCtotal, dadd] =PostProcessing (inputname , outputname) \\ dadd $=0$; \\ $\%$ This file calculates the resultant forces and moment at the \\ $\%$ shear centre. These values are returned to the user as well as written to an \\ $\%$ output file. \\ $\%$ The only input is the filename of the file that you wish to postprocess and the \\ $\%$ outfile name where you wish to save the processed data. \\ data=csvread (inputname $, 5,0$ ); \\ $\%$ saves the input as 'data' in a length $\mathrm{x} 3$ matrix \\ inputlength=length (data); \\ $\%$ In order to process the data properly, replicate the first row of data is \\ $\%$ replicated and placed at the bottom of the data series. The last row of data is \\ $\%$ also replicated and placed before the first row. This is done to simplify to \\ $\%$ analysis procedure. The data series represents the Pressures around the periphery
}

80 
$\%$ of the blade. It is, therefore, a circle of data. In order to approximate the $\%$ forces associated with these pressures the coordinates before and after a given $\%$ point are used to create a direction vector upon which the pressure force acts. $\%$ Replicating the first and last entries allows a single definition for acquiring $\%$ the direction vector based solely on the values of the coordinates in the rows $\%$ above and below the given point. Therefore, we will be working with a matrix of $\%$ (inputlength+2,3) and the results will be stored as a matrix of (inputlength,6). $\%$ $\%$ $\%$ $\%$ $\%$ $\%$ These results are then summed to produce the resultant forces in the $y$, and $z$ $\%$ directions, and resultant moment about the shear centre which for mode 1 has $\%$ been calculated to be at:

$\%$

$\% \quad \operatorname{SC}(y 0, z 0)=(-0.01558,-0.01661)$

y0sc $=-0.01558 ; \quad \%$ y0 coord for shear centre

z0sc $=-0.01661 ; \quad \%$ zo coord for shear centre

datamod=zeros (inputlength+2,3);

datamod $(1,:)=\operatorname{data}($ inputlength, $:)$; datamod $(2:$ inputlength $+1,:$ ) =data;

datamod (inputlength $+2,:)=\operatorname{data}(1,:)$;

$\%$ Split data up into it's components, yo coordinate, z0 coordinate, and Static $\%$ Pressure value at this location.

yO=datamod $(:, 1) ; \mathrm{z} 0=$ datamod $(:, 2) ; \mathrm{P}=\operatorname{datamod}(:, 3)$; 
$\%$ Start a loop to calculate the contribution of nodal pressure. Remember that we $\%$ only calculate the contributions from the second row to the second last row as $\%$ the first and last rows are repeats. Initialize the nodal contribution vectors:

Fy $0=z e r o s(1$, inputlength); FzO=zeros (1, inputlength);

MySC=zeros ( 1 , inputlength); \% about the shear centre MzSC=zeros (1, inputlength); \% about the shear centre

My00=zeros ( 1 , inputlength); \% about the cartesian zero

MzO0=zeros (1,inputlength); \% about the cartesian zero

for $i=2:($ inputlength +1$)$

$\%$ Calculate the direction vector of the segment

yodirvec=yo $(i+1)-\mathrm{y} 0(i-1)$;

zodirvec $=z 0(i+1)-z 0(i-1)$;

$\%$ Translate the segment direction vector into an 'inward normal' (the pressure

$\%$ application dir)

yOpressdir=-zodirvec;

zOpressdir=yOdirvec;

$\%$ Calculate the angle the pressure direction creates with the horizontal

theta_rad=atan (abs (y0pressdir/zopressdir));

$\%$ Calculate the y0 and z0 components of pressure.

PyO $=\operatorname{abs}(P(i) * \sin ($ theta_rad));

$P z 0=\operatorname{abs}(P(i) * \cos ($ theta_rad)); 
$\%$ The distance along the blade over which this pressure will act is approximately $\%$ half of the length of the calculated direction vector.

$d=0.5 * \operatorname{sqrt}\left(\right.$ yodirvec $\left.{ }^{\wedge}+z_{0} \operatorname{dirvec}^{\wedge} 2\right)$;

dadd $=$ dadd $+\mathrm{d}$;

$\%$ We will assume that the section is the same size as the virtual domain

$\%(0.00025 m)$

$\mathrm{s}=0.00025$

$\%$ Now the forces can be calculated, remembering to check the direction in order to $\%$ determine whether the force is positive or negative.

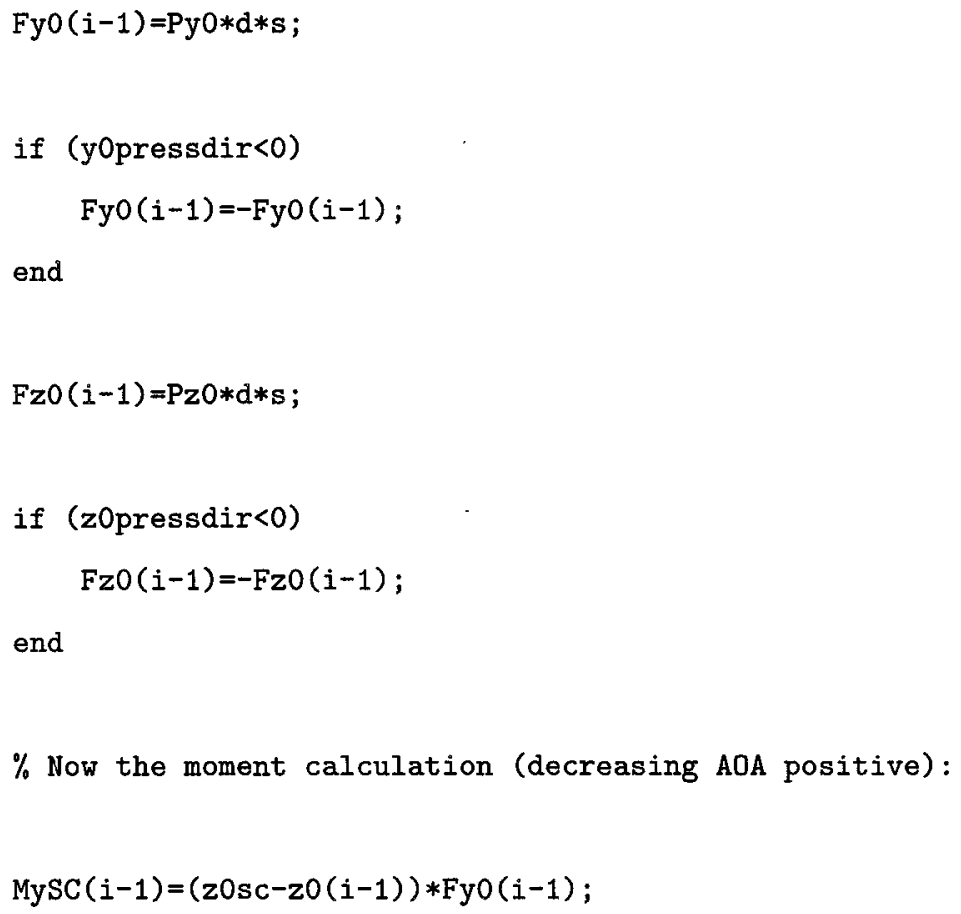




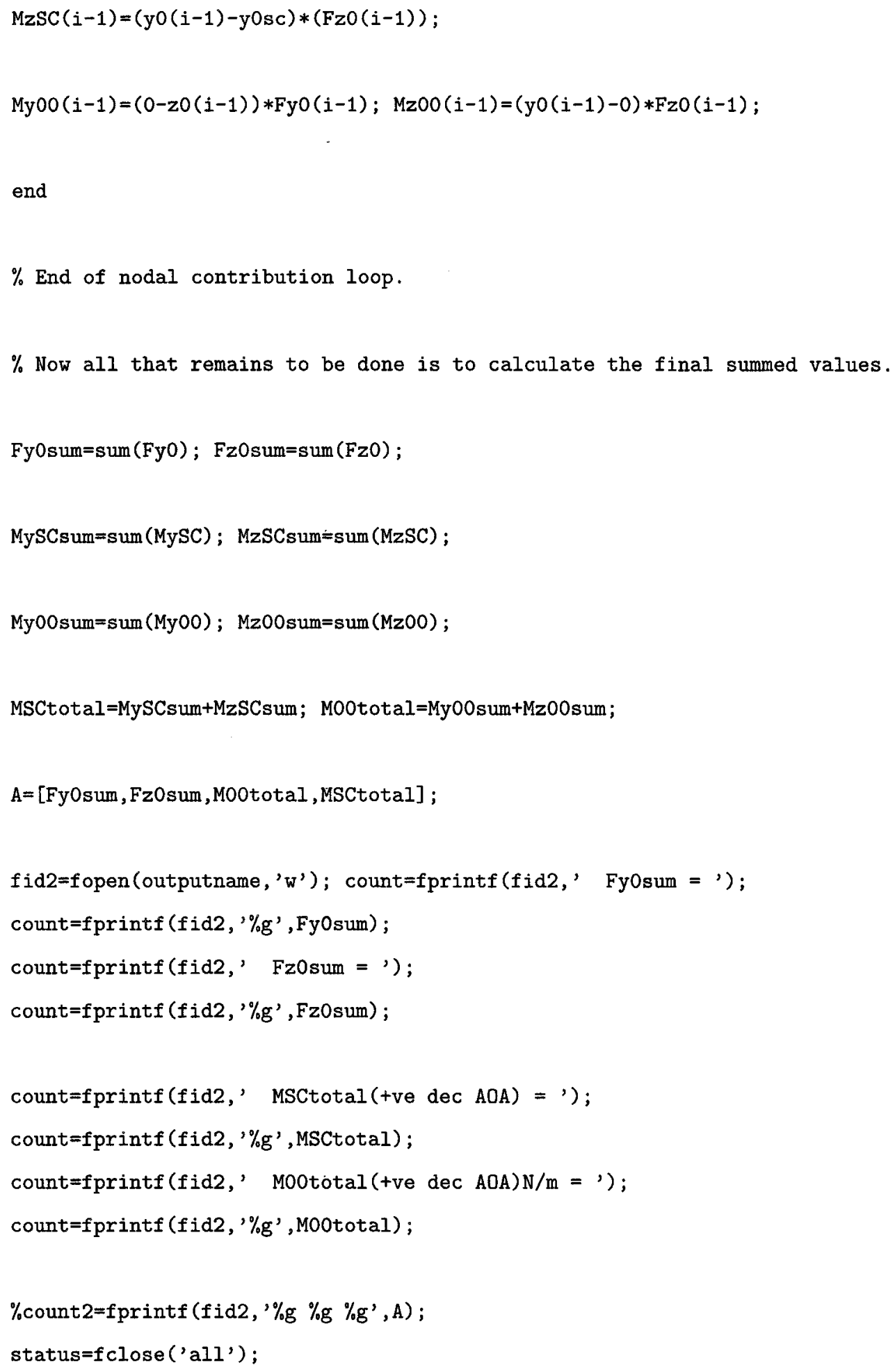




\section{Appendix B}

\section{Sample Matlab input data: pressure data}

\section{for mode 1 at timestep 6501}

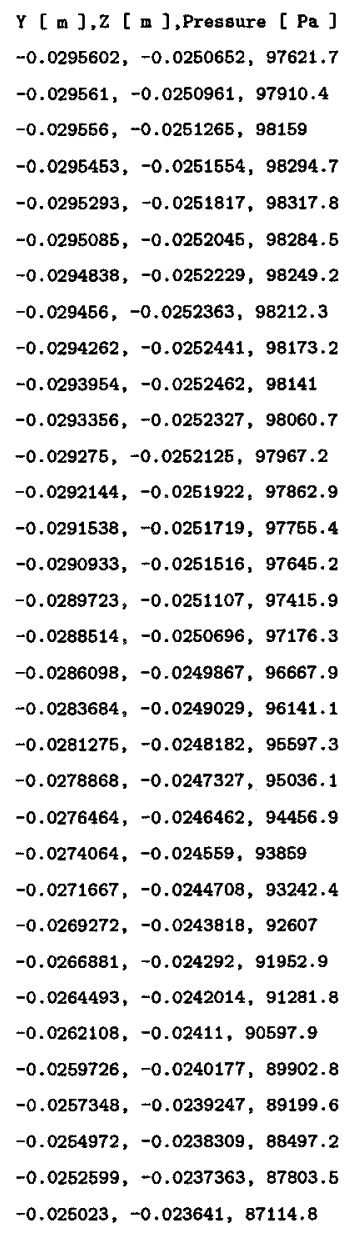

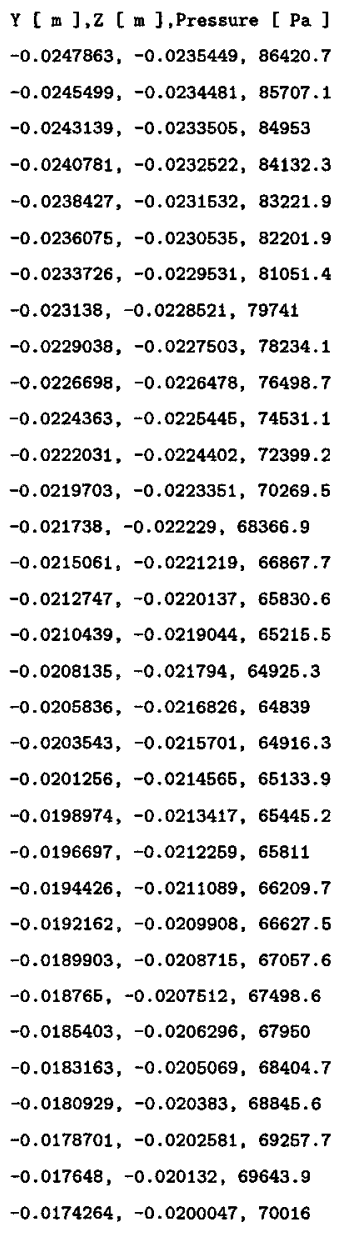

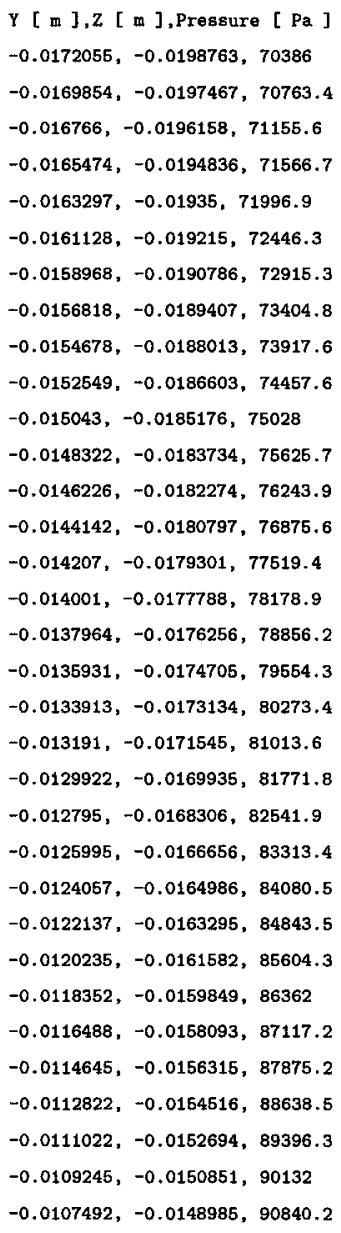


$\mathrm{Y}[\mathrm{m}], \mathrm{Z}[\mathrm{m}]$, Pressure [ Pa ] $-0.0105765,-0.0147097,91532.2$ $-0.0104063,-0.0145186,92218.8$ $-0.0102387,-0.0143253,92894.1$ $-0.0100739,-0.0141297,93531.7$ $-0.00991192,-0.0139318,94108.2$ $-0.00975273,-0.0137317,94627.9$ $-0.00959656,-0.0135292,96107.5$ $-0.00944359,-0.0133244,95561.8$ $-0.009294,-0.0131171,95990.7$ $-0.00914798,-0.0129074,96385.4$ $-0.00900571,-0.0126951,96745.3$ $-0.00886738,-0.0124802,97088.3$ $-0.00873324,-0.0122626,97447.6$ $-0.00860362,-0.0120421,97840.5$ $-0.00847876,-0.0118188,98267.4$ $-0.00836893,-0.0115926,98728.5$ $-0.00824436,-0.0113632,99238.6$ $-0.00813532,-0.0111307,99834.6$ $-0.00803205,-0.010895,100536$ $-0.00793481,-0.010666,101335$ $-0.00784385,-0.0104135,102216$ $-0.00775924,-0.010168,103146$ $-0.0076809,-0.00991985,104078$ $-0.00760882,-0.00966965,104979$ $-0.00754291,-0.00941791,105821$ $-0.00748315,-0.00916515,106589$ $-0.00742948,-0.00891188,107276$ $-0.00738184,-0.00865864,107872$ $-0.00734019,-0.00840593,108348$ $-0.00730447,-0.0081543,108663$ $-0.00727462,-0.00790427,108759$ $-0.0072506,-0.00765636,108709$ $-0.00723216,-0.00741139,108429$ $-0.00721985,-0.00716897,108033$ $-0.00721484,-0.00692785,107706$ $-0.00721829,-0.00668674,107586$ $-0.00723151,-0.00644413,107601$ $-0.00725699,-0.00619832,107956$ $-0.00729308,-0.0059477,108723$ $-0.00734417,-0.00569069,109859$ $-0.00741063,-0.00642571,111218$ $-0.00749383,-0.00515118,112491$ $-0.0075952,-0.0048652,113361$ $-0.00771483,-0.00457334,113662$ $-0.00786177,-0.00428679,113369$ $-0.00800469,-0.00401905,112517$ $-0.00817231,-0.00378318,110853$ $-0.00835335,-0.00359226,109967$ $-0.00854654,-0.00346937,115700$ $-0.00875062,-0.00339757,126827$ $-0.00896426,-0.00341981,141341$ $-0.0091824,-0.00352621,147563$
Y [ [ ] ], Z [ m ],Pressure [ Pa ] $-0.00939298,-0.00369318,149739$ $-0.0095833,-0.00389466,150101$ $-0.00974398,-0.00410981,149893$ $-0.00987639,-0.00433436,149366$ $-0.00998397,-0.00456718,148799$ $-0.0100701,-0.00480716,148347$ $-0.0101382,-0.00505318,148002$ $-0.0101918,-0.0053041,147799$ $-0.0102341,-0.00555882,147714$ $-0.0102686,-0.00581621,147701$ $-0.0102988,-0.00607515,147713$ $-0.0103281,-0.00633452,147758$ $-0.0103598,-0.0065932,147817$ $-0.0103974,-0.00685007,147863$ $-0.0104436,-0.00710423,147886$ $-0.0104984,-0.00735563,147890$ $-0.0105613,-0.00760439,147882$ $-0.0106317,-0.00785066,147853$ $-0.0107092,-0.00809455,147808$ $-0.0107931,-0.00833621,147761$ $-0.0108829,-0.00867576,147687$ $-0.0109781,-0.00881334,147614$ $-0.0110782,-0.00904907,147636$ $-0.0111825,-0.00928308,147454$ $-0.0112906,-0.00951554,147368$ $-0.0114018,-0.00974655,147282$ $-0.0115158,-0.0099762,147198$ $-0.0116321,-0.0102046,147118$ $-0.0117507,-0.0104317,147044$ $-0.0118716,-0.0106574,146976$ $-0.011995,-0.0108817,146910$ $-0.0121209,-0.0111044,146845$ $-0.0122495,-0.0113254,146780$ $-0.0123807,-0.0115448,146716$ $-0.0125147,-0.0117623,146651$ $-0.0126515,-0.011978,146584$ $-0.0127913,-0.0121916,146515$ $-0.012934,-0.0124033,146443$ $-0.0130798,-0.0126128,146365$ $-0.0132288,-0.0128201,146282$ $-0.0133808,-0.0130252,146190$ $-0.0135359,-0.013228,146090$ $-0.0136939,-0.0134288,145982$ $-0.0138546,-0.0136274,145867$ $-0.014018,-0.013824,145746$ $-0.0141837,-0.0140187,145618$ $-0.0143518,-0.0142114,146484$ $-0.0145221,-0.0144023,145344$ $-0.0146944,-0.0145914,145198$ $-0.0148686,-0.0147788,145046$ $-0.0150445,-0.0149645,144890$ $-0.0152221,-0.0151486,144730$
Y [ m ],Z [ m ],Pressure [ $\mathrm{Pa}]$ $-0.0154012,-0.0153311,144566$ $-0.0155819,-0.0155121,144396$ $-0.0157639,-0.0156915,144222$ $-0.0159472,-0.0158695,144046$ $-0.0161318,-0.0160461,143867$ $-0.0163177,-0.0162213,143685$ $-0.0165048,-0.0163952,143498$ $-0.0166932,-0.0165677,143306$ $-0.0168829,-0.0167388,143109$ $-0.0170737,-0.0169086,142906$ $-0.0172657,-0.017077,142699$ $-0.0174589,-0.0172441,142486$ $-0.0176532,-0.0174098,142266$ $-0.0178486,-0.0175743,142039$ $-0.0180462,-0.0177374,141806$ $-0.0182428,-0.0178993,141566$ $-0.0184415,-0.0180598,141317$ $-0.0186412,-0.0182191,141060$ $-0.018842,-0.018377,140795$ $-0.0190437,-0.0185337,140521$ $-0.0192464,-0.0186891,140236$ $-0.0194501,-0.0188433,139941$ $-0.0196546,-0.0189963,139637$ $-0.0198601,-0.0191481,139324$ $-0.0200663,-0.0192987,138999$ $-0.0202734,-0.0194482,138664$ $-0.0204814,-0.0195966,138318$ $-0.0206901,-0.0197439,137961$ $-0.0208995,-0.0198901,137593$ $-0.0211097,-0.0200363,137214$ $-0.0213205,-0.0201795,136823$ $-0.0215321,-0.0203227,136420$ $-0.0217443,-0.0204649,136004$ $-0.0219571,-0.0206062,135676$ $-0.0221705,-0.0207465,135137$ $-0.0223845,-0.020886,134689$ $-0.0225991,-0.0210245,134229$ $-0.0228143,-0.0211622,133753$ $-0.02303,-0.0212989,133259$ $-0.0232463,-0.0214348,132748$ $-0.0234631,-0.0216698,132222$ $-0.0236804,-0.021704,131679$ $-0.0238982,-0.0218373,131120$ $-0.0241166,-0.0219699,130544$ $-0.0243353,-0.0221017,129951$ $-0.0245546,-0.0222328,129337$ $-0.0247742,-0.0223631,128702$ $-0.0249943,-0.0224928,128047$ $-0.0252148,-0.0226217,127373$ $-0.0254357,-0.0227501,126681$ $-0.0256569,-0.0228777,125969$ $-0.0258785,-0.0230048,125240$ 
$\mathrm{Y}[\mathrm{m}], \mathrm{Z}[\mathrm{m}]$, Pressure $[\mathrm{Pa}]$ $-0.0261005,-0.0231312,124494$ $-0.0263228,-0.0232569,123723$ $-0.0265466,-0.0233819,122918$ $-0.0267689,-0.023606,122067$ $-0.0269925,-0.0236293,121163$ $-0.0272167,-0.0237518,120194$ $-0.0274413,-0.0238733,119146$ $-0.0276665,-0.0239939,117993$ $-0.0278922,-0.0241135,116695$ $-0.0281183,-0.0242322,115211$ $-0.0283446,-0.0243506,113519$ $-0.0285712,-0.0244685,111599$ $-0.0287977,-0.0245864,109389$ $-0.0290241,-0.0247046,106764$ $-0.0291372,-0.0247639,104987$ $-0.0292503,-0.0248234,102681$ $-0.0293067,-0.0248532,101101$ $-0.0293632,-0.024883,99088$ $-0.0294196,-0.0249129,96478.9$ $-0.029476,-0.0249429,93136.2$

$-0.0295237,-0.0249815,91114.6$ $-0.0295412,-0.0250069,95043.8$ $-0.0295535,-0.0260351,97247.7$ 


\section{Appendix C}

\section{Matlab m-file output for sample input}

The postprocessing $\mathrm{m}$-file is executed with the following command.

[FyOsum, FzOsum, MO0total, MSCtotal, dadd] =PostProcessing ('input.txt' , 'output . out')

The output acquired for timestep 6501 in the data set corresponding to the first natural mode shape is as follows.

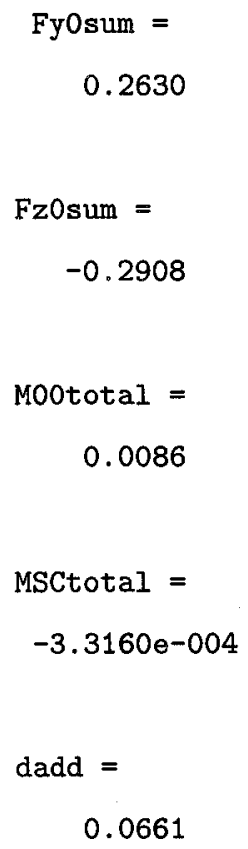




\section{Appendix D}

\section{Curve fit plots}

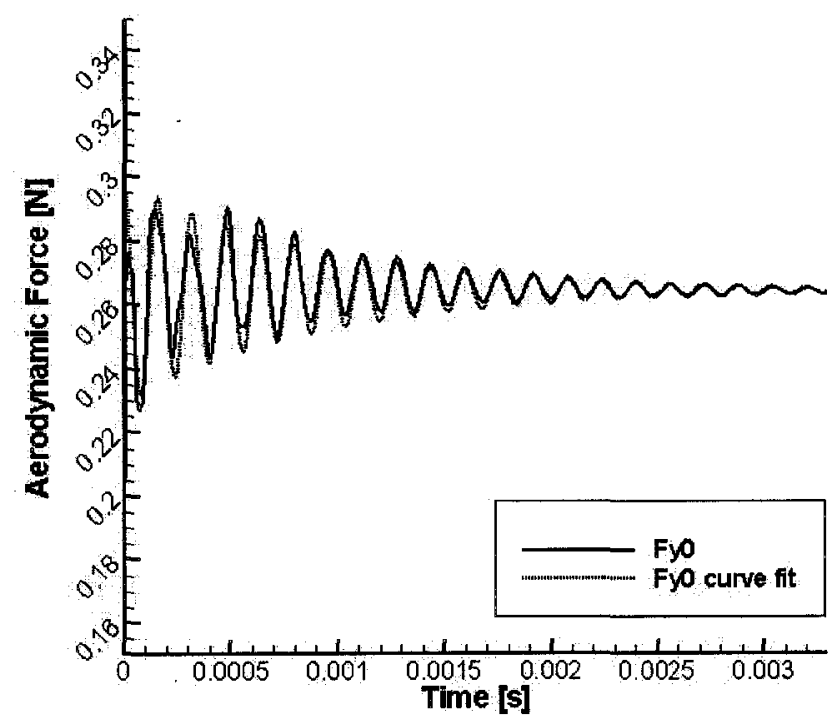

Figure D.1: Mode 1 - force in the $y_{0}$ direction over time 


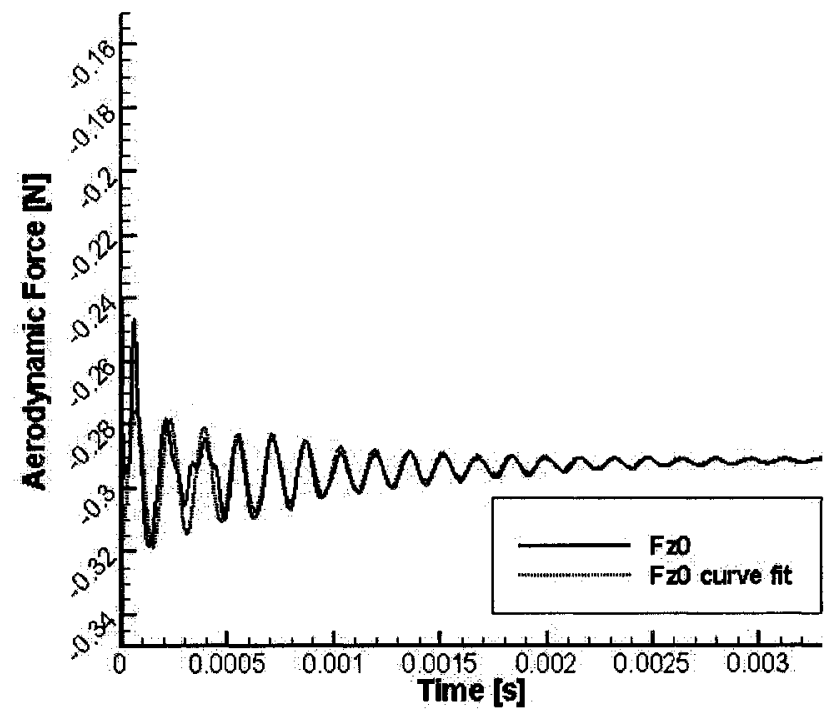

Figure D.2: Mode 1 - force in the $z_{0}$ direction over time

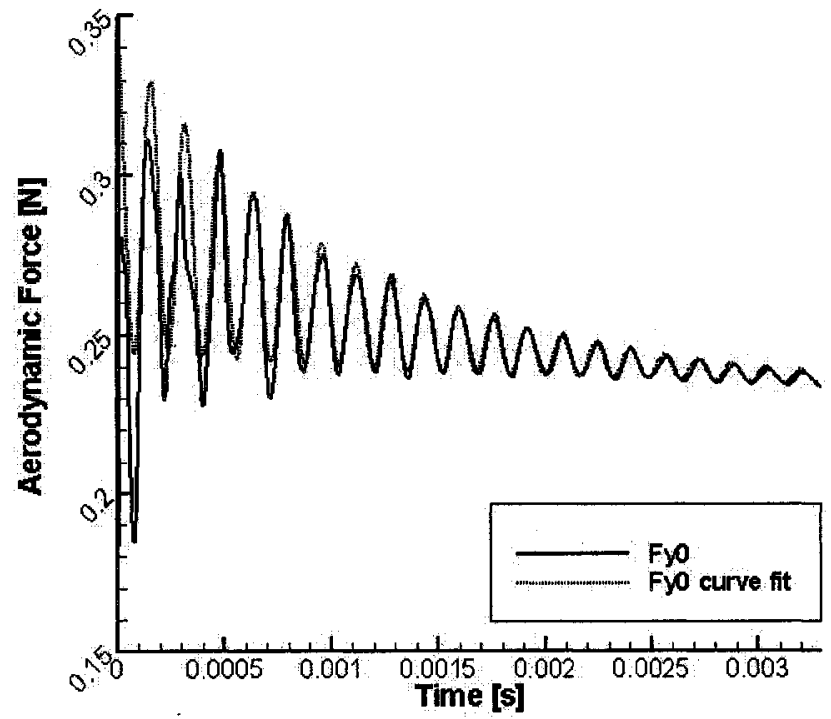

Figure D.3: Mode 2 - force in the $y_{0}$ direction over time 


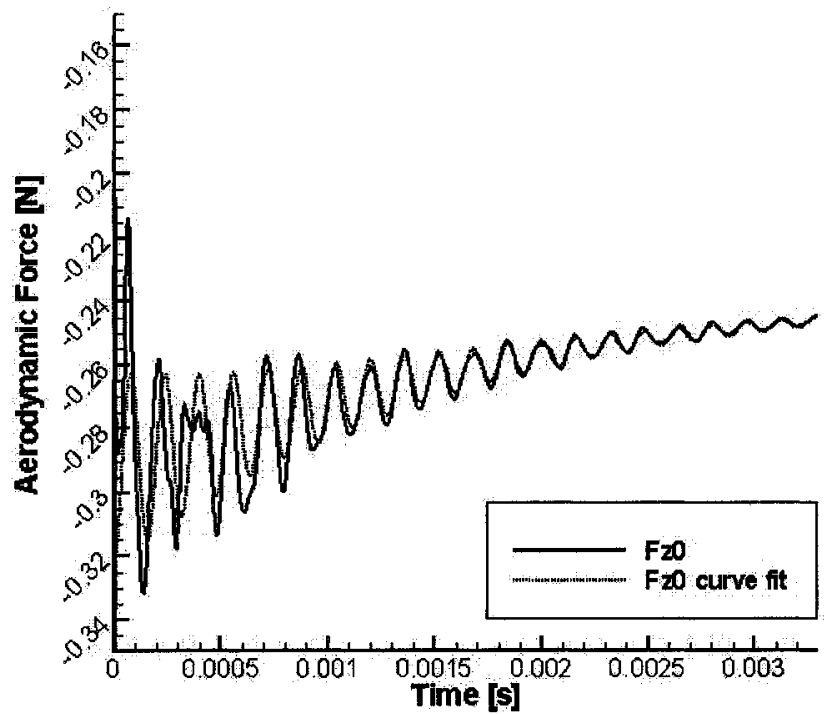

Figure D.4: Mode 2 - force in the $z_{0}$ direction over time

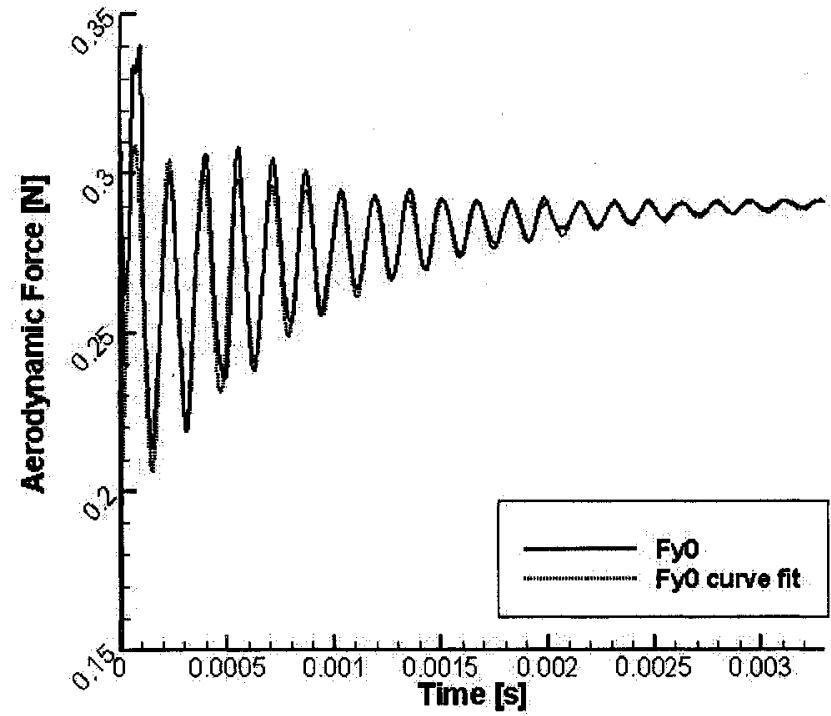

Figure D.5: Mode 3 - force in the $y_{0}$ direction over time 


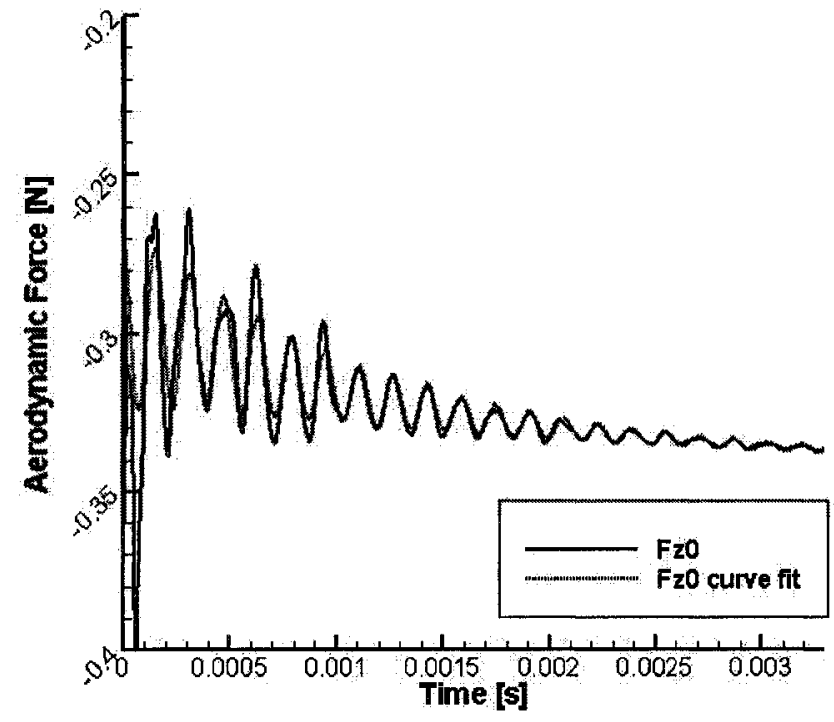

Figure D.6: Mode 3 - force in the $z_{0}$ direction over time 


\section{Appendix E}

\section{Nodal matrix calculated by ANSYS}

[u] -

$\begin{array}{rrr}0 & 0 & 0 \\ -3.8038 & 1.4769 & 6.5706 \\ -11.9210 & 5.2957 & 15.5120 \\ -24.2560 & 11.1770 & 29.2910 \\ -39.6690 & 18.7490 & 44.9840 \\ -67.6990 & 27.3090 & 61.8640 \\ -77.8820 & 36.6470 & 79.1540 \\ -99.7600 & 46.5750 & 96.2390 \\ -122.9100 & 56.9220 & 112.6800 \\ -146.9200 & 67.5430 & 128.2300 \\ -169.4900 & 75.9670 & 142.1100 \\ -194.3500 & 86.7690 & 155.8600 \\ -222.3200 & 104.4500 & 168.7300 \\ 0 & 0 & 0 \\ 3.7509 & 4.8178 & 1.4399 \\ 11.8310 & 11.8980 & 5.2365 \\ 24.0620 & 20.0140 & 12.5080 \\ 39.2710 & 27.3740 & 23.6590 \\ 56.9960 & 33.9360 & 38.7490 \\ 76.7800 & 39.4570 & 57.7400 \\ 98.1770 & 43.7560 & 80.3590 \\ 120.7700 & 46.6910 & 106.1300 \\ 144.1700 & 48.1480 & 134.4000 \\ 166.1000 & 49.3760 & 161.4500 \\ 190.2300 & 47.9630 & 192.3900 \\ 217.3100 & 40.1860 & 228.9900\end{array}$

